\title{
Palladium-Catalyzed Regio- and Diastereoselective Olefinic C-H Difluoromethylthiolation at Room
}

Temperature

Tongxu Xiang, ${ }^{1}$ Yongze Liu, ${ }^{1}$ Qiaoling Xu, ${ }^{1}$ Kehan $\mathrm{He},{ }^{* 2}$ and Fei Pan ${ }^{\star 1}$

${ }^{1}$ College of Chemistry and Materials Science, Sichuan Normal University, 5 Jingan Road, Chengdu 610068, P. R. China

${ }^{2}$ School of Science, Xichang University, 1 Xuefu Road, Xichang 615000, P. R. China *Email: hdss2006@163.com; feipan@sicnu.edu.cn.

1. Mechanistic Study... S02

2. NMR Spectra. S06 


\section{Mechanistic Study}

1.1 Radical trapping experiments

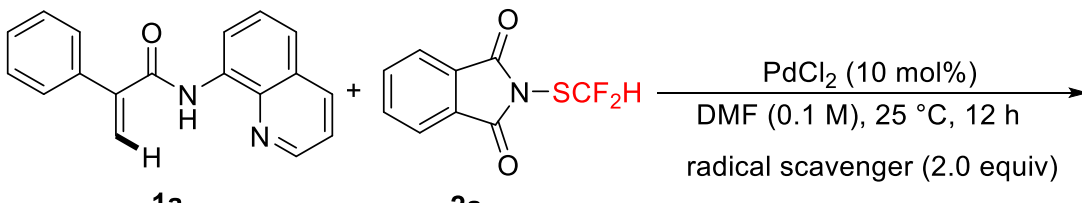

$1 \mathrm{a}$

2a

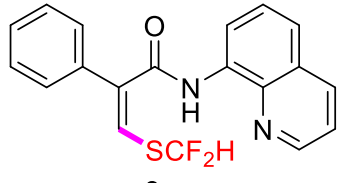

3a

with TEMPO: $88 \%$ with BHT: $90 \%$

A dried tube was added $\mathrm{PdCl}_{2}$ (3.5 $\mathrm{mg}, 0.02 \mathrm{mmol}, 0.1$ equiv), 2-phenyl- $\mathrm{N}$-(quinolin-8yl)acrylamide 1a (54.8 mg, $0.2 \mathrm{mmol}, 1.0$ equiv), Phth-SCF${ }_{2} \mathrm{H}$ 2a (91.6 mg, $0.4 \mathrm{mmol}$, 2.0 equiv), and radical scavenger ( $0.4 \mathrm{mmol}, 2.0$ equiv). Then DMF ( $2 \mathrm{~mL}, 0.1 \mathrm{M})$ was injected. The tube was sealed with a rubber septum and the suspension was stirred at $25{ }^{\circ} \mathrm{C}$ for $12 \mathrm{~h}$. Then we used GC-MS to monitor the reaction mixture, and no radical intermediated was observed. The reaction mixture was quenched by the addition of 20 $\mathrm{mL}$ water, and extracted with dichloromethane $(3 \times 15 \mathrm{~mL})$. The combined organic layer was washed with brine $(2 \times 30 \mathrm{~mL})$, dried over anhydrous $\mathrm{MgSO}_{4}$, and concentrated in vacuum. The residue was purified by column chromatography on silica gel to afford the product 3a (with TEMPO: $62.6 \mathrm{mg}, 88 \%$ yield; with BHT: $64.0 \mathrm{mg}, 90 \%$ yield).

1.2 Intermolecular competition experiment<smiles>C/C=C(\C(=O)Nc1cccc2cccnc12)c1ccc(F)cc1</smiles>

1c, $0.2 \mathrm{mmol}$<smiles>COc1ccc(/C=C\C(=O)Nc2cccc3cccnc23)cc1</smiles>

$1 \mathrm{j}, 0.2 \mathrm{mmol}$<smiles>O=C(Nc1cccc2cccnc12)/C(=C\C(F)F)c1ccc(F)cc1</smiles>

$3 c, 33 \%$<smiles>COc1ccc(/C(=C/[S-]F)C(=O)Nc2cccc3cccnc23)cc1</smiles>

$3 \mathbf{j}, 42 \%$

A dried tube was added $\mathrm{PdCl}_{2}$ (3.5 mg, $0.02 \mathrm{mmol}, 0.1$ equiv), 2-(4-fluorophenyl)-N(quinolin-8-yl)acrylamide 1c (58.4 mg, $0.2 \mathrm{mmol}, 1.0$ equiv), 2-(4-methoxyphenyl)-N(quinolin-8-yl)acrylamide $1 \mathbf{j}$ ( $60.8 \mathrm{mg}, 0.2 \mathrm{mmol}, 1.0$ equiv), and Phth-SCF ${ }_{2} \mathrm{H} \mathrm{2a}$ (45.8 $\mathrm{mg}, 0.4 \mathrm{mmol}, 1.0$ equiv). Then DMF ( $2 \mathrm{~mL}, 0.1 \mathrm{M})$ was injected. The tube was sealed with a rubber septum and the suspension was stirred at $25^{\circ} \mathrm{C}$ for $12 \mathrm{~h}$. The reaction mixture was quenched by the addition of $20 \mathrm{~mL}$ water, and extracted with dichloromethane $(3 \times 15 \mathrm{~mL})$. The combined organic layer was washed with brine (2 $\times 30 \mathrm{~mL}$ ), dried over anhydrous $\mathrm{MgSO}_{4}$, and concentrated in vacuum. The residue was purified by column chromatography on silica gel to afford the products $3 \mathrm{c}$ ( $24.6 \mathrm{mg}$, $33 \%$ yield) and $\mathbf{3 j}$ (32.4 $\mathrm{mg}, 42 \%$ yield). 
1.3 Deuterium-Labeled Experiments<smiles>C/C=C(\C(=O)Nc1cccc2cccnc12)c1ccccc1</smiles>

1a

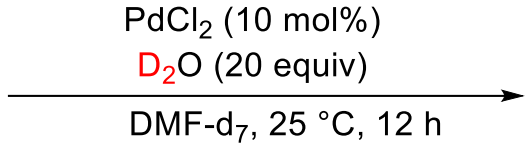

$\mathrm{PdCl}_{2}(10 \mathrm{~mol} \%)$

$2 a$ (1.0 equiv)

DMF, $25^{\circ} \mathrm{C}, 4 \mathrm{~h}$

$\mathrm{KIE}=K_{\mathrm{H}} / K_{\mathrm{D}}=2.5$<smiles>[O]/C=C(\C(=O)Nc1cccc2cccnc12)c1ccccc1</smiles>

$1 \mathrm{a}-d, 62 \% \mathrm{D}$

A dried tube was added $\mathrm{PdCl}_{2}$ (3.5 $\mathrm{mg}, 0.02 \mathrm{mmol}, 0.1$ equiv), 2-phenyl- $\mathrm{N}$-(quinolin-8yl)acrylamide $1 \mathrm{a}$ (54.8 mg, $0.2 \mathrm{mmol}, 1.0$ equiv), and $\mathrm{D}_{2} \mathrm{O}$ (78 uL, $4.0 \mathrm{mmol}, 20.0$ equiv) Then DMF- $\mathrm{d}_{7}(2 \mathrm{~mL}, 0.1 \mathrm{M})$ was injected. The tube was sealed with a rubber septum and the suspension was stirred at $25^{\circ} \mathrm{C}$ for $12 \mathrm{~h}$. The reaction mixture was quenched by the addition of $20 \mathrm{~mL}$ water, and extracted with dichloromethane $(3 \times 15 \mathrm{~mL})$. The combined organic layer was washed with brine $(2 \times 30 \mathrm{~mL})$, dried over anhydrous $\mathrm{MgSO}_{4}$, and concentrated in vacuum. The residue was purified by column chromatography on silica gel to afford the product $1 \mathrm{a}-d(62 \% \mathrm{D})$.

1.4 Intermolecular KIE experiments<smiles>C=C(C(=O)Nc1cccc2cccnc12)c1ccccc1</smiles><smiles>[2H]C([2H])=C(C(=O)Nc1cccc2cccnc12)c1ccccc1</smiles><smiles>O=C(Nc1cccc2cccnc12)/C(=C\C(F)F)c1ccccc1</smiles>

$3 a$<smiles>[2H]C(=C(F)F)/C(=C(\C(=O)Nc1cccc2cccnc12)c1ccccc1)C(F)(F)F</smiles>

$3 a-d$

Parallel reactions: (four reactions were charged with 1a, while another four were charged with [D]-1a ) were performed for different reaction time (30 min, $60 \mathrm{~min}, 90$ min, $120 \mathrm{~min}$ ). Each dried tube was added $\mathrm{PdCl}_{2}$ ( $3.5 \mathrm{mg}, 0.02 \mathrm{mmol}, 0.1$ equiv), 2phenyl-N-(quinolin-8-yl)acrylamide $1 \mathrm{a}$ or $1 \mathrm{a}-\mathrm{d}_{2}\left(0.2 \mathrm{mmol}, 1.0\right.$ equiv), and Phth-SCF${ }_{2} \mathrm{H}$ 2a (91.6 mg, 0.4 mmol, 2.0 equiv). Then DMF or DMF- $\mathrm{d}_{7}(2 \mathrm{~mL}, 0.1 \mathrm{M})$ was injected. The tube was sealed with a rubber septum and the suspension was stirred at $25^{\circ} \mathrm{C}$ for different reaction time. The yield of each reaction was determined by ${ }^{19} \mathrm{~F}$ NMR analysis of the reaction mixture. The parallel reactions provided a KIE value: $\mathrm{K}_{H} / \mathrm{K}_{D}=20 / 8=2.5$. 

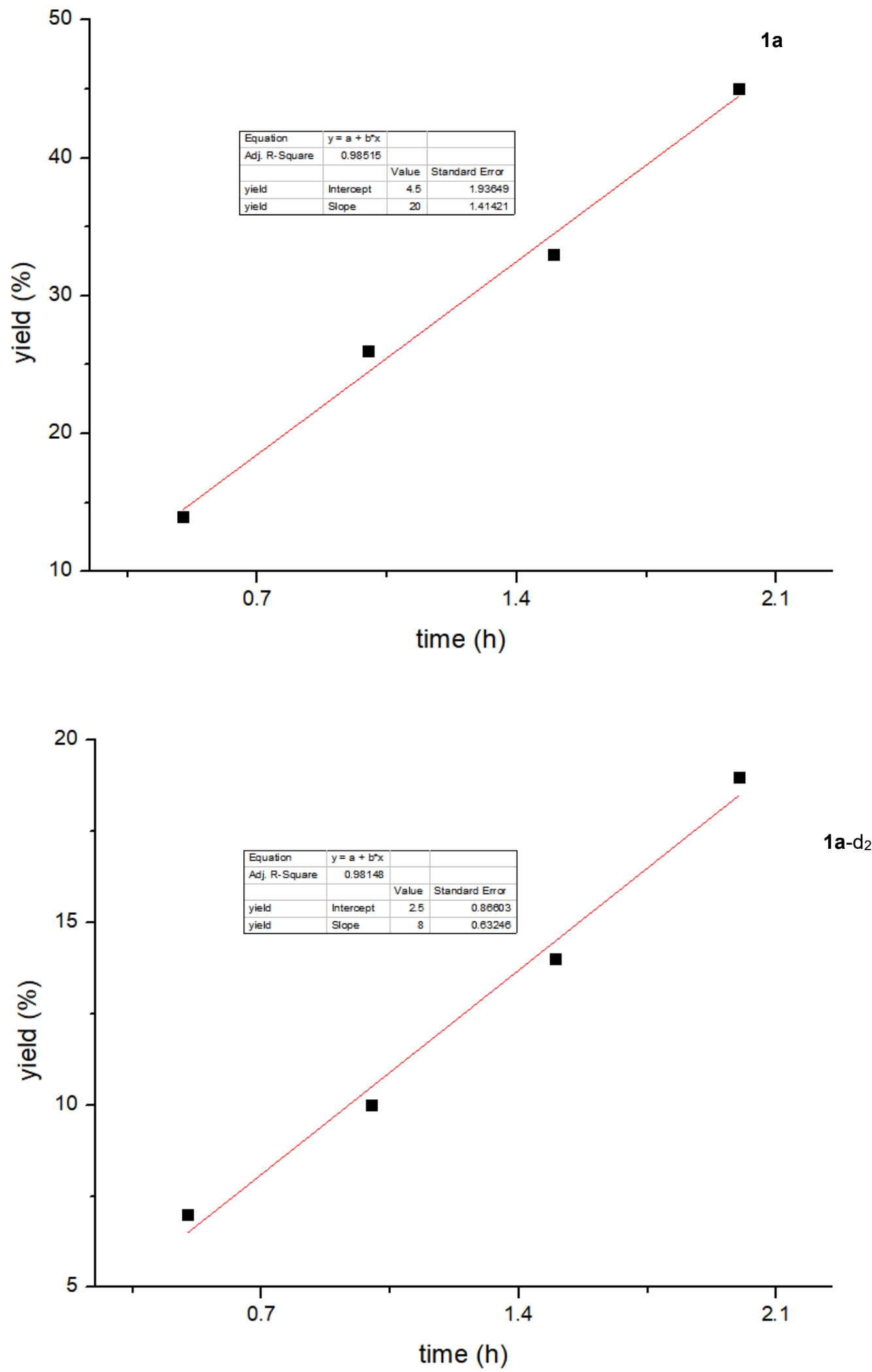

Figure S1. Kinetic experiments of $1 \mathrm{a}$ and $1 \mathrm{a}-\mathrm{d}_{2}$ 
1.5 Difluoromethylthiolation of palladacycle

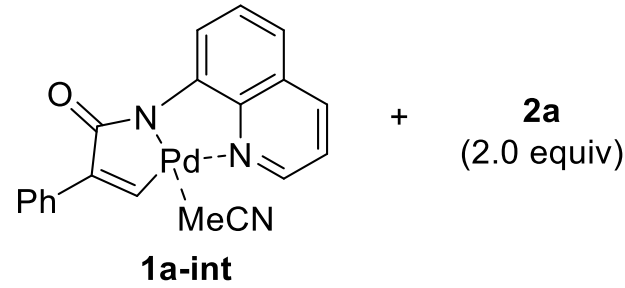<smiles></smiles>

3a, $54 \%$

A dried tube was added 1a-int (41.9 mg, $0.1 \mathrm{mmol}, 1.0$ equiv) and Phth-SCF${ }_{2} \mathrm{H} \mathrm{2a}$ (45.8 mg, $0.2 \mathrm{mmol}, 2.0$ equiv). Then DMF ( $1 \mathrm{~mL}, 0.1 \mathrm{M})$ was injected. The tube was sealed with a rubber septum and the suspension was stirred at $25^{\circ} \mathrm{C}$ for $12 \mathrm{~h}$. The reaction mixture was quenched by the addition of $20 \mathrm{~mL}$ water, and extracted with dichloromethane $(3 \times 15 \mathrm{~mL})$. The combined organic layer was washed with brine $(2$ $\times 30 \mathrm{~mL}$ ), dried over anhydrous $\mathrm{MgSO}_{4}$, and concentrated in vacuum. The residue was purified by column chromatography on silica gel to afford the product $3 \mathrm{a}(19.2 \mathrm{mg}, 54 \%$ yield).

The 2D NOESY NMR of 3a:<smiles>CCCc1ccccc1C(C(=O)Nc1cccc2cccnc12)=C(C(F)(F)F)C(F)(F)F</smiles>

$3 a$
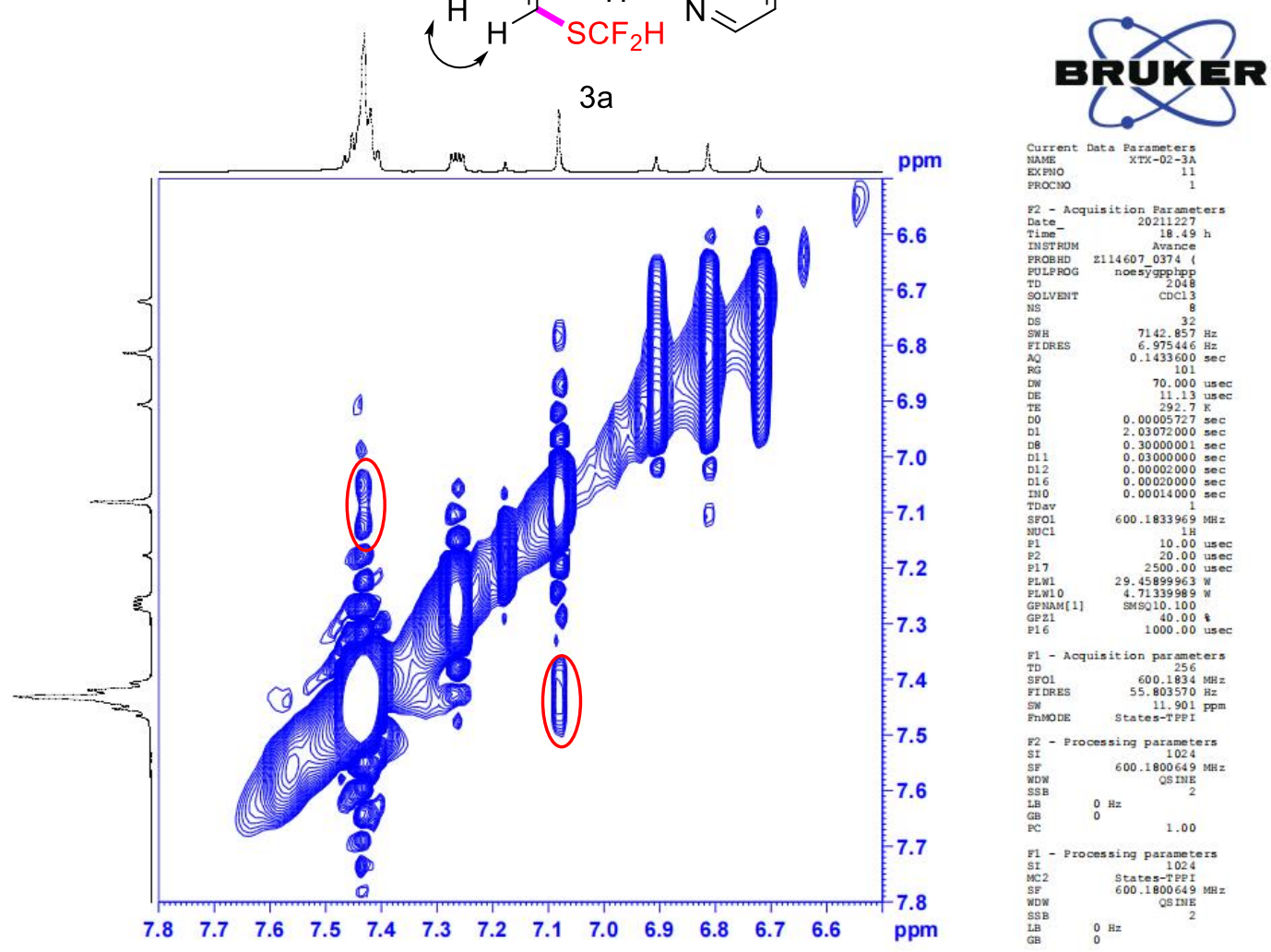


\section{NMR Spectra}

${ }^{1} \mathrm{H}$ NMR spectrum of (Z)-3-((difluoromethyl)thio)-2-phenyl-N-(quinolin-8-yl)acrylamide (3a)

$600 \mathrm{MHz}, \mathrm{CDCl}_{3}, 23{ }^{\circ} \mathrm{C}$

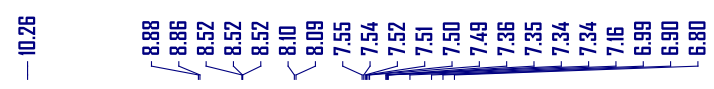<smiles>O=C(Nc1cccc2cccnc12)/C(=C\CSC(F)F)c1ccccc1</smiles>

$3 a$

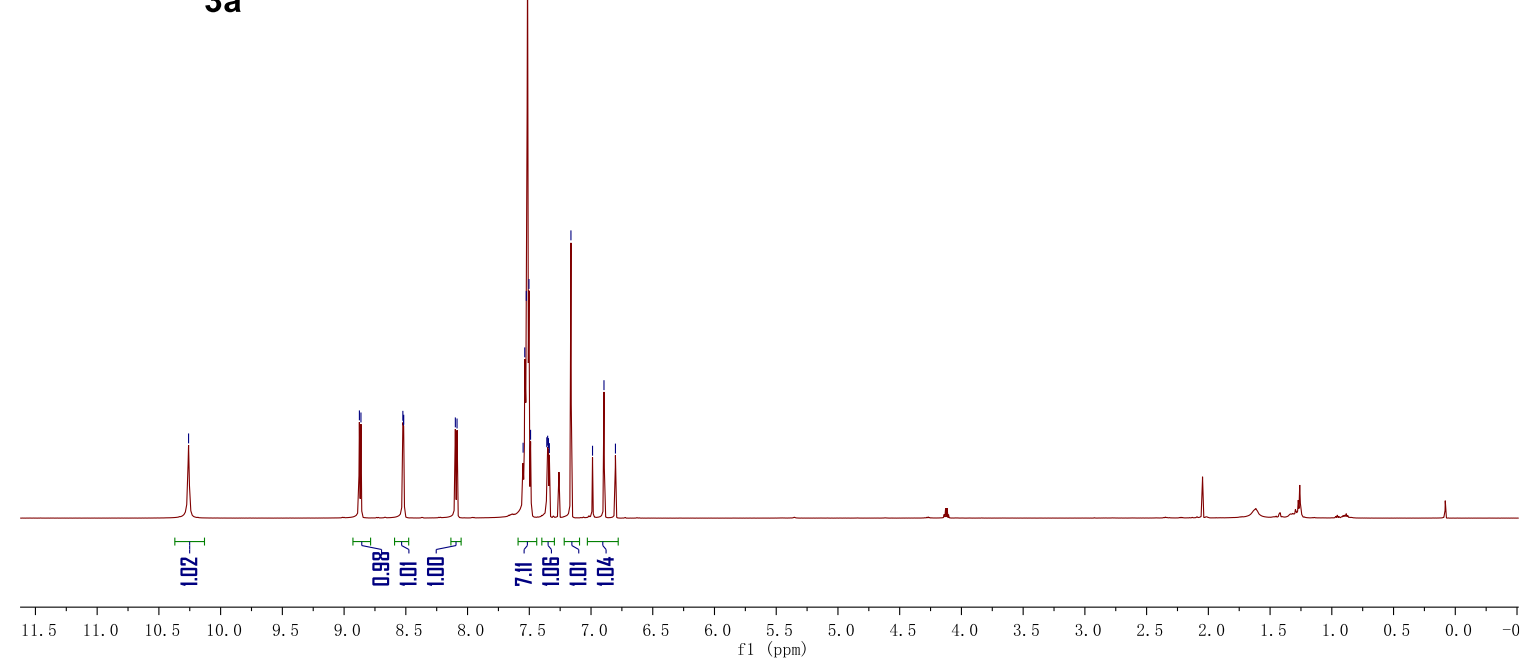

${ }^{13} \mathrm{C}\left\{{ }^{1} \mathrm{H}\right\}$ NMR spectrum of (Z)-3-((difluoromethyl)thio)-2-phenyl-N-(quinolin-8-yl)acrylamide (3a)

$151 \mathrm{MHz}, \mathrm{CDCl}_{3}, 23{ }^{\circ} \mathrm{C}$

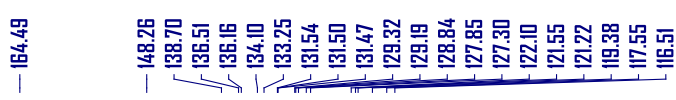<smiles>CC(F)(F)C=C(C(=O)Nc1cccc2cccnc12)c1ccccc1</smiles>

$3 a$
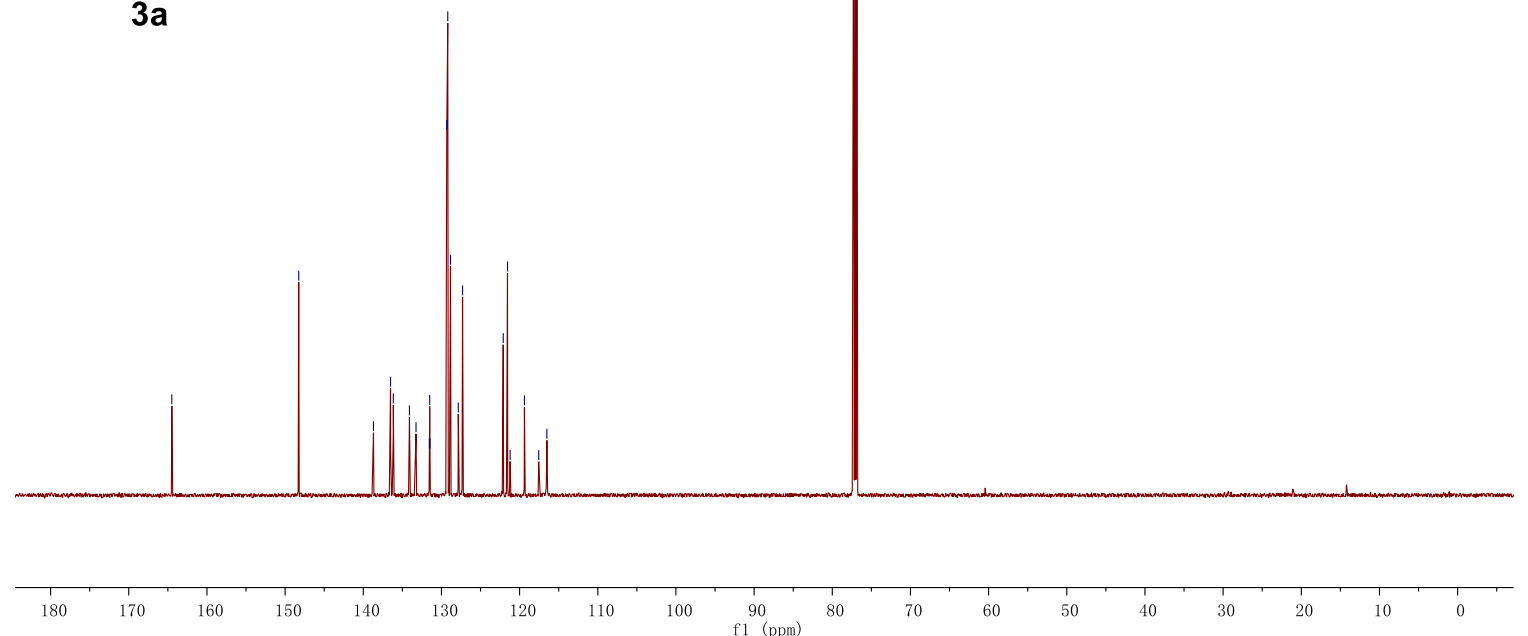
${ }^{19} \mathrm{~F}$ NMR spectrum of (Z)-3-((difluoromethyl)thio)-2-phenyl-N-(quinolin-8-yl)acrylamide (3a) $565 \mathrm{MHz}, \mathrm{CDCl}_{3}, 23{ }^{\circ} \mathrm{C}$

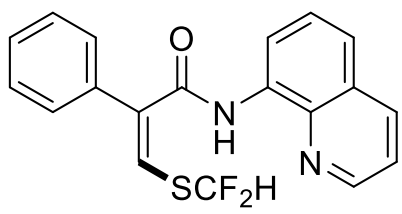

$3 a$

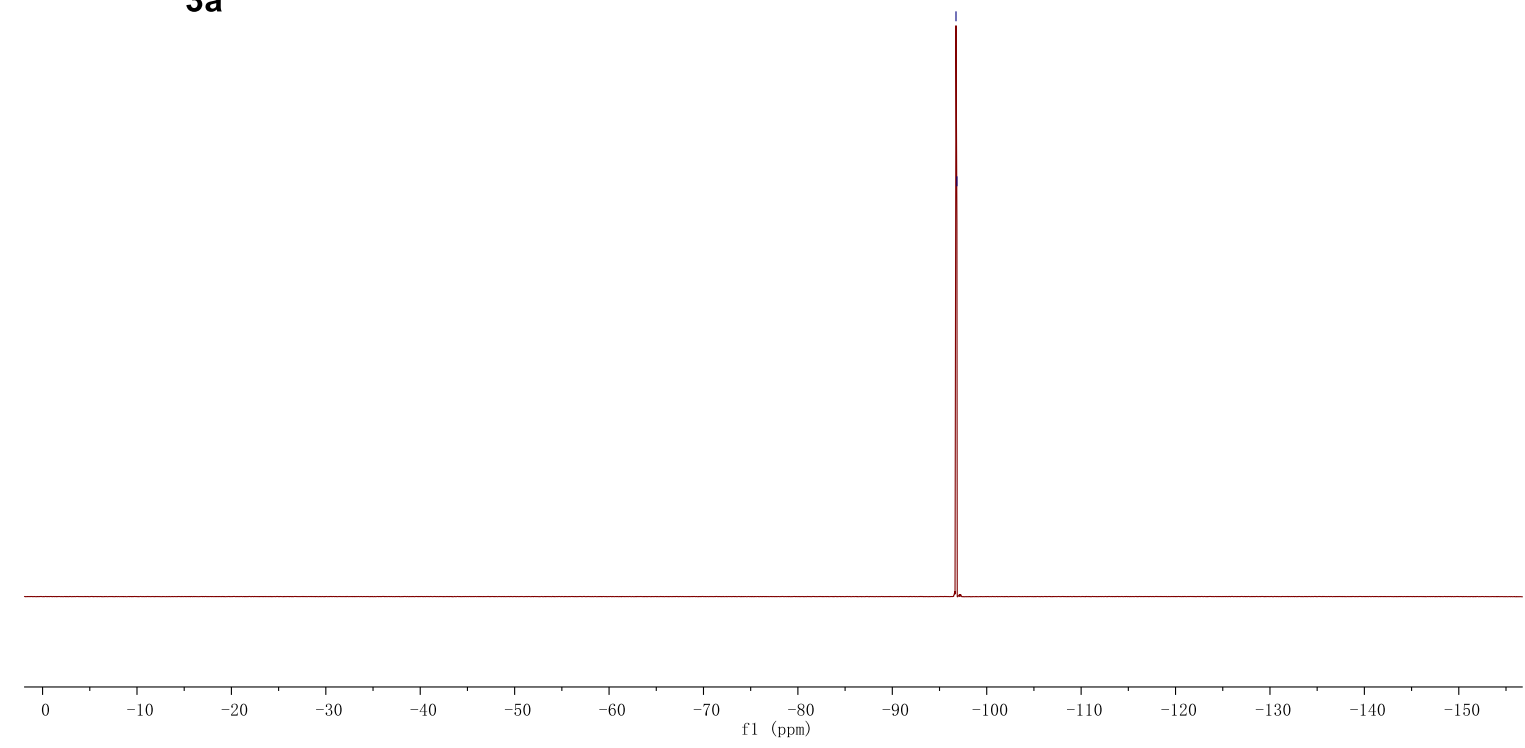

${ }^{1} \mathrm{H}$ NMR spectrum of (Z)-3-((difluoromethyl)thio)-N-(quinolin-8-yl)-2-(p-tolyl)acrylamide (3b)

$600 \mathrm{MHz}, \mathrm{CDCl}_{3}, 23{ }^{\circ} \mathrm{C}$

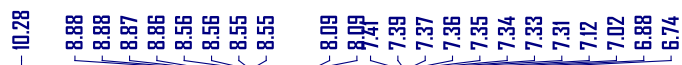<smiles>CCCCC(F)(F)F</smiles>

$3 b$

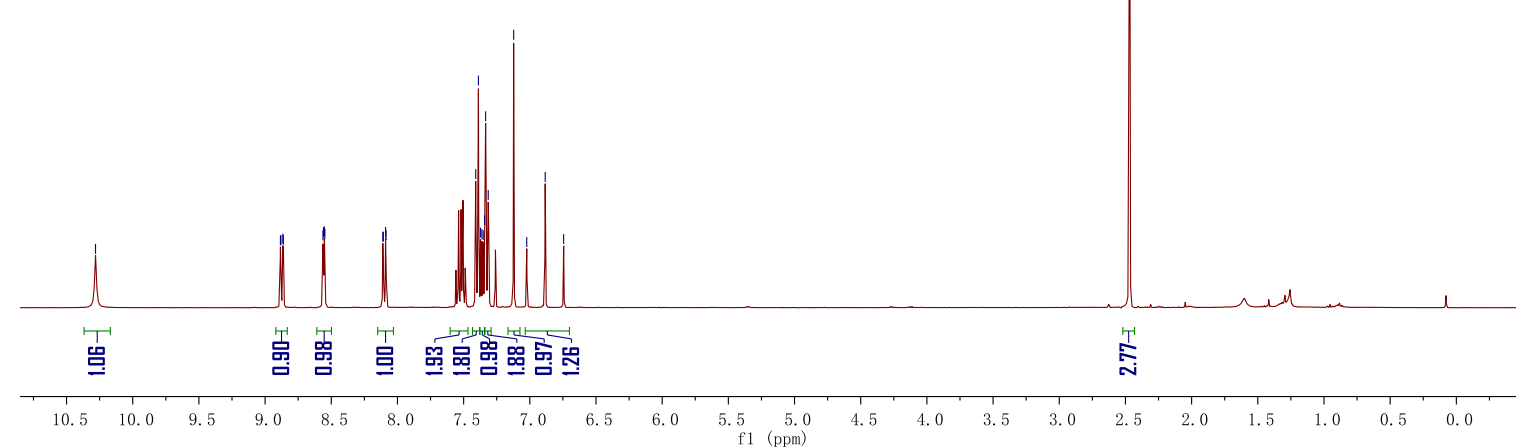


${ }^{13} \mathrm{C}\left\{{ }^{1} \mathrm{H}\right\}$ NMR spectrum of (Z)-3-((difluoromethyl)thio)-N-(quinolin-8-yl)-2-(p-tolyl)acrylamide (3b) $151 \mathrm{MHz}, \mathrm{CDCl}_{3}, 23{ }^{\circ} \mathrm{C}$

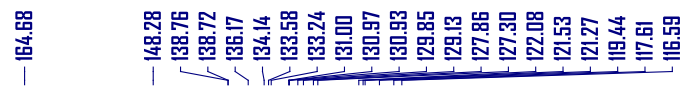<smiles>Cc1ccc(C(=CC(F)F)C(=O)Nc2cccc3cccnc23)cc1</smiles>

3b

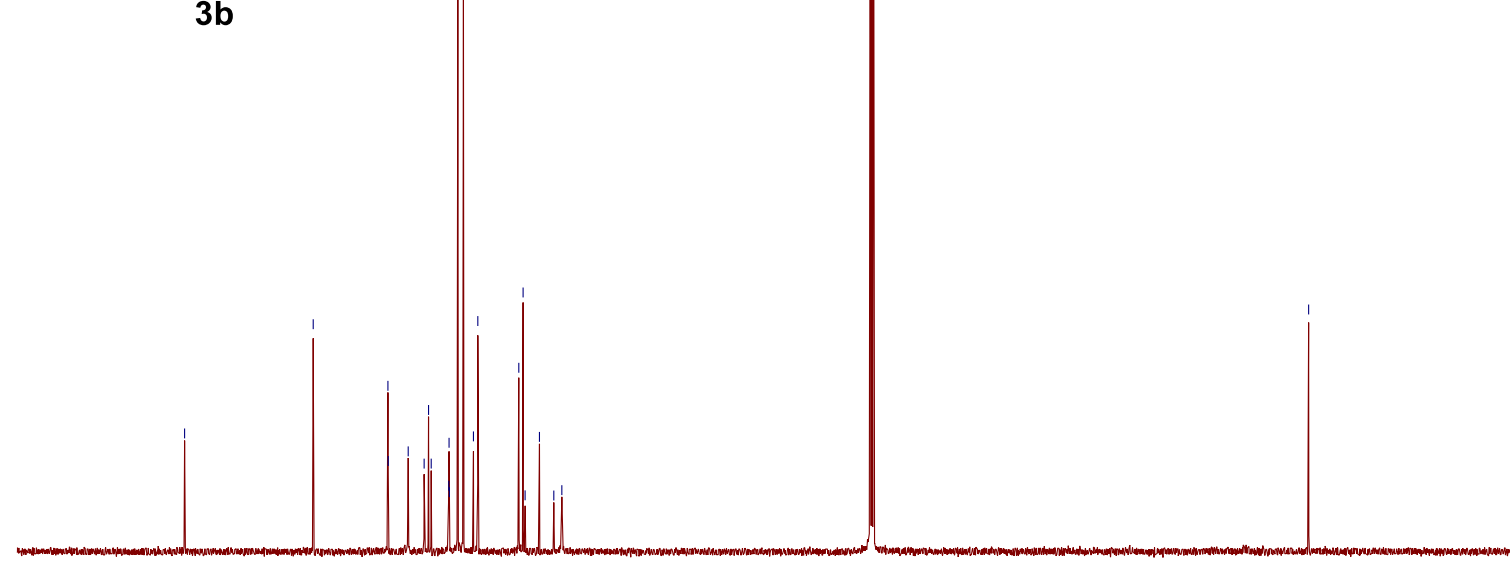

${ }^{19} \mathrm{~F}$ NMR spectrum of (Z)-3-((difluoromethyl)thio)-N-(quinolin-8-yl)-2-(p-tolyl)acrylamide (3b) $565 \mathrm{MHz}, \mathrm{CDCl}_{3}, 23{ }^{\circ} \mathrm{C}$<smiles>Cc1ccc(C(=C[SiH2]C(F)(F)F)C(=O)Nc2cccc3cccnc23)cc1</smiles>

3b

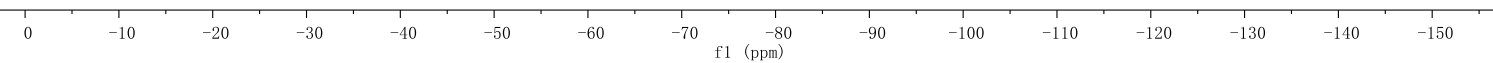


${ }^{1} \mathrm{H}$ NMR spectrum of (Z)-3-((difluoromethyl)thio)-2-(4-fluorophenyl)-N-(quinolin-8-yl)acrylamide (3c)

$600 \mathrm{MHz}, \mathrm{CDCl}_{3}, 23{ }^{\circ} \mathrm{C}$

喜<smiles>O=C(Nc1cccc2cccnc12)/C(=C\C(F)F)c1ccc(F)cc1</smiles>

3c

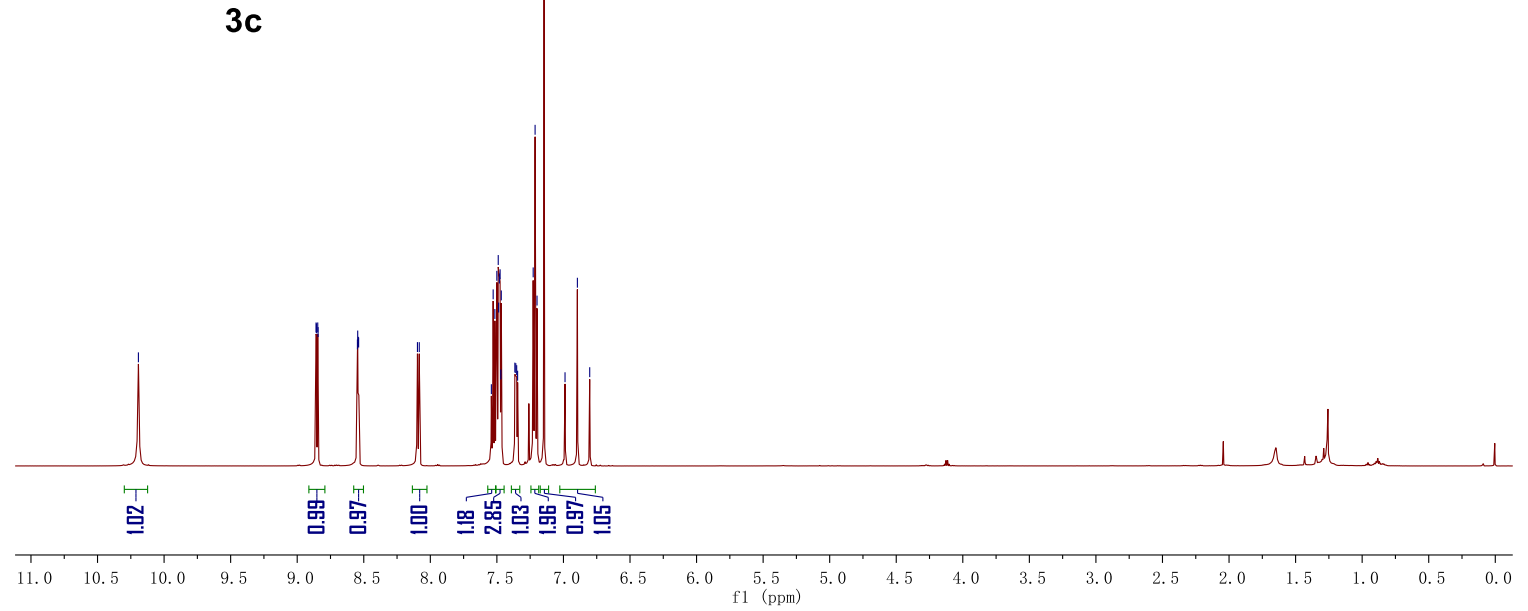

${ }^{13} \mathrm{C}\left\{{ }^{1} \mathrm{H}\right\}$ NMR spectrum of (Z)-3-((difluoromethyl)thio)-2-(4-fluorophenyl)-N-(quinolin-8-yl)acrylamide (3c) $151 \mathrm{MHz}, \mathrm{CDCl}_{3}, 23{ }^{\circ} \mathrm{C}$

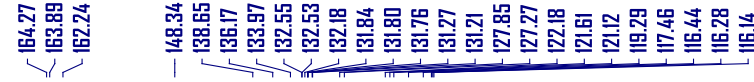<smiles>O=C(Nc1cccc2cccnc12)/C(=C\C(F)F)c1ccc(F)cc1</smiles>

$3 c$
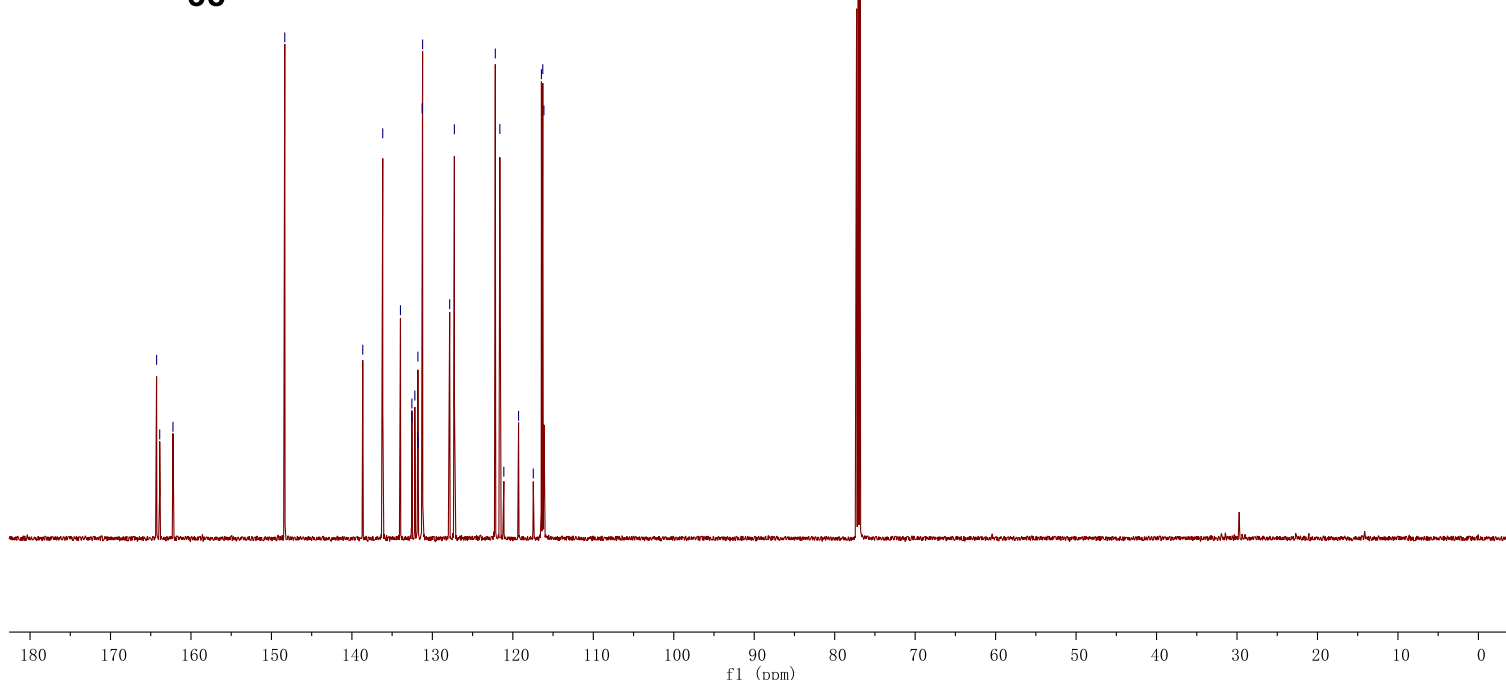
${ }^{19}$ F NMR spectrum of (Z)-3-((difluoromethyl)thio)-2-(4-fluorophenyl)-N-(quinolin-8-yl)acrylamide (3c) $565 \mathrm{MHz}, \mathrm{CDCl}_{3}, 23{ }^{\circ} \mathrm{C}$<smiles>O=C(Nc1cccc2cccnc12)/C(=C\C(F)F)c1ccc(F)cc1</smiles>

$3 c$

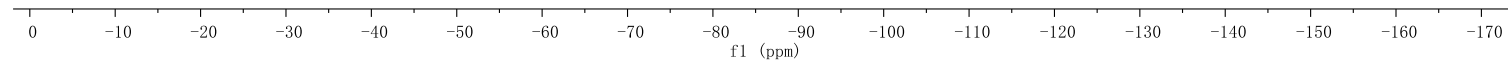

${ }^{1} \mathrm{H}$ NMR spectrum of (Z)-2-(4-chlorophenyl)-3-((difluoromethyl)thio)-N-(quinolin-8-yl)acrylamide (3d) $600 \mathrm{MHz}, \mathrm{CDCl}_{3}, 23{ }^{\circ} \mathrm{C}$

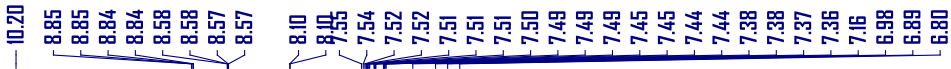<smiles>O=C(Nc1cccc2cccnc12)/C(=C\C(F)F)c1ccc(Cl)cc1</smiles>

3d

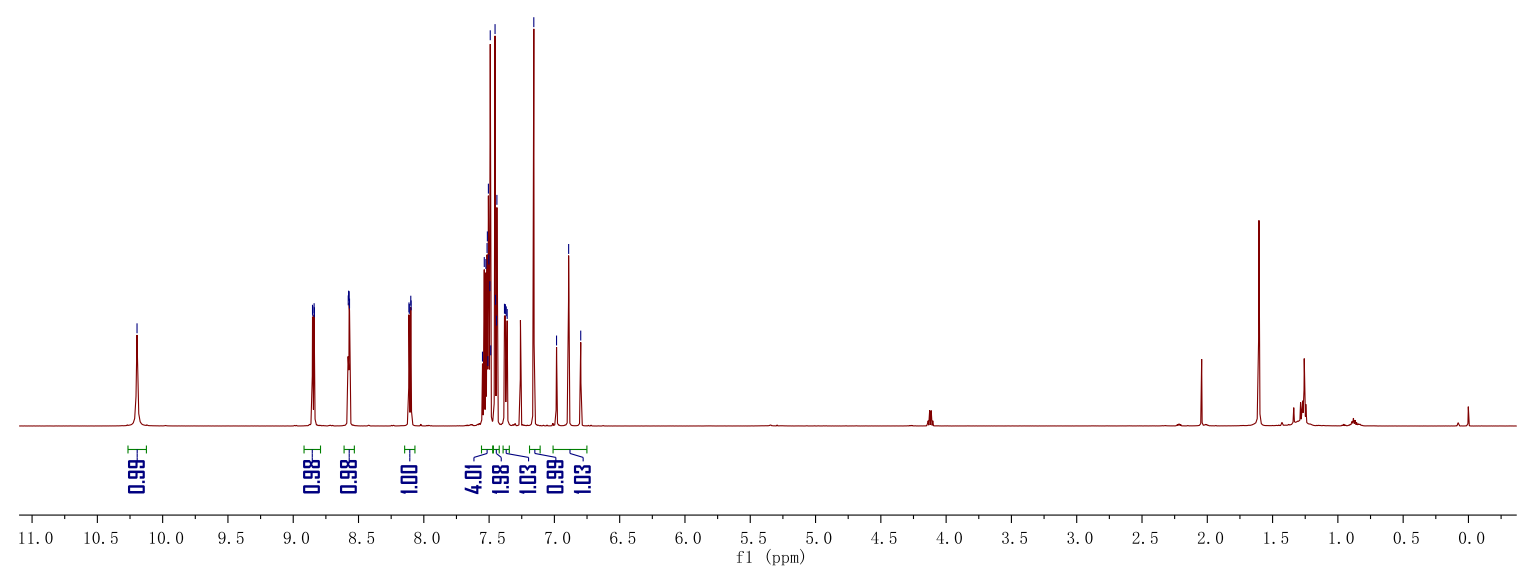


${ }^{13} \mathrm{C}\left\{{ }^{1} \mathrm{H}\right\}$ NMR spectrum of (Z)-2-(4-chlorophenyl)-3-((difluoromethyl)thio)-N-(quinolin-8-yl)acrylamide (3d) $151 \mathrm{MHz}, \mathrm{CDCl}_{3}, 23{ }^{\circ} \mathrm{C}$

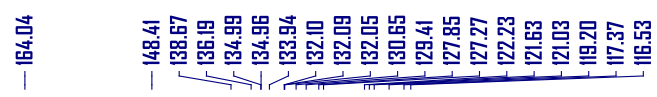<smiles>O=C(Nc1cccc2cccnc12)/C(=C\C(F)F)c1ccc(Cl)cc1</smiles>

$3 d$

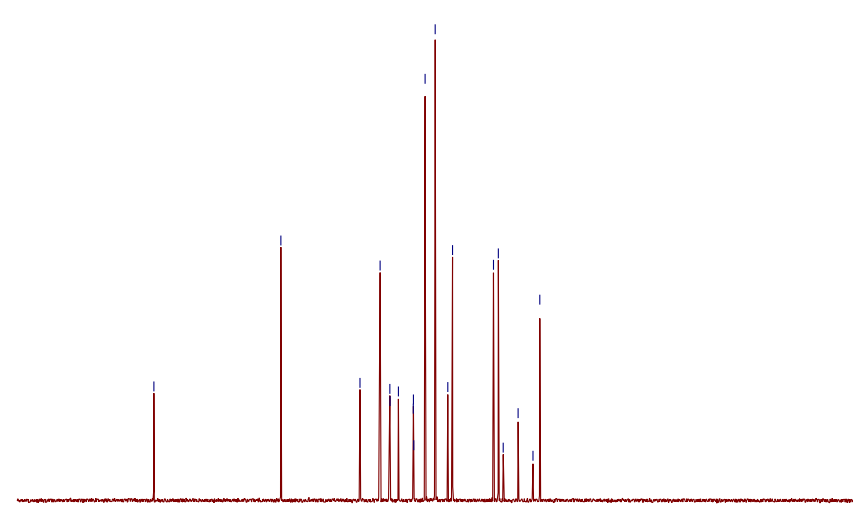

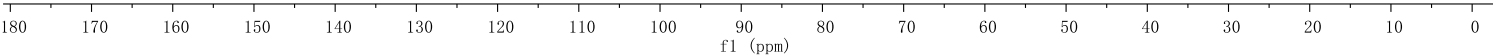

${ }^{19} \mathrm{~F}$ NMR spectrum of (Z)-2-(4-chlorophenyl)-3-((difluoromethyl)thio)- $N$-(quinolin-8-yl)acrylamide (3d) $565 \mathrm{MHz}, \mathrm{CDCl}_{3}, 23{ }^{\circ} \mathrm{C}$<smiles>O=C(Nc1cccc2cccnc12)/C(=C\C(F)F)c1ccc(Cl)cc1</smiles>

$3 d$

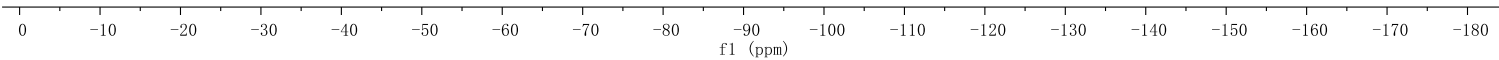


${ }^{1} \mathrm{H}$ NMR spectrum of (Z)-2-(4-bromophenyl)-3-((difluoromethyl)thio)-N-(quinolin-8-yl)acrylamide (3e)

$600 \mathrm{MHz}, \mathrm{CDCl}_{3}, 23{ }^{\circ} \mathrm{C}$

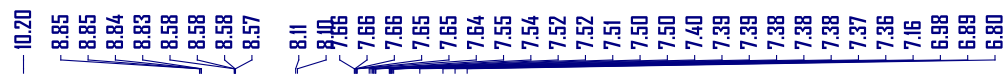

(c)

$3 e$

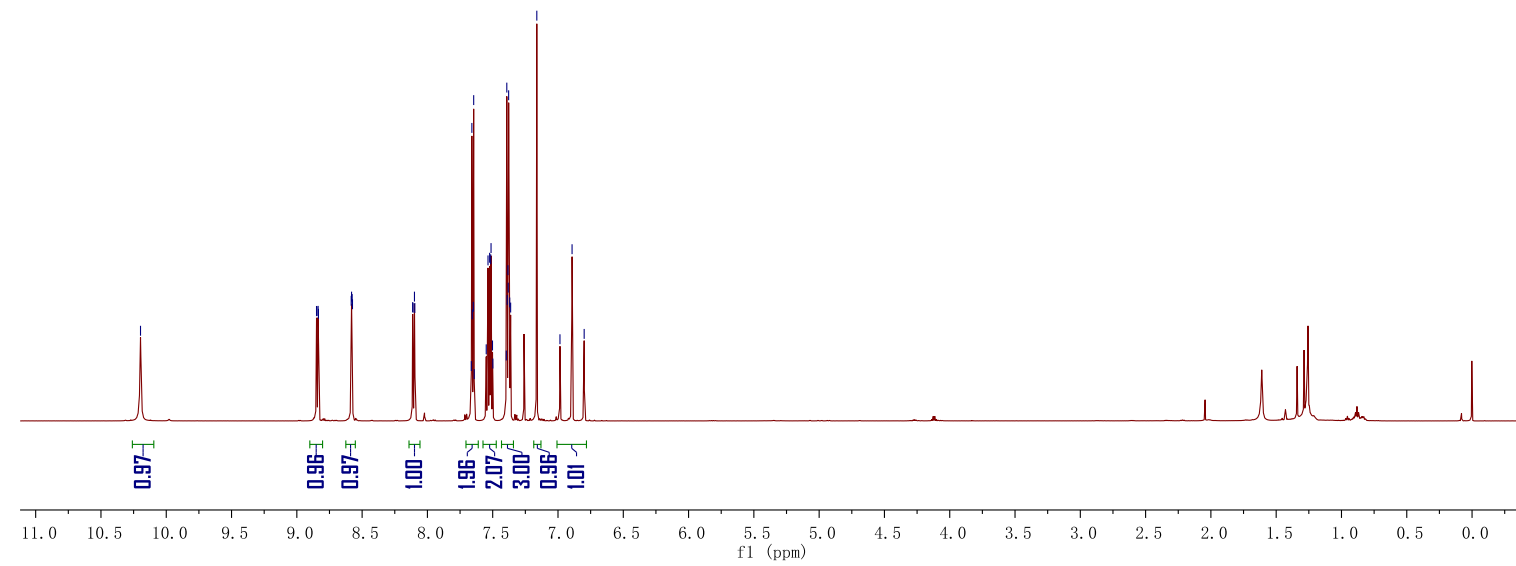

${ }^{13} \mathrm{C}\left\{{ }^{1} \mathrm{H}\right\}$ NMR spectrum of (Z)-2-(4-bromophenyl)-3-((difluoromethyl)thio)-N-(quinolin-8-yl)acrylamide (3e)

$151 \mathrm{MHz}, \mathrm{CDCl}_{3}, 23^{\circ} \mathrm{C}$

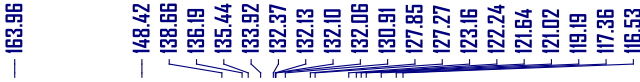<smiles>O=C(Nc1cccc2cccnc12)/C(=C\C(F)F)c1ccc(Br)cc1</smiles>

$3 e$

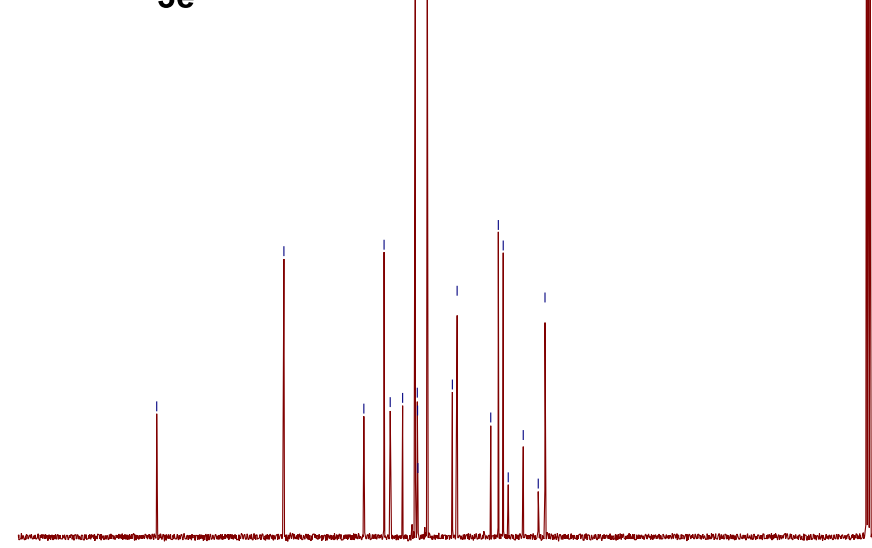

180

170

$160 \quad 150$

140

$120 \quad 110$

190

60

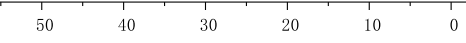


${ }^{19}$ F NMR spectrum of (Z)-2-(4-bromophenyl)-3-((difluoromethyl)thio)-N-(quinolin-8-yl)acrylamide (3e) $565 \mathrm{MHz}, \mathrm{CDCl}_{3}, 23{ }^{\circ} \mathrm{C}$

\section{蛋}<smiles>O=C(Nc1cccc2cccnc12)/C(=C\C(F)F)c1ccc(Br)cc1</smiles>

$3 e$

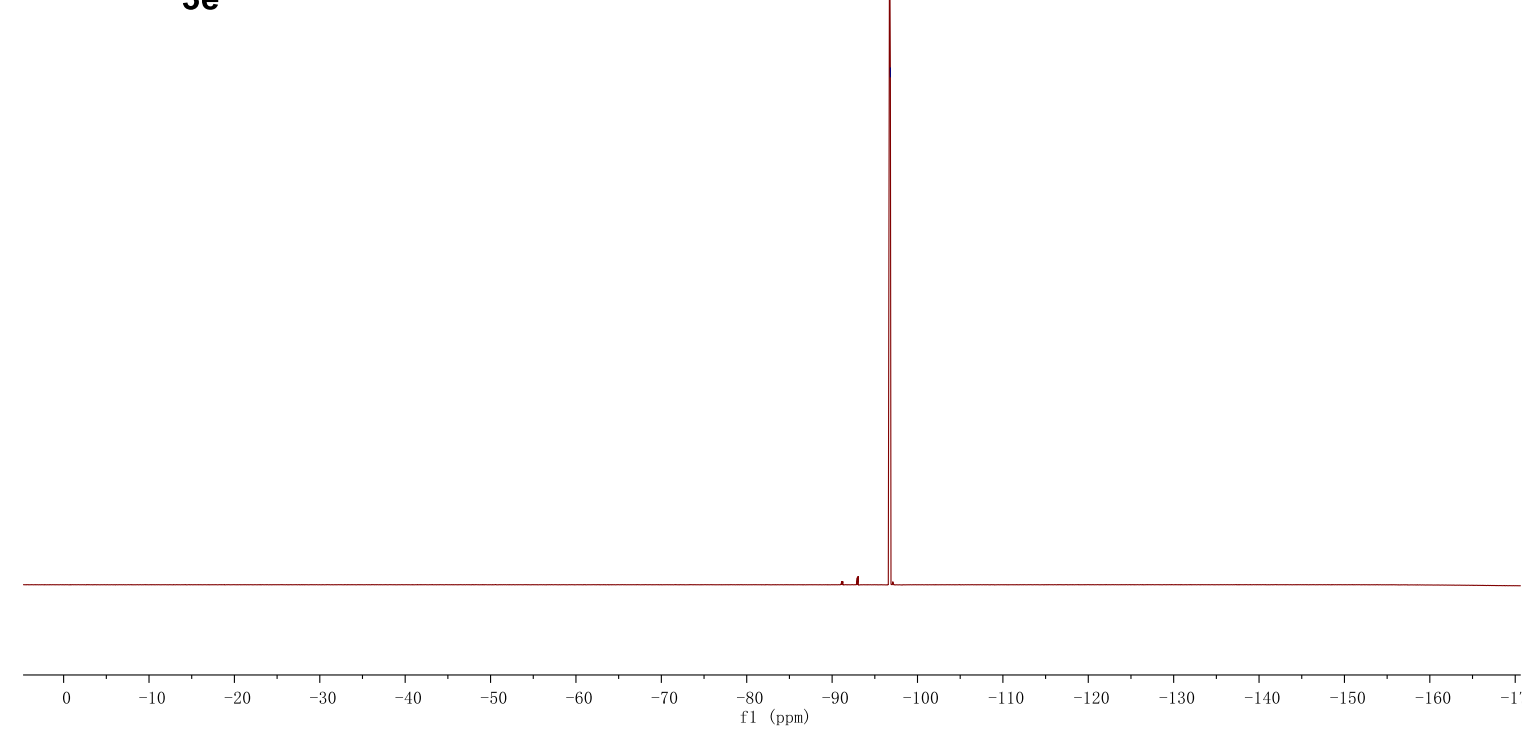

${ }^{1} \mathrm{H}$ NMR spectrum of (Z)-3-((difluoromethyl)thio)-2-(4-iodophenyl)- $N$-(quinolin-8-yl)acrylamide (3f) $600 \mathrm{MHz}, \mathrm{CDCl}_{3}, 23{ }^{\circ} \mathrm{C}$

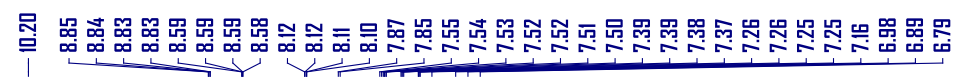<smiles>Cc1cc(/C(=C/SC(F)(F)F)C(=O)Nc2cccc3cccnc23)ccc1I</smiles>

$3 f$

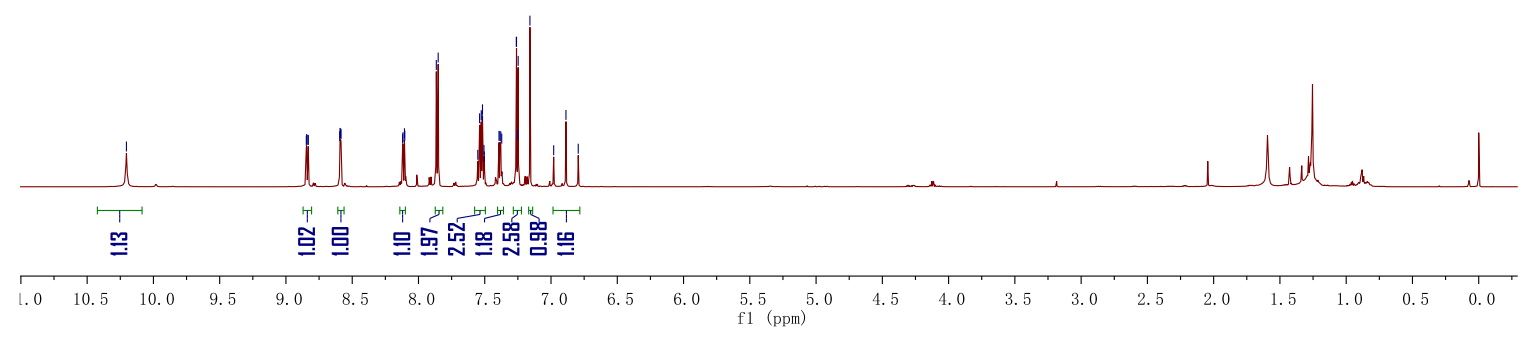


${ }^{13} \mathrm{C}\left\{{ }^{1} \mathrm{H}\right\}$ NMR spectrum of (Z)-3-((difluoromethyl)thio)-2-(4-iodophenyl)-N-(quinolin-8-yl)acrylamide (3f) $151 \mathrm{MHz}, \mathrm{CDCl}_{3}, 23{ }^{\circ} \mathrm{C}$
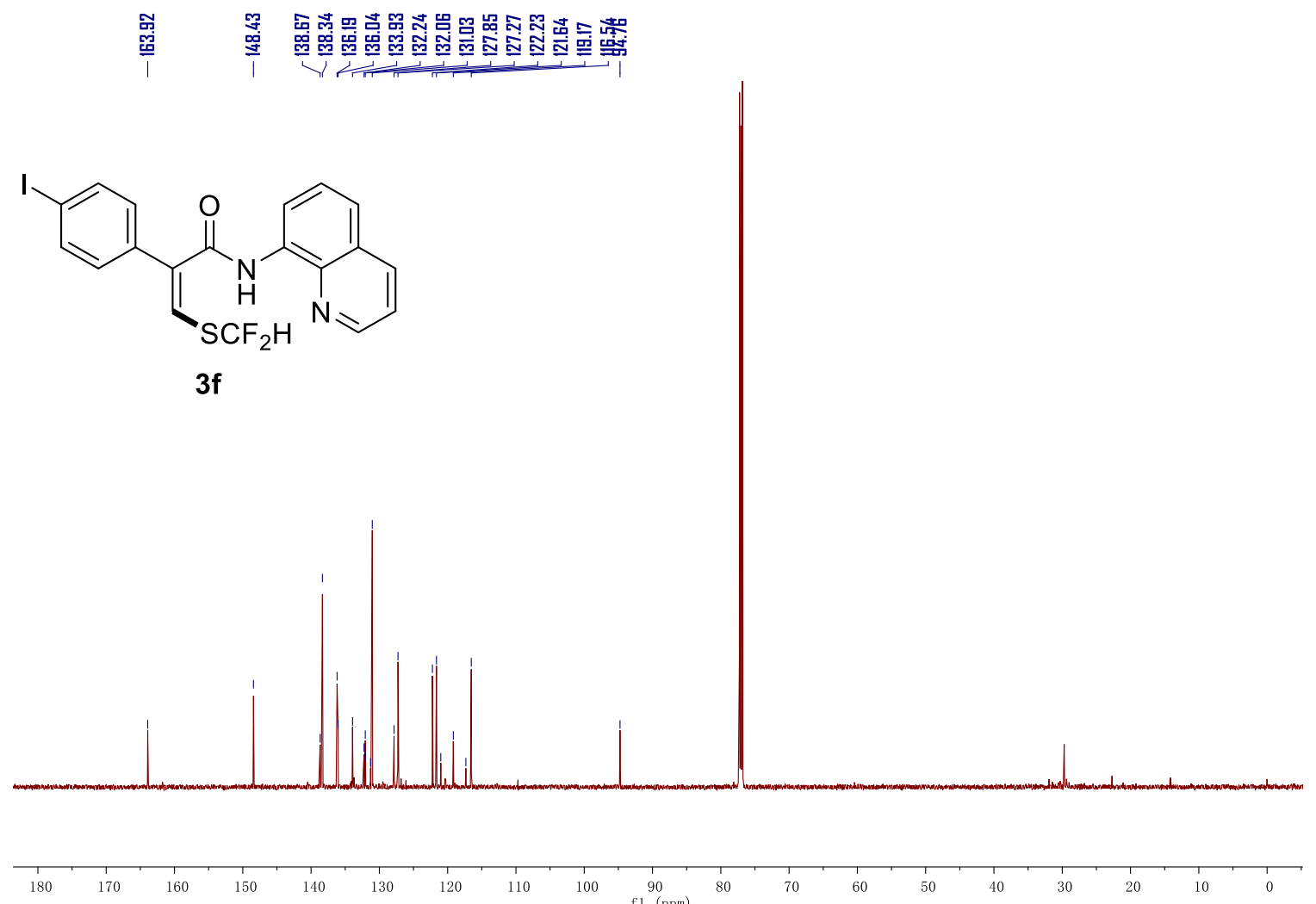

${ }^{19} \mathrm{~F}$ NMR spectrum of (Z)-3-((difluoromethyl)thio)-2-(4-iodophenyl)-N-(quinolin-8-yl)acrylamide (3f) $565 \mathrm{MHz}, \mathrm{CDCl}_{3}, 23{ }^{\circ} \mathrm{C}$<smiles>O=C(Nc1cccc2cccnc12)/C(=C\C(F)F)c1ccc(I)cc1</smiles>

$3 f$
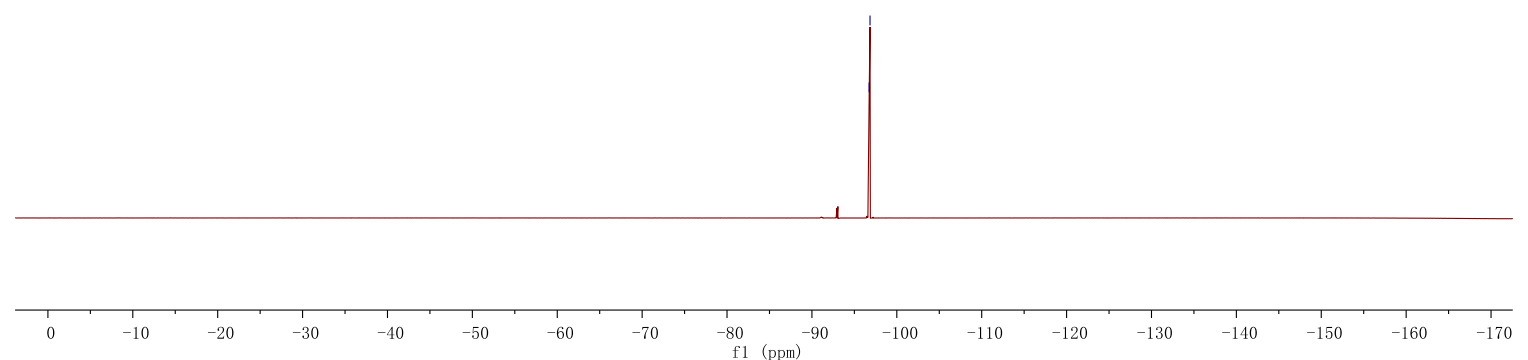
${ }^{1} \mathrm{H}$ NMR spectrum of (Z)-2-(4-cyanophenyl)-3-((difluoromethyl)thio)-N-(quinolin-8-yl)acrylamide (3g)

$600 \mathrm{MHz}, \mathrm{CDCl}_{3}, 23{ }^{\circ} \mathrm{C}$

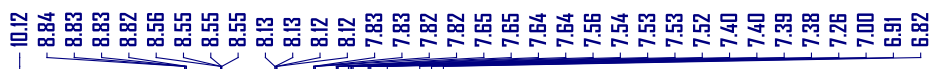

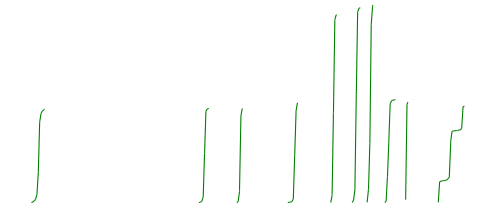

(C)

$3 g$

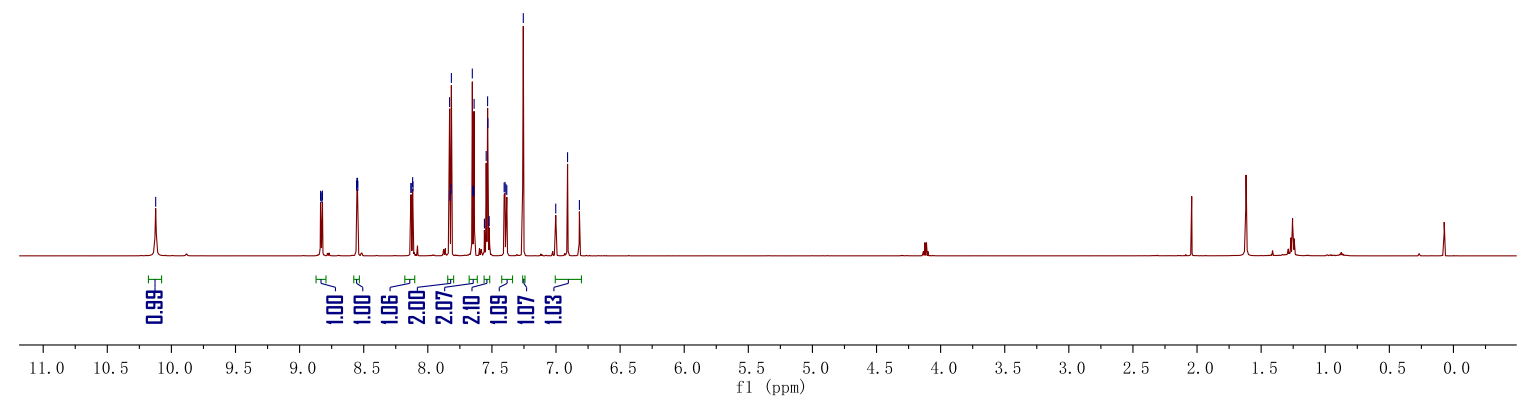

${ }^{13} \mathrm{C}\left\{{ }^{1} \mathrm{H}\right\}$ NMR spectrum of (Z)-2-(4-cyanophenyl)-3-((difluoromethyl)thio)-N-(quinolin-8-yl)acrylamide (3g) $151 \mathrm{MHz}, \mathrm{CDCl}_{3}, 23{ }^{\circ} \mathrm{C}$

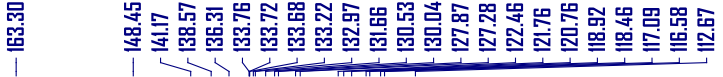<smiles>N#Cc1ccc(/C(=C/SC(F)(F)F)C(=O)Nc2cccc3cccnc23)cc1</smiles>

$3 g$

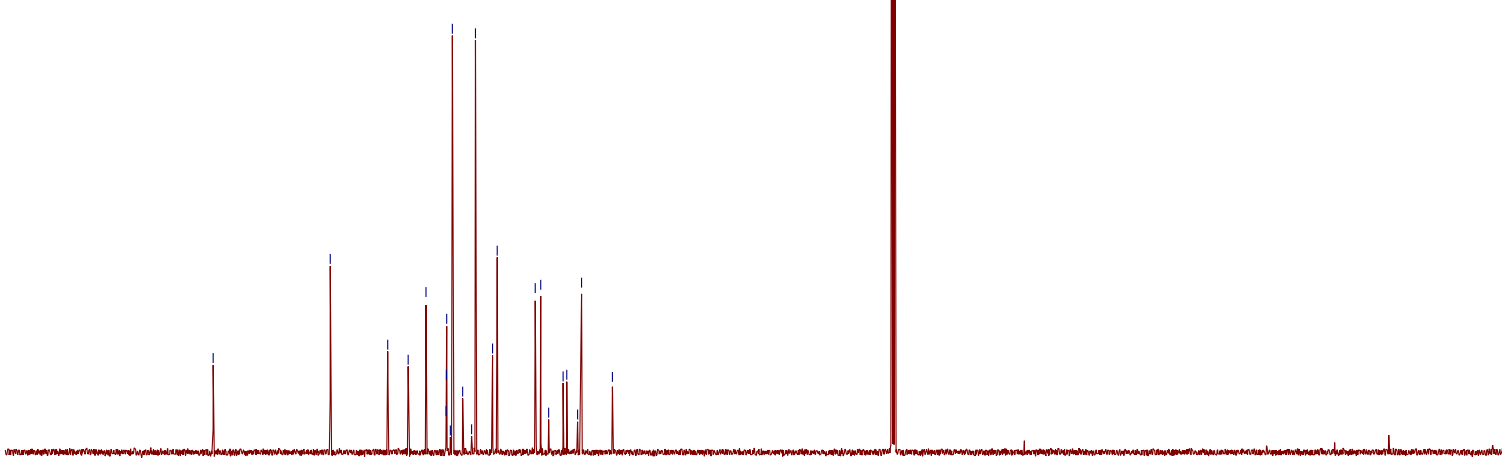

$\begin{array}{lllllllll}180 & 170 & 160 & 150 & 140 & 130 & 120 & 110 & 100 \\ \mathrm{f} 1(\mathrm{ppm}) & 90\end{array}$ 
${ }^{19} \mathrm{~F}$ NMR spectrum of (Z)-2-(4-cyanophenyl)-3-((difluoromethyl)thio)- $\mathrm{N}$-(quinolin-8-yl)acrylamide (3g) $565 \mathrm{MHz}, \mathrm{CDCl}_{3}, 23{ }^{\circ} \mathrm{C}$

\section{空}<smiles>N#Cc1ccc(C(=C[SeH])C(=O)Nc2cccc3cccnc23)cc1</smiles>

$3 g$

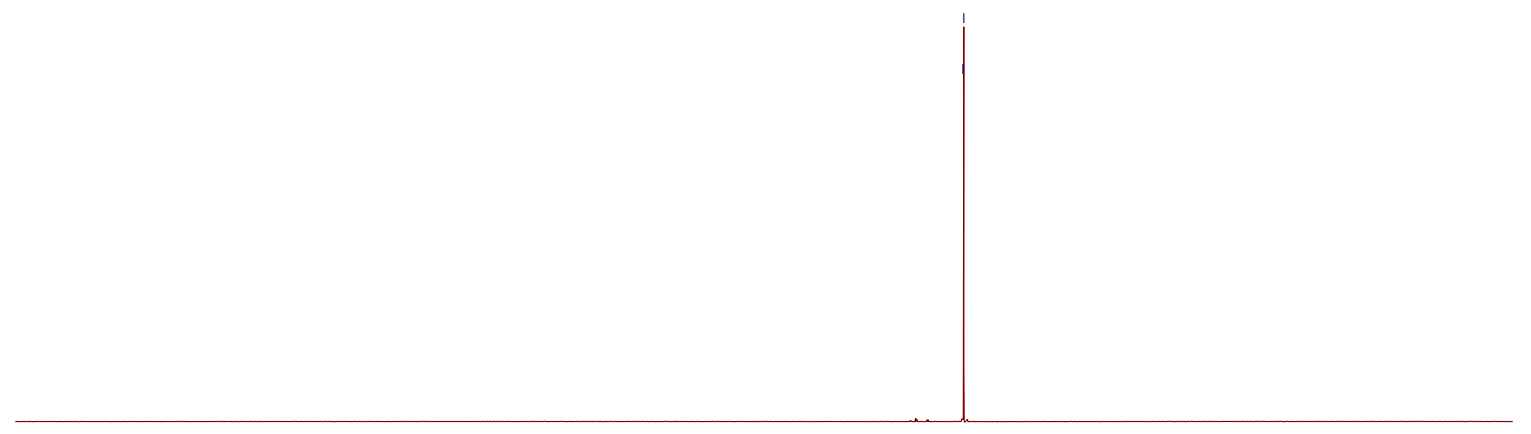

\begin{tabular}{llllllllllllllllllllllllllllllllllll}
\hline 0 & -5 & -10 & -15 & -20 & -25 & -30 & -35 & -40 & -45 & -50 & -55 & -60 & -65 & -70 & -75 & -80 & -85 & -90 & -95 & -105 & -115 & -125 & -135 & -145 & \\
& & & &
\end{tabular}

${ }^{1} \mathrm{H}$ NMR spectrum of (Z)-3-((difluoromethyl)thio)-N-(quinolin-8-yl)-2-(4-(trifluoromethyl)phenyl)acrylamide (3h) $600 \mathrm{MHz}, \mathrm{CDCl}_{3}, 23{ }^{\circ} \mathrm{C}$

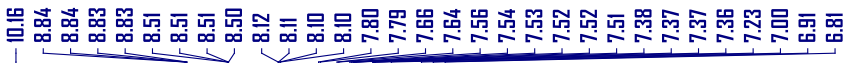

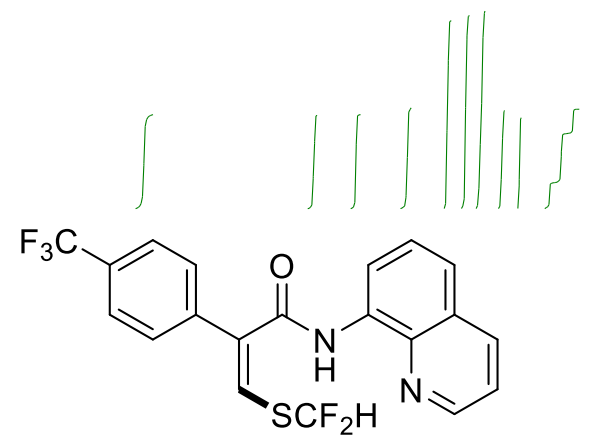

$3 \mathrm{~h}$

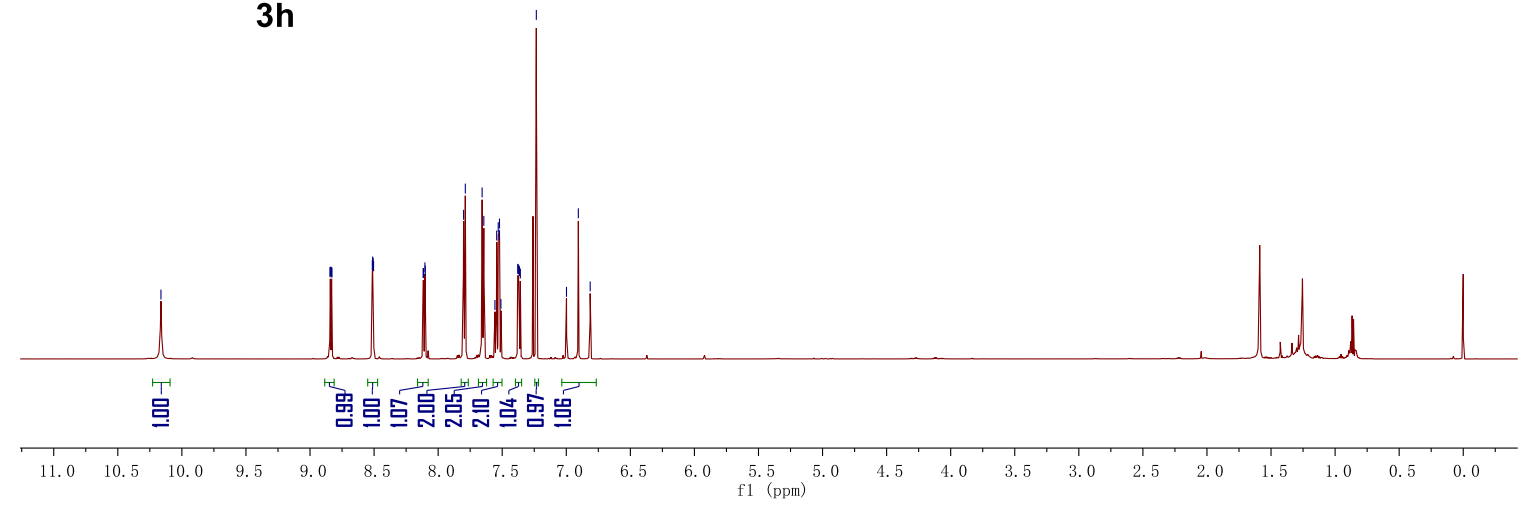


${ }^{13} \mathrm{C}\left\{{ }^{1} \mathrm{H}\right\}$ NMR spectrum of (Z)-3-((difluoromethyl)thio)-N-(quinolin-8-yl)-2-(4-(trifluoromethyl)phenyl)acrylamide (3h)

$151 \mathrm{MHz}, \mathrm{CDCl}_{3}, 23^{\circ} \mathrm{C}$

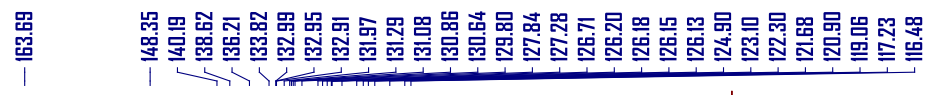<smiles>O=C(Nc1cccc2cccnc12)/C(=C\C(F)F)c1ccc(C(F)(F)F)cc1</smiles>

$3 \mathbf{h}$

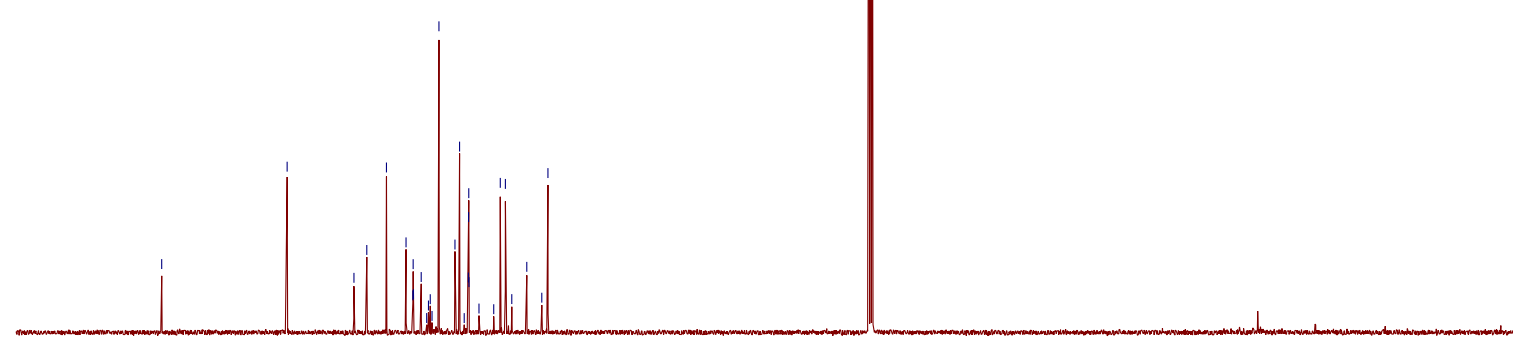

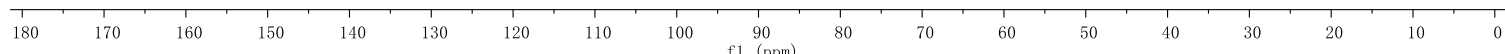

${ }^{19} \mathrm{~F}$ NMR spectrum of (Z)-3-((difluoromethyl)thio)-N-(quinolin-8-yl)-2-(4-(trifluoromethyl)phenyl)acrylamide (3h) $565 \mathrm{MHz}, \mathrm{CDCl}_{3}, 23{ }^{\circ} \mathrm{C}$

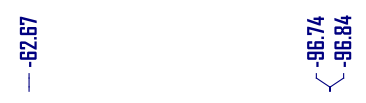

(c)

$3 h$

\begin{tabular}{|c|c|c|c|c|c|c|c|c|c|c|c|c|c|c|c|c|}
\hline 0 & -10 & -20 & -30 & -40 & -50 & -60 & -70 & $\begin{array}{l}-80 \\
\mathrm{fl}^{-80}(\mathrm{pm}) \\
17\end{array}$ & -90 & -100 & -110 & -120 & -130 & -140 & -150 & -160 \\
\hline
\end{tabular}


${ }^{1} \mathrm{H}$ NMR spectrum of (Z)-2-([1,1'-biphenyl]-4-yl)-3-((difluoromethyl)thio)-N-(quinolin-8-yl)acrylamide (3i) $600 \mathrm{MHz}, \mathrm{CDCl}_{3}, 23{ }^{\circ} \mathrm{C}$

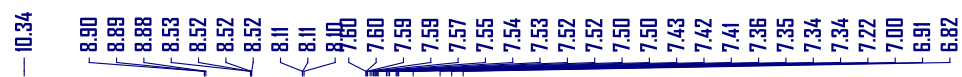<smiles>C#CC1CCCCC1CC#CC</smiles>

$3 \mathbf{i}$

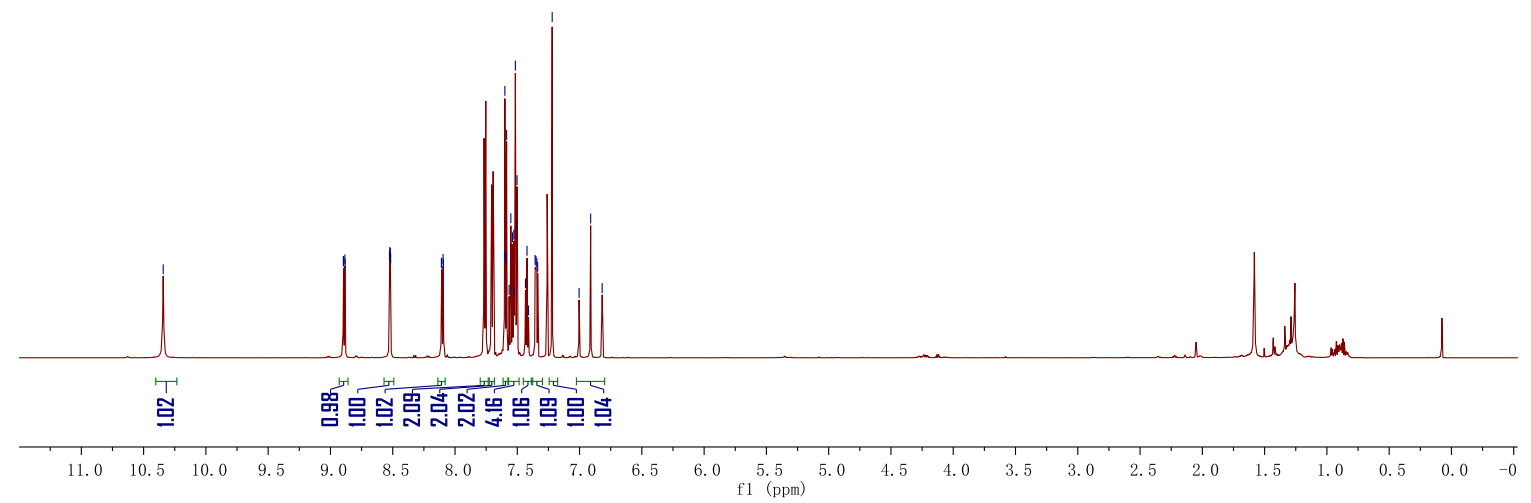

${ }^{13} \mathrm{C}\left\{{ }^{1} \mathrm{H}\right\}$ NMR spectrum of (Z)-2-([1,1'-biphenyl]-4-yl)-3-((difluoromethyl)thio)-N-(quinolin-8-yl)acrylamide (3i) $151 \mathrm{MHz}, \mathrm{CDCl}_{3}, 23{ }^{\circ} \mathrm{C}$

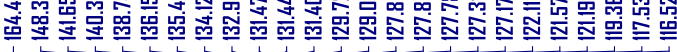<smiles>O=C(Nc1cccc2cccnc12)/C(=C\C(F)F)c1ccc(-c2ccccc2)cc1</smiles>

3i

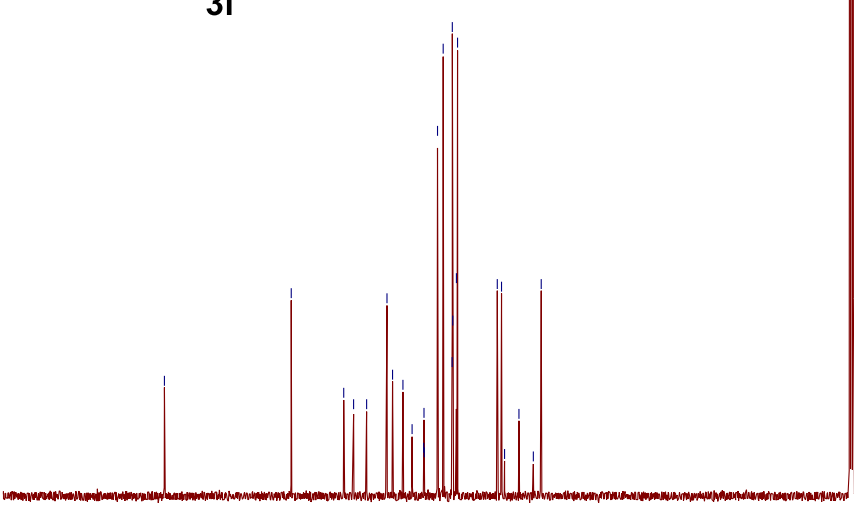

$180 \quad 170$

150

120

$10 \quad 100$

$1 \stackrel{90}{(\mathrm{ppm})}$ 
${ }^{19} \mathrm{~F}$ NMR spectrum of (Z)-2-([1,1'-biphenyl]-4-yl)-3-((difluoromethyl)thio)-N-(quinolin-8-yl)acrylamide (3i) $565 \mathrm{MHz}, \mathrm{CDCl}_{3}, 23{ }^{\circ} \mathrm{C}$<smiles>O=C(Nc1cccc2cccnc12)/C(=C\C(F)F)c1ccc(-c2ccccc2)cc1</smiles>

$3 \mathbf{i}$

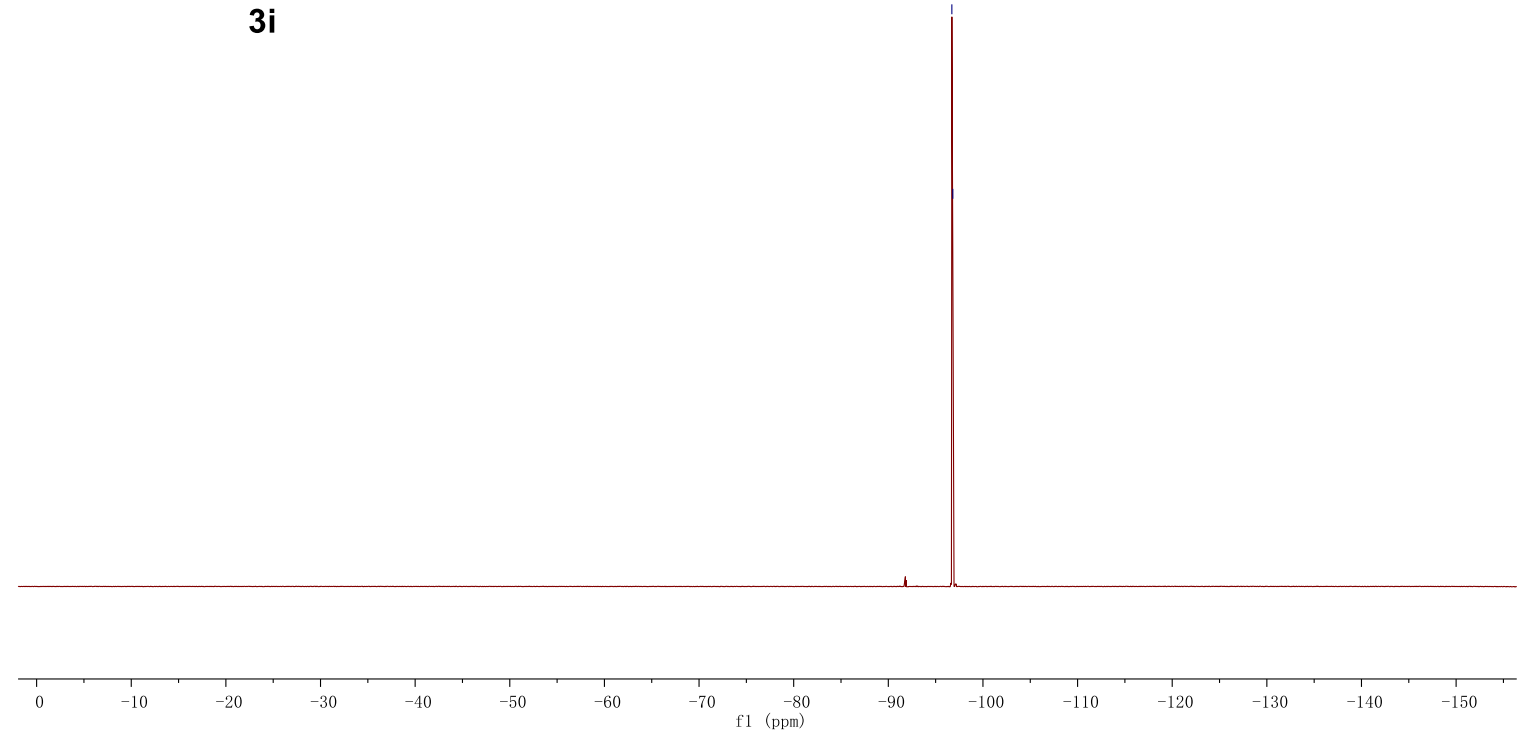

${ }^{1} \mathrm{H}$ NMR spectrum of (Z)-3-((difluoromethyl)thio)-2-(4-methoxyphenyl)-N-(quinolin-8-yl)acrylamide (3j) $600 \mathrm{MHz}, \mathrm{CDCl}_{3}, 23{ }^{\circ} \mathrm{C}$

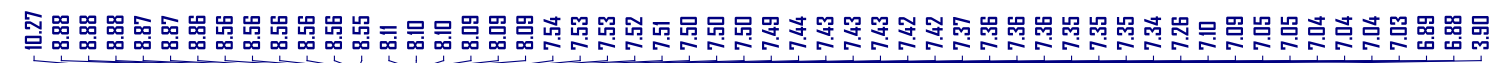

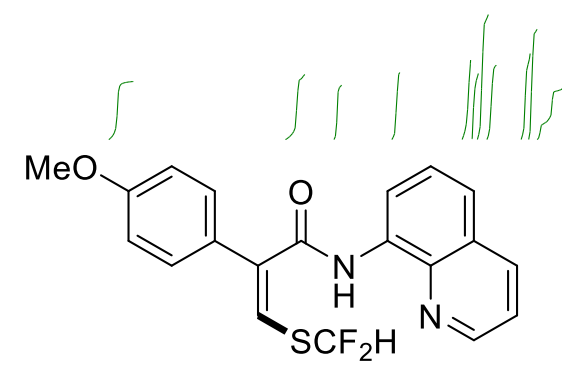

3j

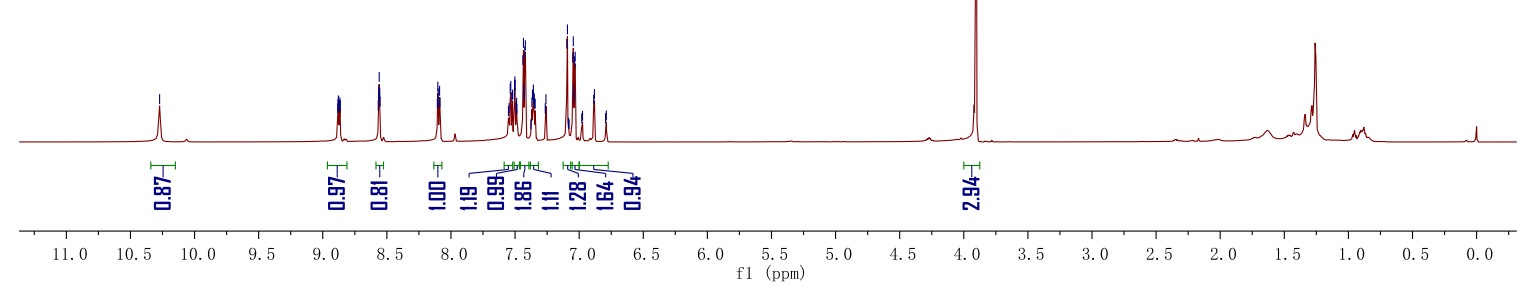


${ }^{13} \mathrm{C}\left\{{ }^{1} \mathrm{H}\right\}$ NMR spectrum of (Z)-3-((difluoromethyl)thio)-2-(4-methoxyphenyl)-N-(quinolin-8-yl)acrylamide (3j) $151 \mathrm{MHz}, \mathrm{CDCl}_{3}, 23{ }^{\circ} \mathrm{C}$

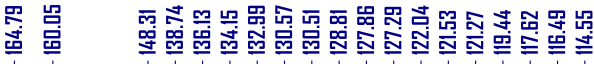
密

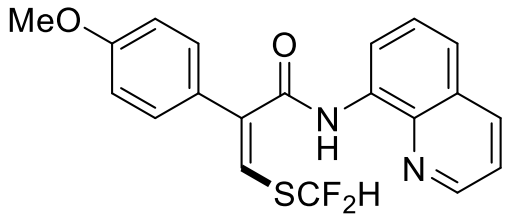

$3 \mathbf{j}$
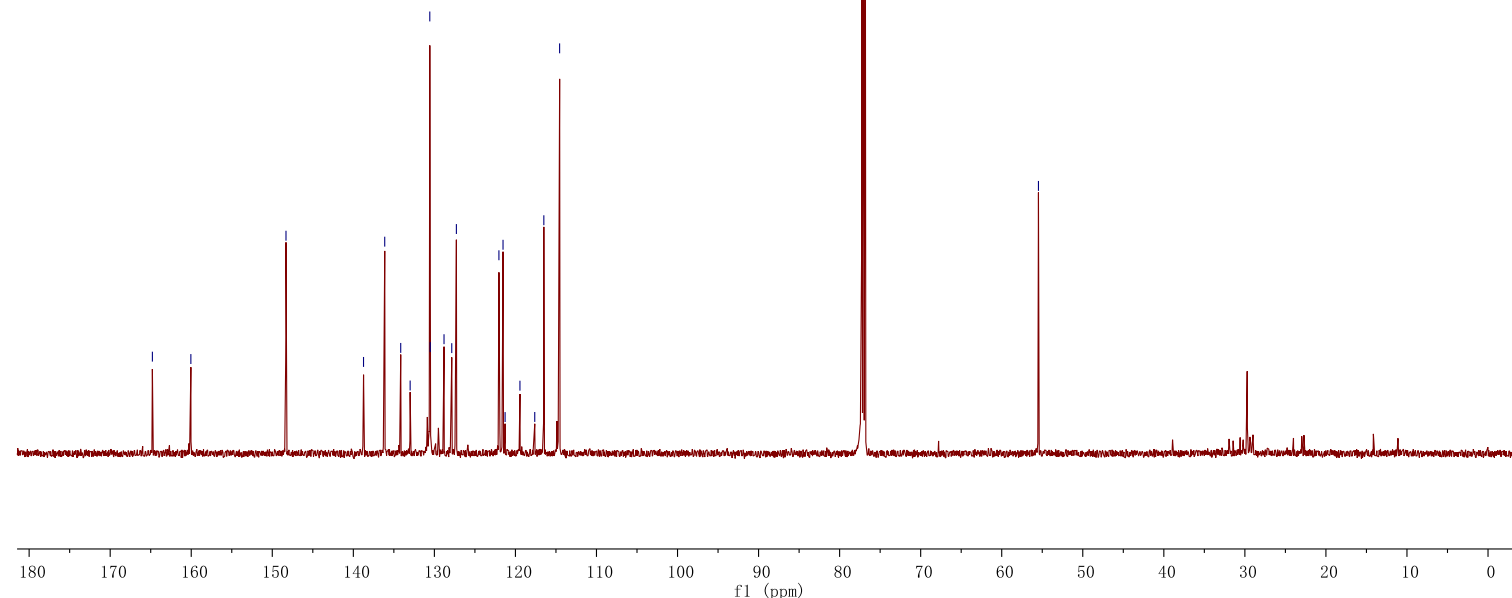

${ }^{19} \mathrm{~F}$ NMR spectrum of (Z)-3-((difluoromethyl)thio)-2-(4-methoxyphenyl)-N-(quinolin-8-yl)acrylamide (3j) $565 \mathrm{MHz}, \mathrm{CDCl}_{3}, 23{ }^{\circ} \mathrm{C}$

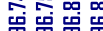

证

(N)

3j

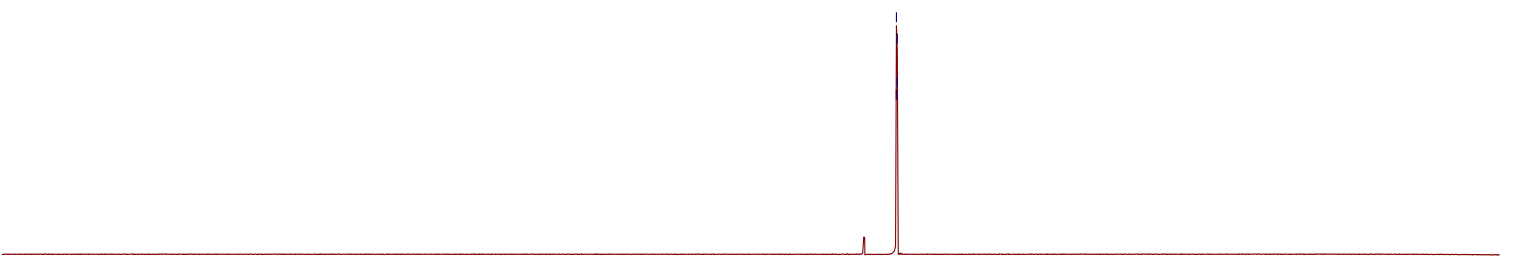

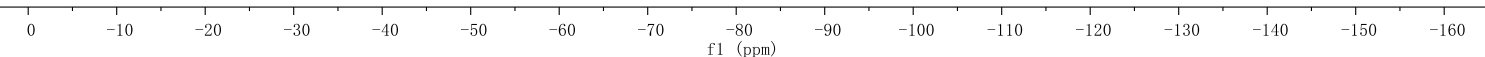


${ }^{1} \mathrm{H}$ NMR spectrum of (Z)-3-((difluoromethyl)thio)-2-(3-methoxyphenyl)-N-(quinolin-8-yl)acrylamide (3k) $600 \mathrm{MHz}, \mathrm{CDCl}_{3}, 23{ }^{\circ} \mathrm{C}$

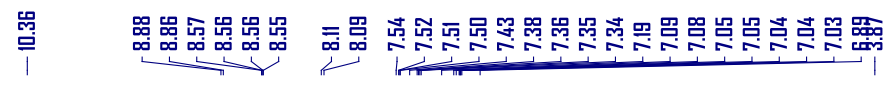<smiles>COC(=O)C=C(C(=O)Nc1cccc2cccnc12)c1cccc(OC)c1</smiles>

$3 \mathbf{k}$

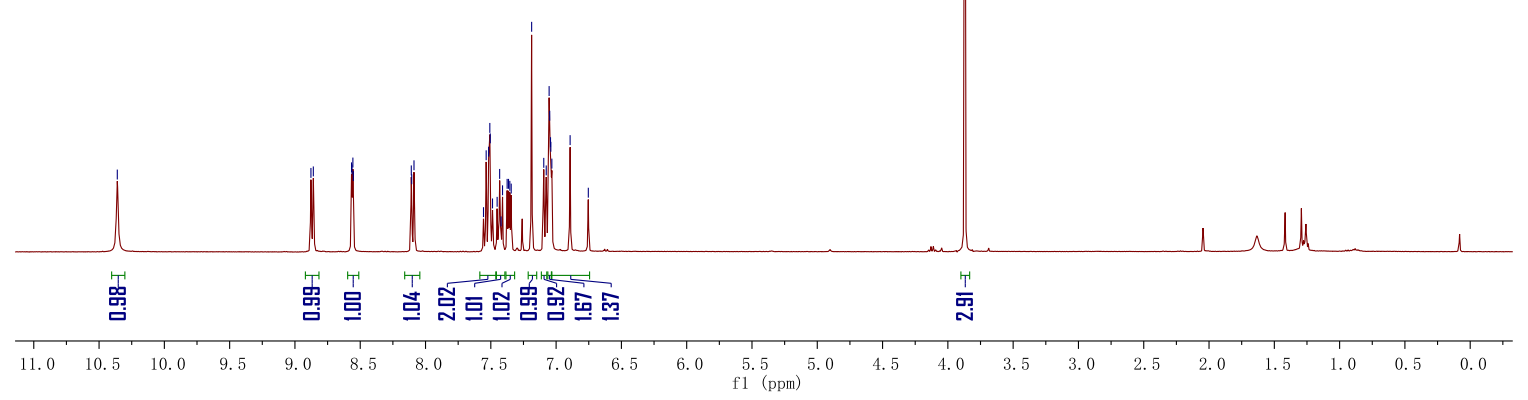

${ }^{13} \mathrm{C}\left\{{ }^{1} \mathrm{H}\right\}$ NMR spectrum of (Z)-3-((difluoromethyl)thio)-2-(3-methoxyphenyl)-N-(quinolin-8-yl)acrylamide (3k) $151 \mathrm{MHz}, \mathrm{CDCl}_{3}, 23{ }^{\circ} \mathrm{C}$

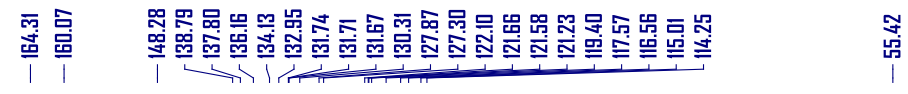<smiles>COc1cccc(C(=CSC(F)(F)F)C(=O)Nc2cccc3cccnc23)c1</smiles>

3k

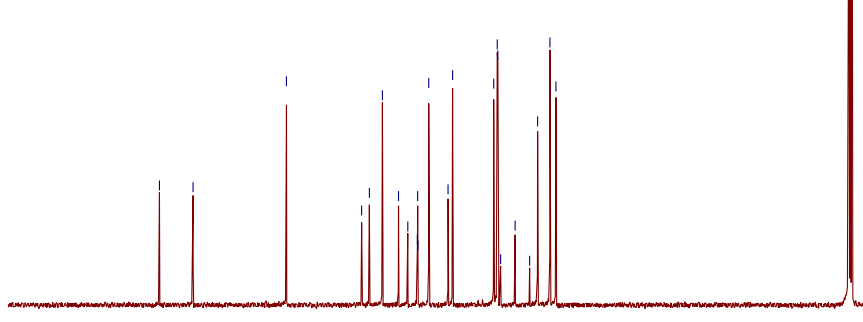


${ }^{19} \mathrm{~F}$ NMR spectrum of (Z)-3-((difluoromethyl)thio)-2-(3-methoxyphenyl)-N-(quinolin-8-yl)acrylamide (3k) $565 \mathrm{MHz}, \mathrm{CDCl}_{3}, 23{ }^{\circ} \mathrm{C}$<smiles>COc1cccc(/C(=C/C(F)F)C(=O)Nc2cccc3cccnc23)c1</smiles>

$3 \mathbf{k}$
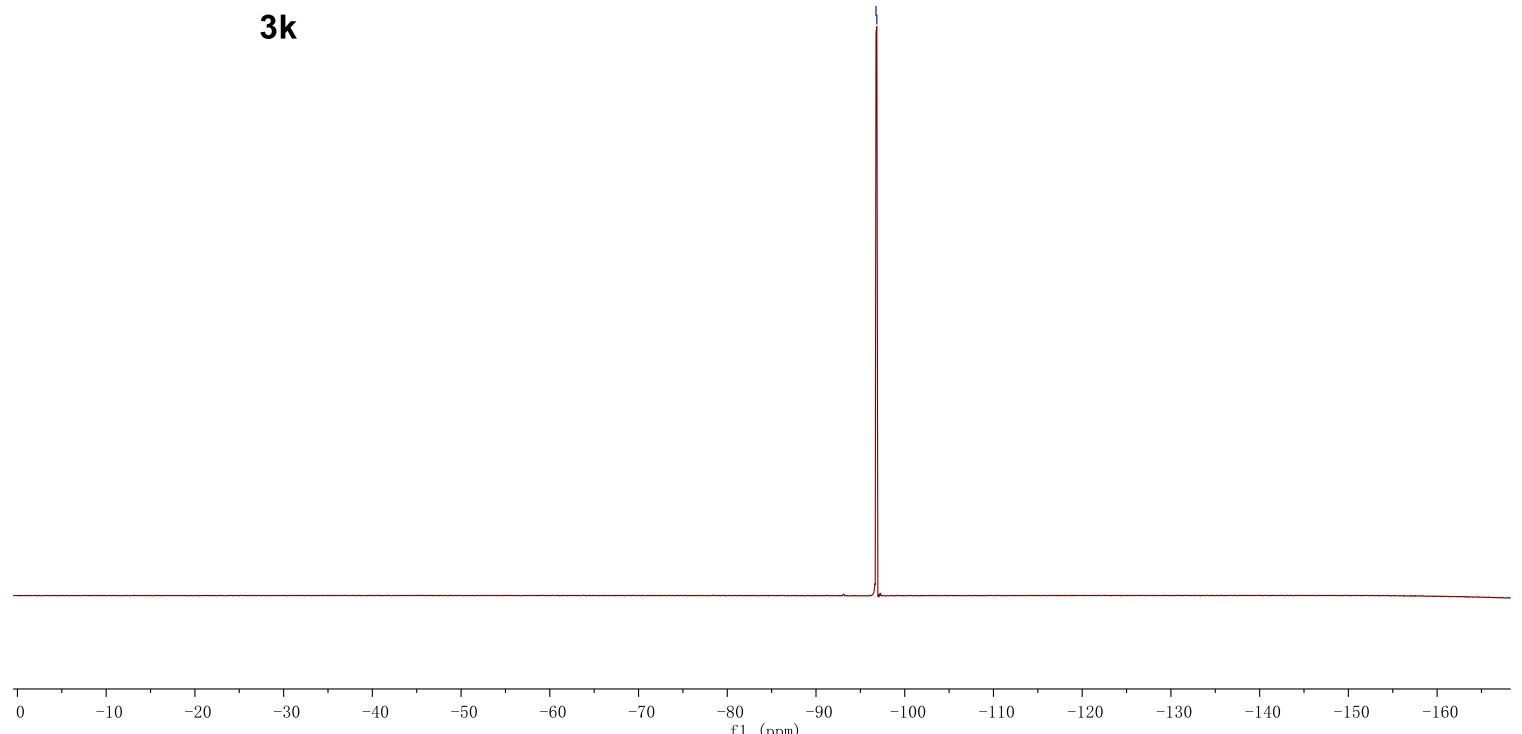

${ }^{1} \mathrm{H}$ NMR spectrum of (Z)-3-((difluoromethyl)thio)-N-(quinolin-8-yl)-2-(o-tolyl)acrylamide (3I) $600 \mathrm{MHz}, \mathrm{CDCl}_{3}, 23{ }^{\circ} \mathrm{C}$

总<smiles>CCc1ccccc1/C(=C/[SH+](F)(F)F)C(=O)Nc1cccc2cccnc12</smiles>

31

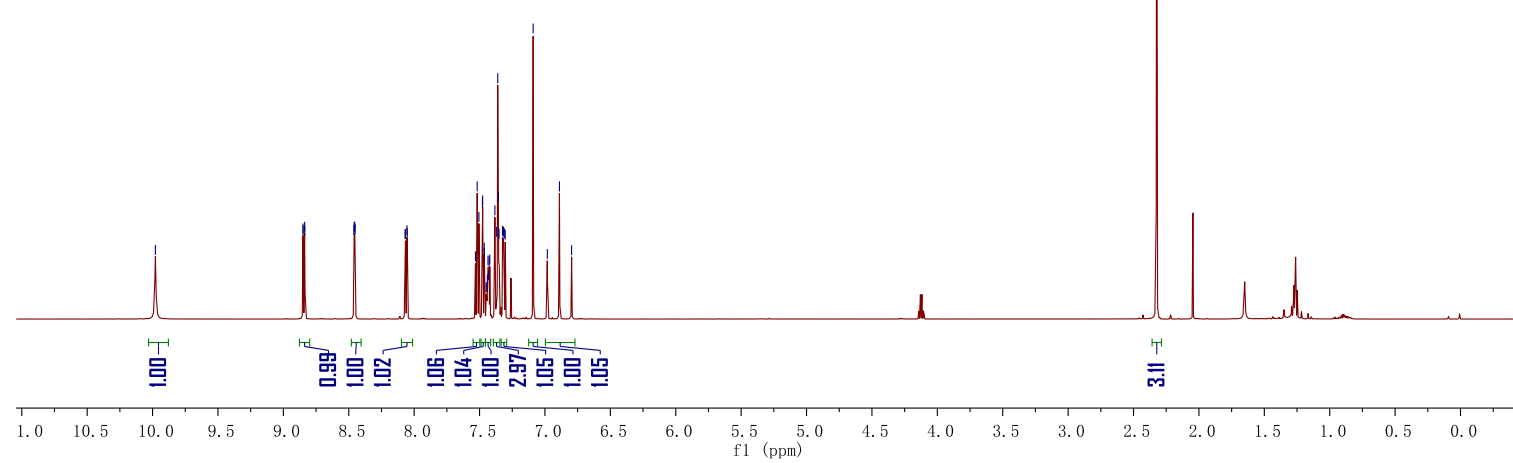


${ }^{13} \mathrm{C}\left\{{ }^{1} \mathrm{H}\right\}$ NMR spectrum of (Z)-3-((difluoromethyl)thio)-N-(quinolin-8-yl)-2-(o-tolyl)acrylamide (3I) $151 \mathrm{MHz}, \mathrm{CDCl}_{3}, 23{ }^{\circ} \mathrm{C}$

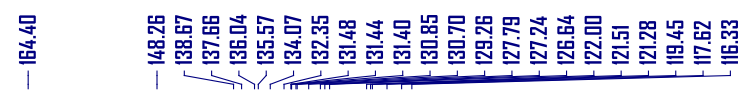

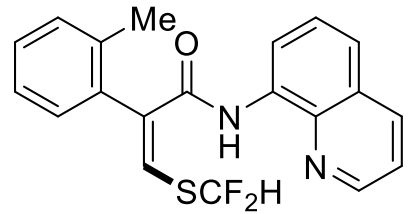

3I
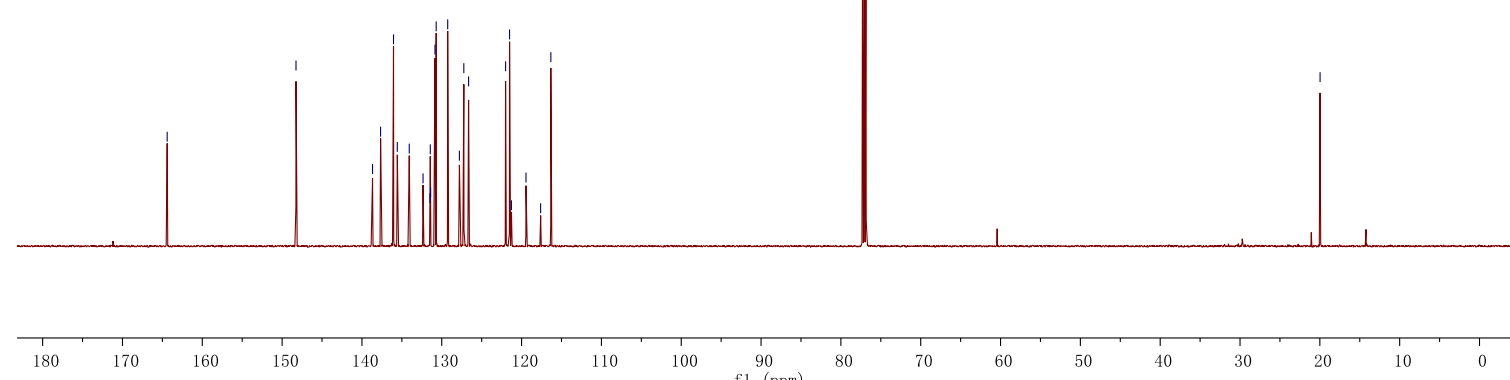

${ }^{19} \mathrm{~F}$ NMR spectrum of (Z)-3-((difluoromethyl)thio)-N-(quinolin-8-yl)-2-(o-tolyl)acrylamide (3I) $565 \mathrm{MHz}, \mathrm{CDCl}_{3}, 23^{\circ} \mathrm{C}$<smiles>Cc1ccccc1/C(=C/SC(F)(F)F)C(=O)Nc1cccc2cccnc12</smiles>

3 I

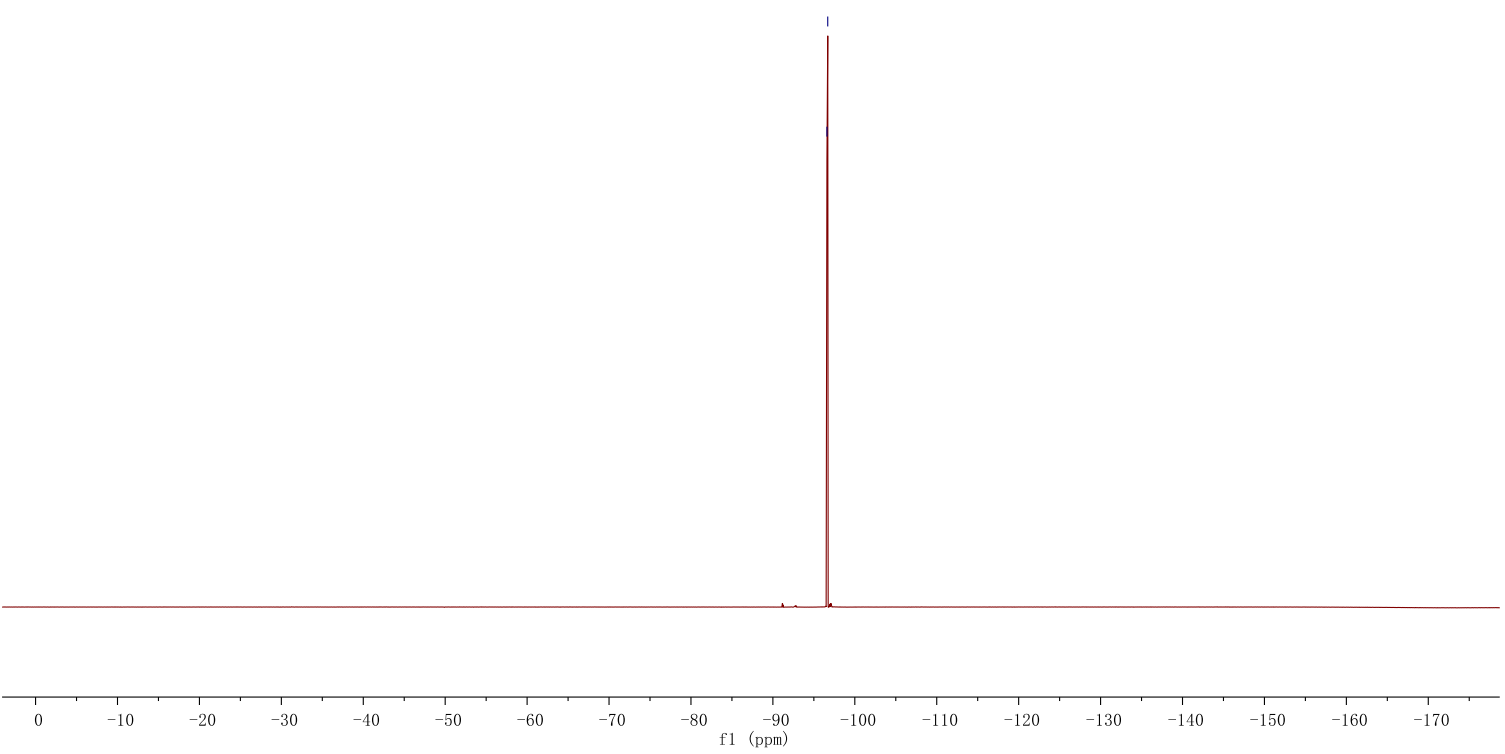


${ }^{1} \mathrm{H}$ NMR spectrum of (Z)-3-((difluoromethyl)thio)-2-(2-iodophenyl)-N-(quinolin-8-yl)acrylamide (3m) $600 \mathrm{MHz}, \mathrm{CDCl}_{3}, 23{ }^{\circ} \mathrm{C}$

螘<smiles>O=C(Nc1cccc2cccnc12)/C(=C\C(F)F)c1ccccc1I</smiles>

$3 \mathrm{~m}$

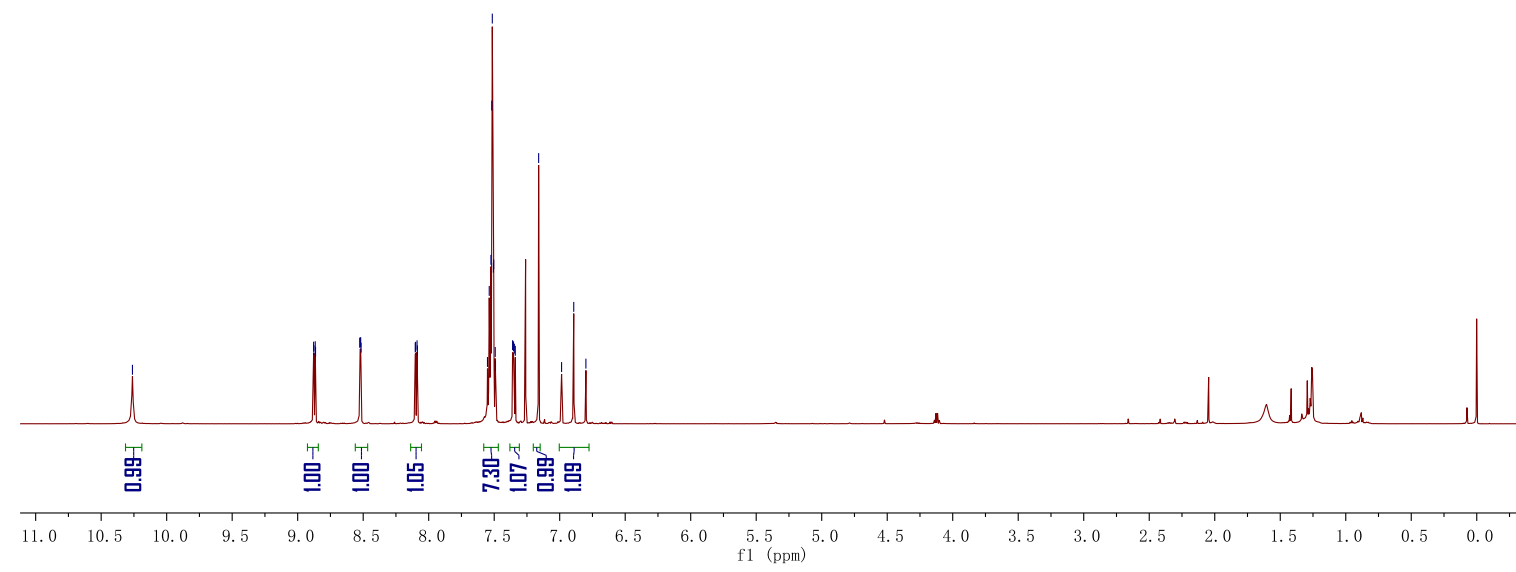

${ }^{13} \mathrm{C}\left\{{ }^{1} \mathrm{H}\right\}$ NMR spectrum of (Z)-3-((difluoromethyl)thio)-2-(2-iodophenyl)-N-(quinolin-8-yl)acrylamide (3m) $151 \mathrm{MHz}, \mathrm{CDCl}_{3}, 23{ }^{\circ} \mathrm{C}$

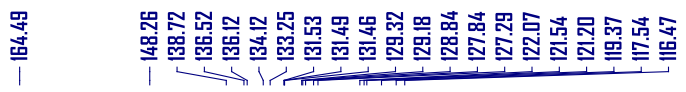<smiles>O=C(Nc1cccc2cccnc12)/C(=C\C(F)F)c1ccccc1I</smiles>

$3 m$

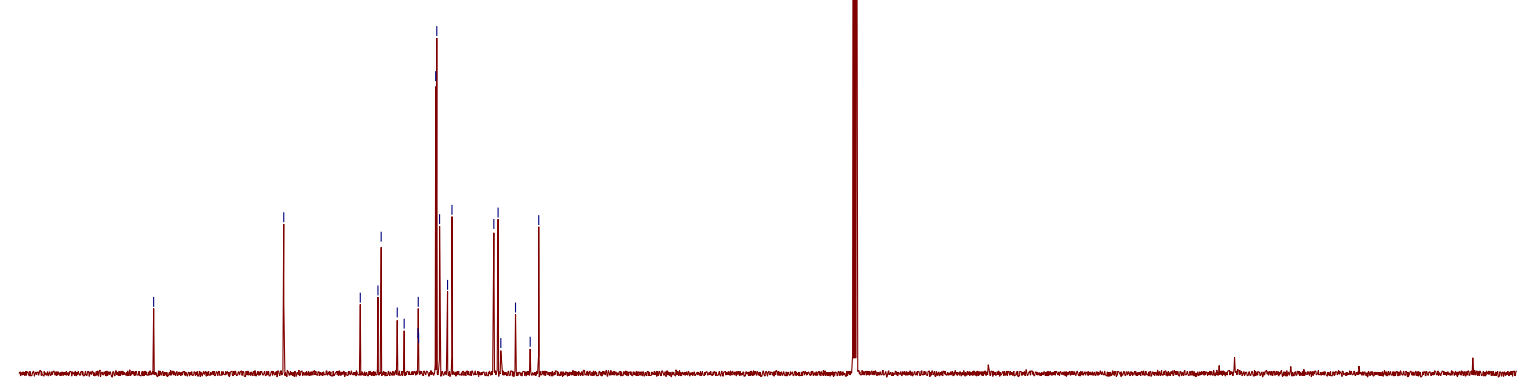

180

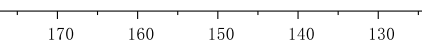

120

$110 \quad 100$ $\mathrm{fl}^{90}(\mathrm{ppm}) \quad 80$ 
${ }^{19} \mathrm{~F}$ NMR spectrum of (Z)-3-((difluoromethyl)thio)-2-(2-iodophenyl)-N-(quinolin-8-yl)acrylamide (3m) $565 \mathrm{MHz}, \mathrm{CDCl}_{3}, 23{ }^{\circ} \mathrm{C}$<smiles>O=C(Nc1cccc2cccnc12)/C(=C\C(F)F)c1ccccc1I</smiles>

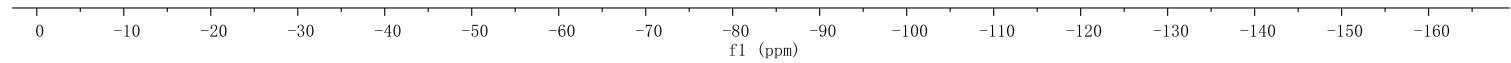

${ }^{1} \mathrm{H}$ NMR spectrum of (Z)-2-(benzo[d][1,3]dioxol-5-yl)-3-((difluoromethyl)thio)-N-(quinolin-8-yl)acrylamide (3n) $600 \mathrm{MHz}, \mathrm{CDCl}_{3}, 23{ }^{\circ} \mathrm{C}$

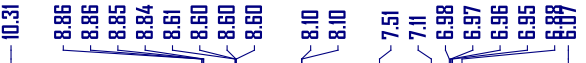

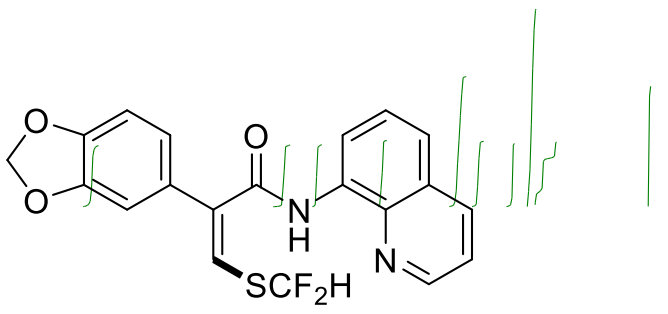

$3 n$

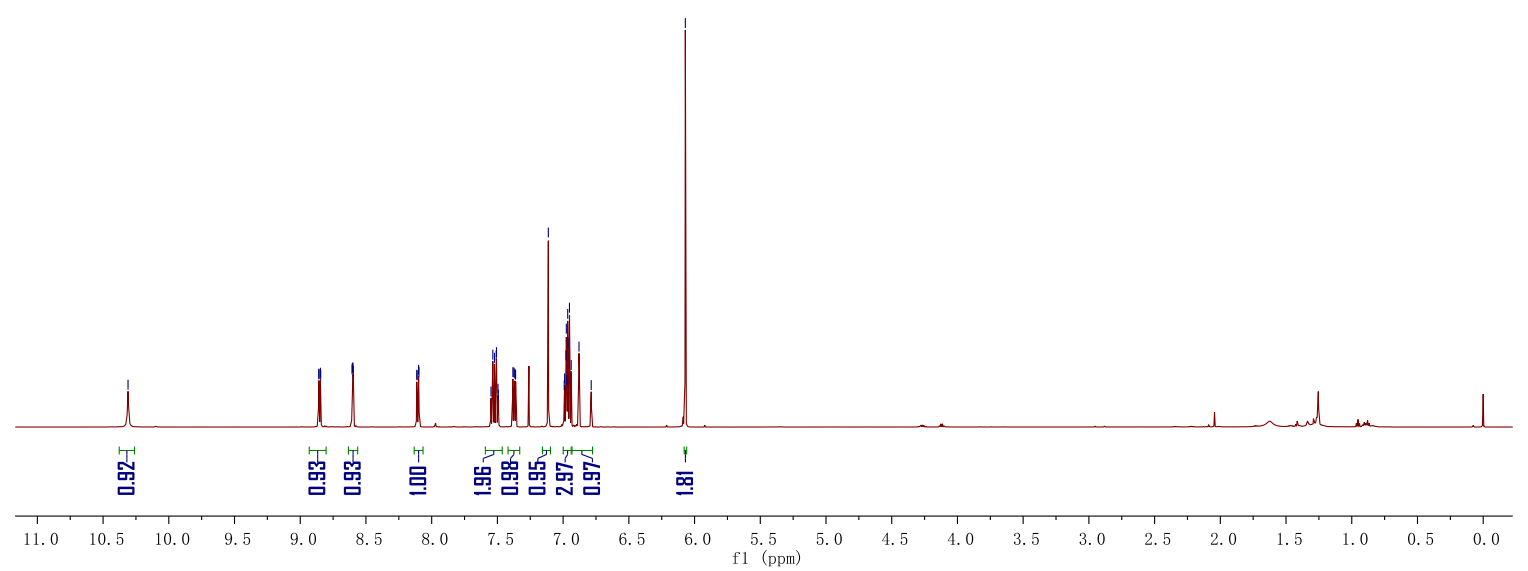


${ }^{13} \mathrm{C}\left\{{ }^{1} \mathrm{H}\right\}$ NMR spectrum of (Z)-2-(benzo[d][1,3]dioxol-5-yl)-3-((difluoromethyl)thio)-N-(quinolin-8-yl)acrylamide (3n)

$151 \mathrm{MHz}, \mathrm{CDCl}_{3}, 23{ }^{\circ} \mathrm{C}$

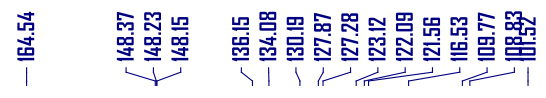<smiles>O=C(Nc1cccc2cccnc12)/C(=C\C(F)F)c1ccc2c(c1)OCO2</smiles>

$3 n$

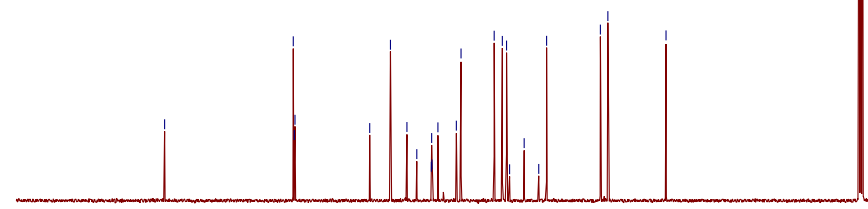

$\begin{array}{lllllllllllllllll}1 & 180 & 170 & 160 & 150 & 140 & 130 & 120 & 110 & 100 & 90 & 1 & 1 & 1 & 1 & 1 & 1 \\ \mathrm{f} 1(\mathrm{pmm}) & 80 & 70 & 60 & 50 & 40 & 30 & 20 & 10 & 0\end{array}$

${ }^{19}$ F NMR spectrum of (Z)-2-(benzo[d][1,3]dioxol-5-yl)-3-((difluoromethyl)thio)-N-(quinolin-8-yl)acrylamide (3n) $565 \mathrm{MHz}, \mathrm{CDCl}_{3}, 23{ }^{\circ} \mathrm{C}$<smiles>CC(F)[SiH2]/C=C(\C(=O)Nc1cccc2cccnc12)c1ccc2c(c1)OCO2</smiles>

$3 n$

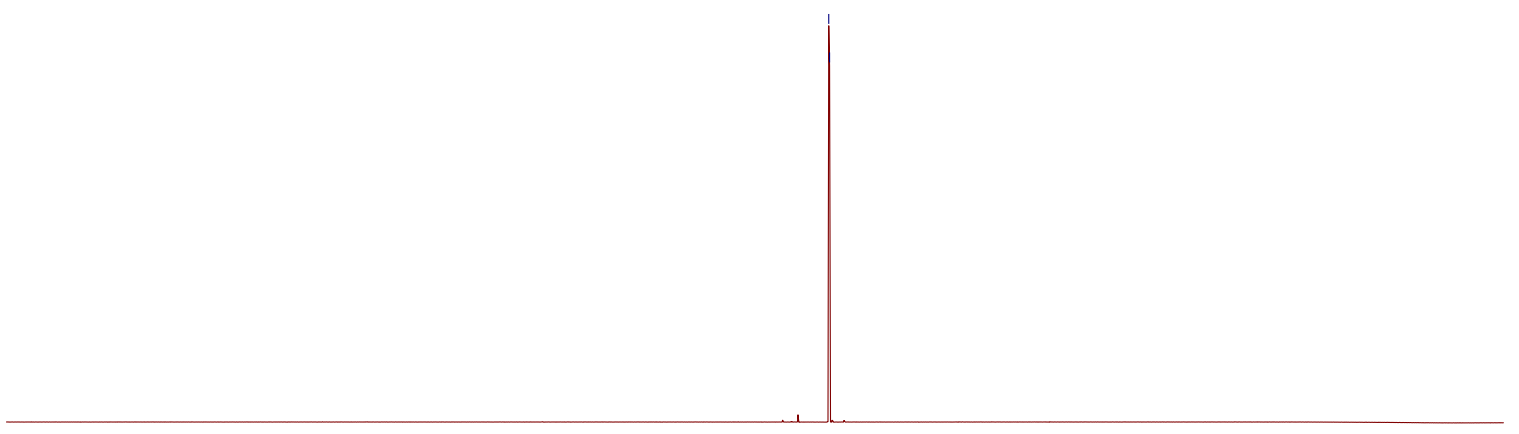

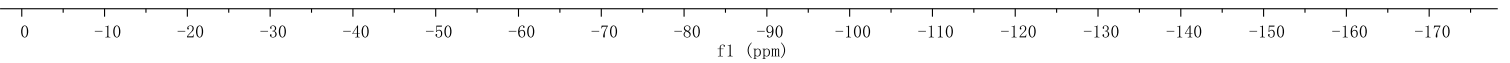


${ }^{1} \mathrm{H}$ NMR spectrum of (Z)-3-((difluoromethyl)thio)-2-(naphthalen-2-yl)-N-(quinolin-8-yl)acrylamide (3o)

$600 \mathrm{MHz}, \mathrm{CDCl}_{3}, 23{ }^{\circ} \mathrm{C}$

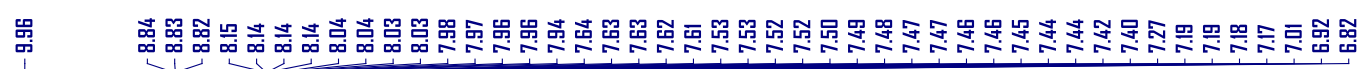

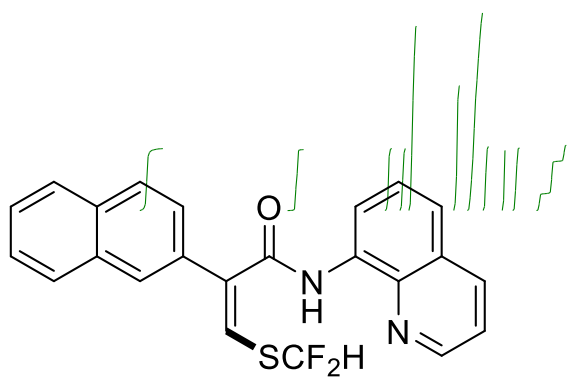

30

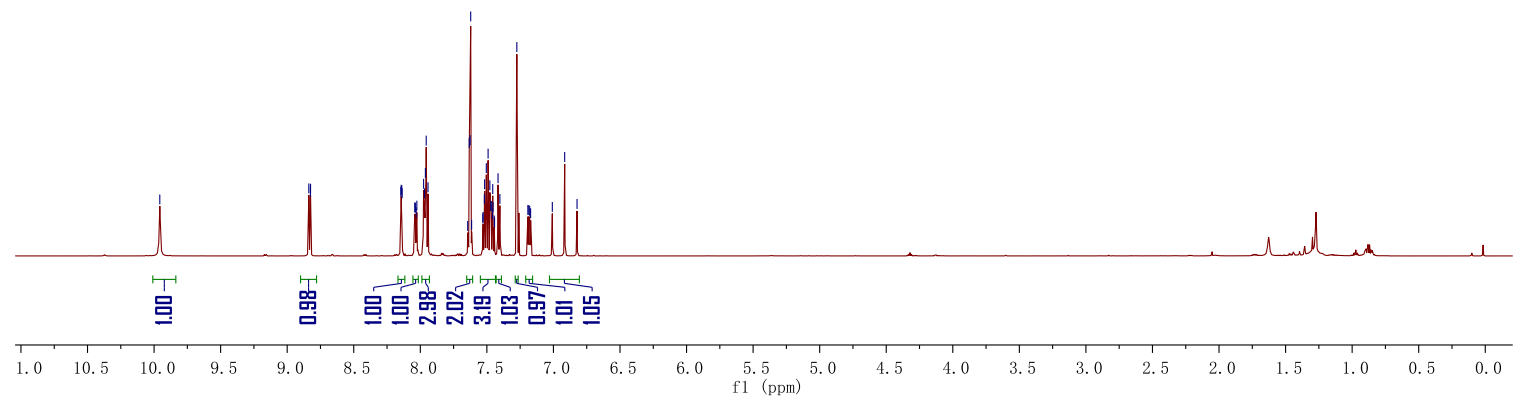

${ }^{13} \mathrm{C}\left\{{ }^{1} \mathrm{H}\right\}$ NMR spectrum of (Z)-3-((difluoromethyl)thio)-2-(naphthalen-2-yl)-N-(quinolin-8-yl)acrylamide (3o) $151 \mathrm{MHz}, \mathrm{CDCl}_{3}, 23{ }^{\circ} \mathrm{C}$

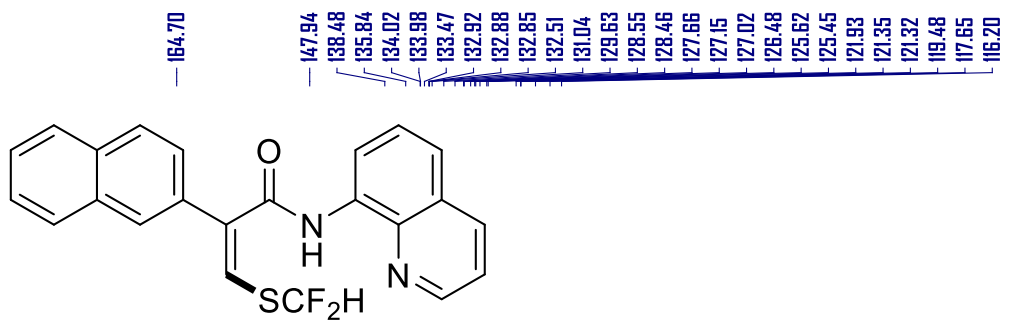

30

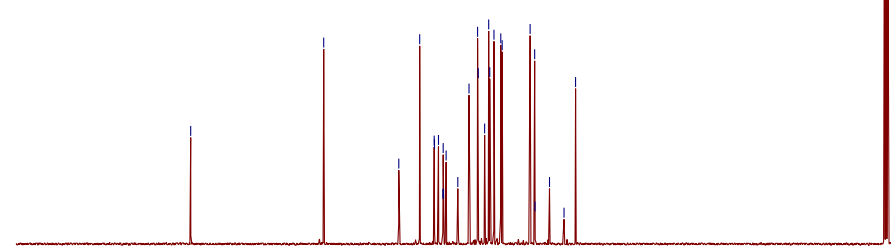

$180 \quad 170 \quad 160$

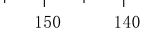

120

110

$100 \quad \begin{array}{r}90 \\ \mathrm{f} 1 \quad(\mathrm{ppm})\end{array}$ 
${ }^{19}$ F NMR spectrum of (Z)-3-((difluoromethyl)thio)-2-(naphthalen-2-yl)-N-(quinolin-8-yl)acrylamide (3o) $565 \mathrm{MHz}, \mathrm{CDCl}_{3}, 23{ }^{\circ} \mathrm{C}$

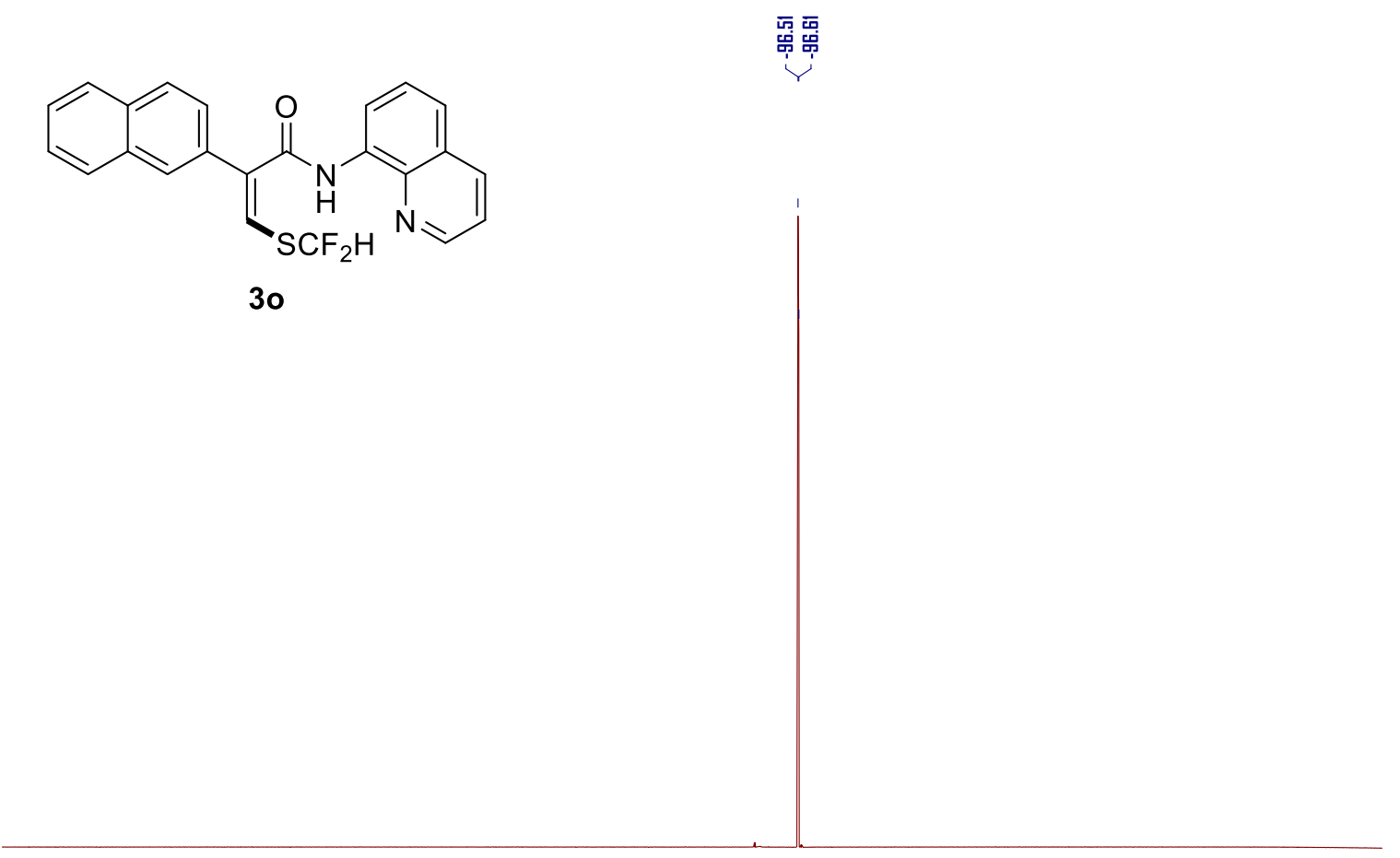

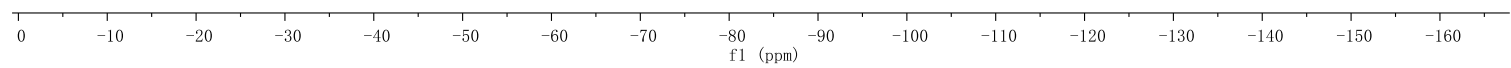

${ }^{1} \mathrm{H}$ NMR spectrum of (Z)-3-((difluoromethyl)thio)-2-methyl-N-(quinolin-8-yl)acrylamide (3p) $600 \mathrm{MHz}, \mathrm{CDCl}_{3}, 23{ }^{\circ} \mathrm{C}$

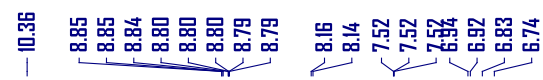

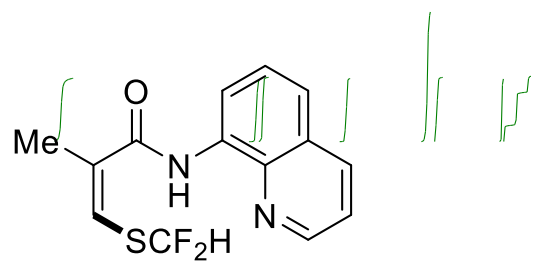

$3 p$

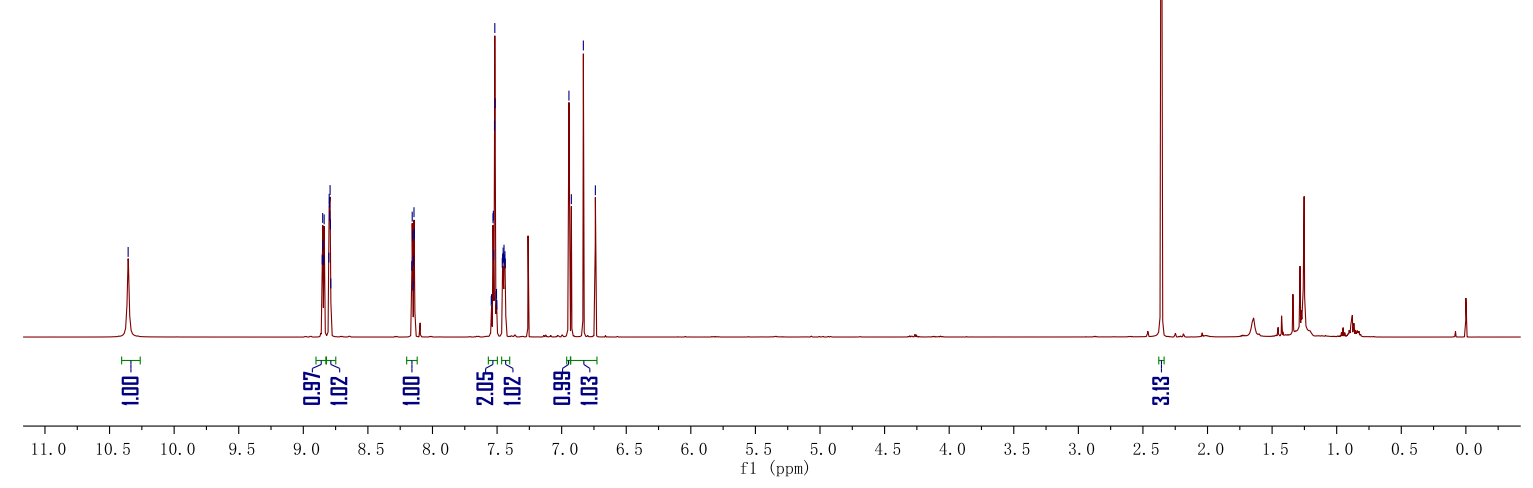


${ }^{13} \mathrm{C}\left\{{ }^{1} \mathrm{H}\right\}$ NMR spectrum of (Z)-3-((difluoromethyl)thio)-2-methyl-N-(quinolin-8-yl)acrylamide (3p) $151 \mathrm{MHz}, \mathrm{CDCl}_{3}, 23{ }^{\circ} \mathrm{C}$

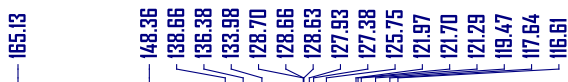<smiles>Cc1cccc(F)c1NC(=O)/C(=C\C(F)(F)F)Nc1cccc2cccnc12</smiles>

$3 p$
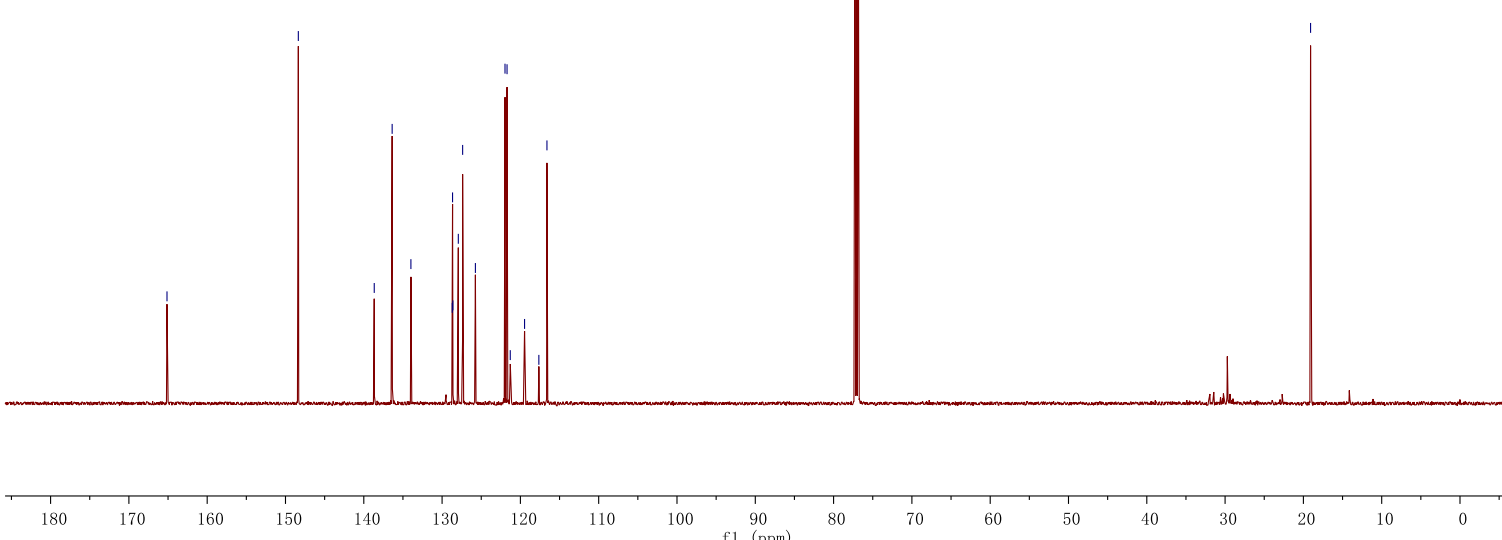

${ }^{19} \mathrm{~F}$ NMR spectrum of (Z)-3-((difluoromethyl)thio)-2-methyl-N-(quinolin-8-yl)acrylamide (3p) $565 \mathrm{MHz}, \mathrm{CDCl}_{3}, 23^{\circ} \mathrm{C}$
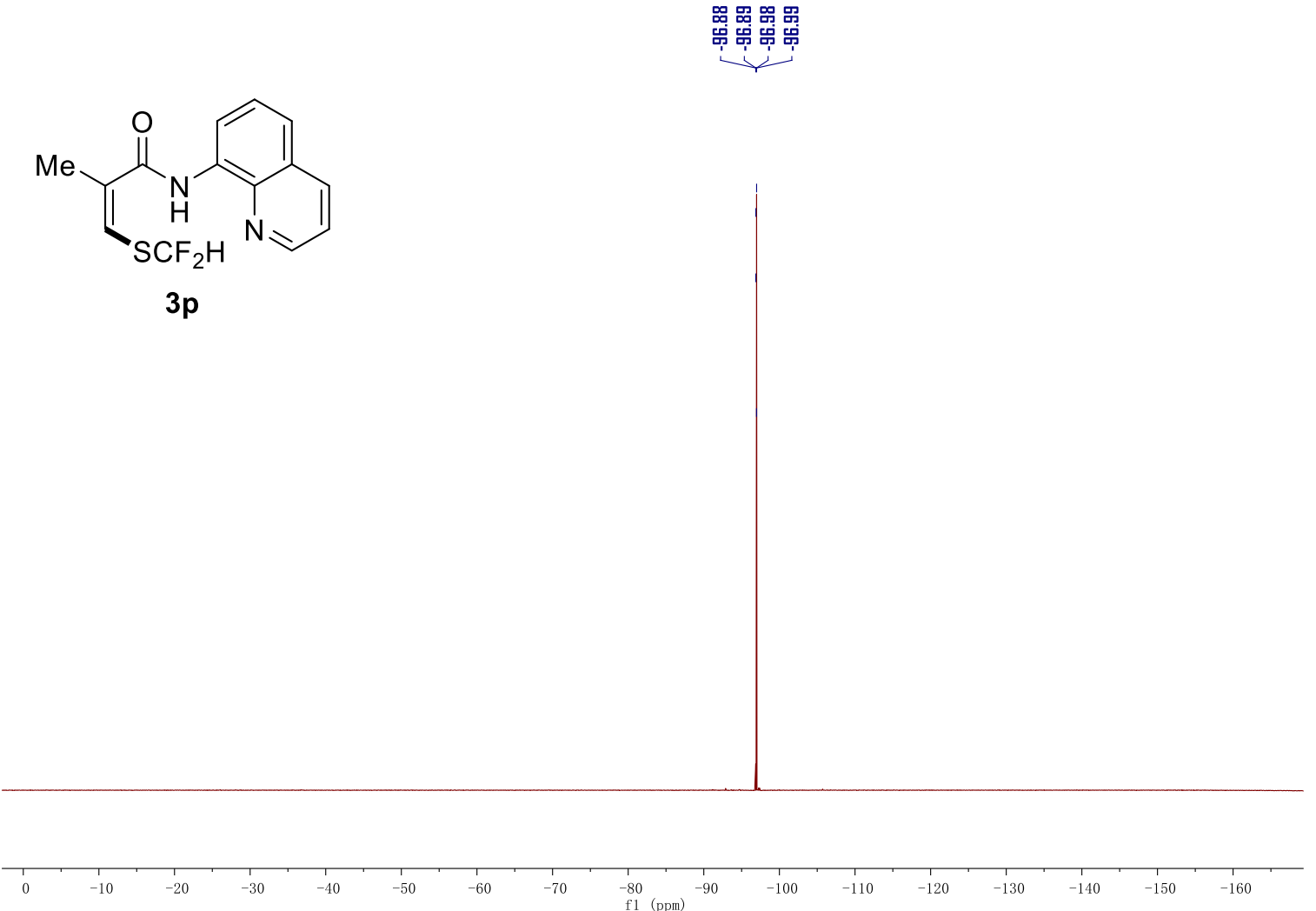
${ }^{1} \mathrm{H}$ NMR spectrum of (Z)-2-benzyl-3-((difluoromethyl)thio)-N-(quinolin-8-yl)acrylamide (3q) $600 \mathrm{MHz}, \mathrm{CDCl}_{3}, 23{ }^{\circ} \mathrm{C}$

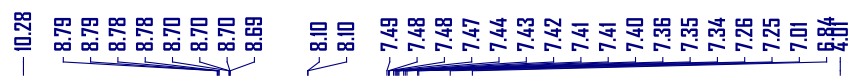<smiles>C=CC(=O)C(=C[Sn](F)(F)F)C(=O)Nc1cccc2cccnc12</smiles>

$3 q$

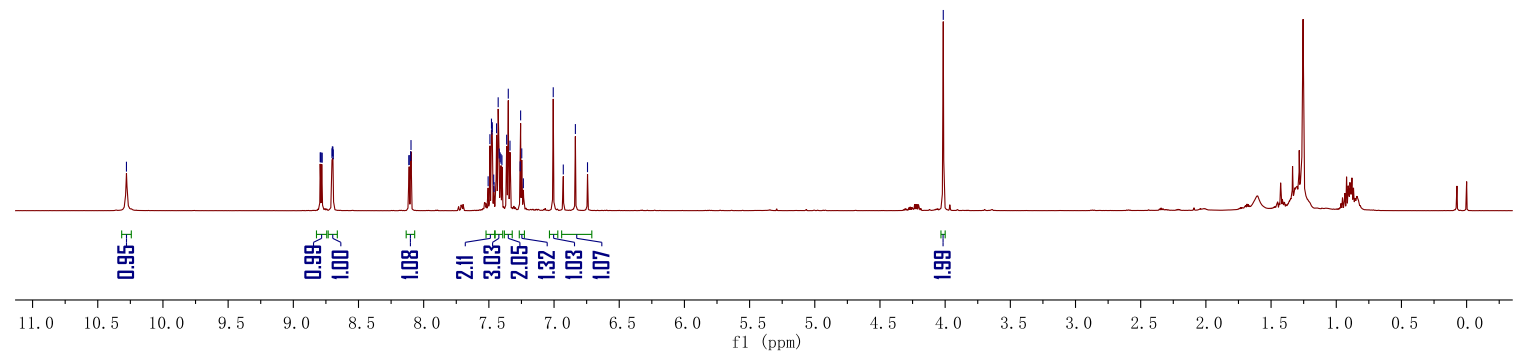

${ }^{13} \mathrm{C}\left\{{ }^{1} \mathrm{H}\right\}$ NMR spectrum of (Z)-2-benzyl-3-((difluoromethyl)thio)- $N$-(quinolin-8-yl)acrylamide (3q) $151 \mathrm{MHz}, \mathrm{CDCl}_{3}, 23{ }^{\circ} \mathrm{C}$

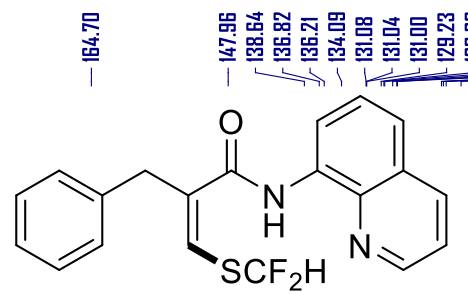

$3 q$

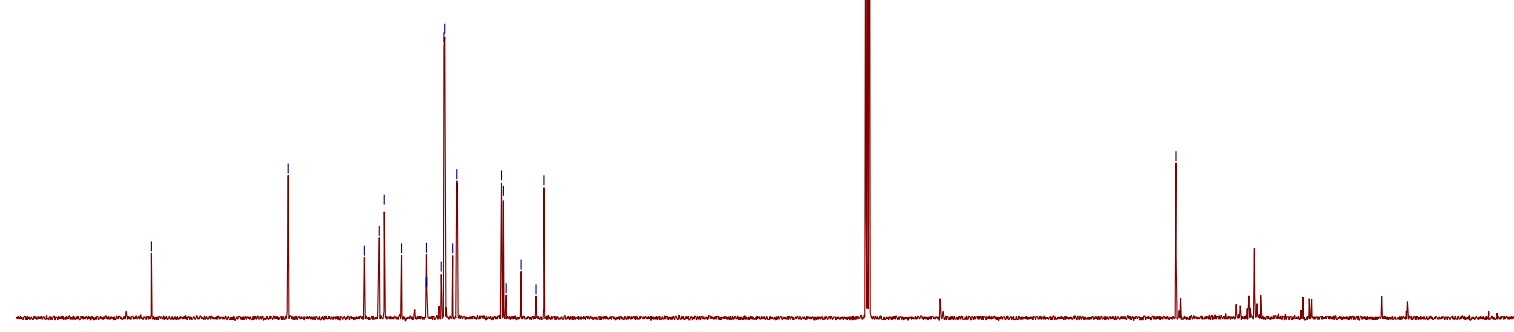

18
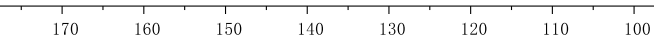

90 
${ }^{19} \mathrm{~F}$ NMR spectrum of (Z)-2-benzyl-3-((difluoromethyl)thio)-N-(quinolin-8-yl)acrylamide (3q) $565 \mathrm{MHz}, \mathrm{CDCl}_{3}, 23{ }^{\circ} \mathrm{C}$<smiles>CCS/C=C(/Cc1ccccc1)C(=O)Nc1cccc2cccnc12</smiles>

$3 q$

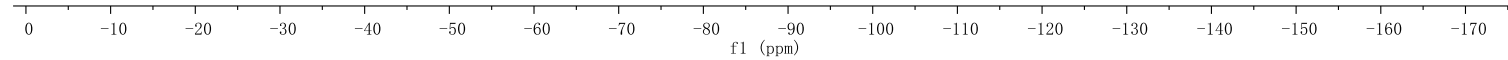

${ }^{1} \mathrm{H}$ NMR spectrum of (Z)-2-(((difluoromethyl)thio)methylene)- $N$-(quinolin-8-yl)pent-4-enamide (3r) $600 \mathrm{MHz}, \mathrm{CDCl}_{3}, 23{ }^{\circ} \mathrm{C}$

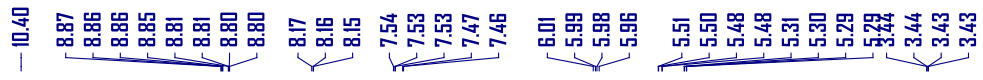

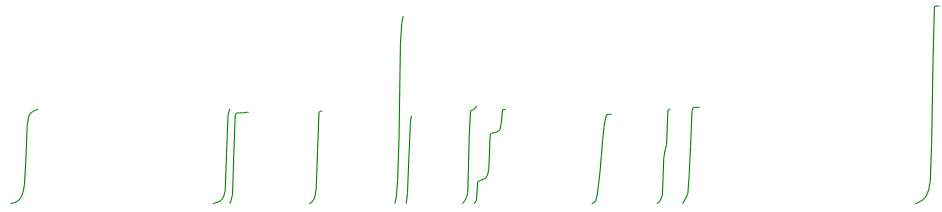<smiles>C=CC/C(=C/SCC)C(=O)Nc1cccc2cccnc12</smiles>

$3 \mathbf{r}$

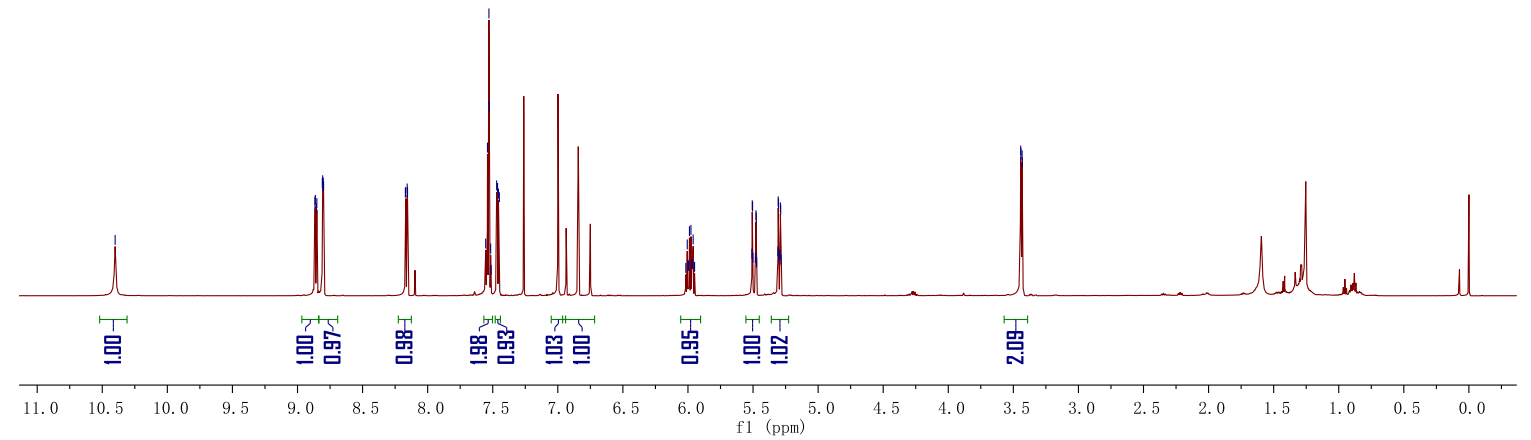


${ }^{13} \mathrm{C}\left\{{ }^{1} \mathrm{H}\right\}$ NMR spectrum of (Z)-2-(((difluoromethyl)thio)methylene)- $N$-(quinolin-8-yl)pent-4-enamide (3r) $151 \mathrm{MHz}, \mathrm{CDCl}_{3}, 23{ }^{\circ} \mathrm{C}$
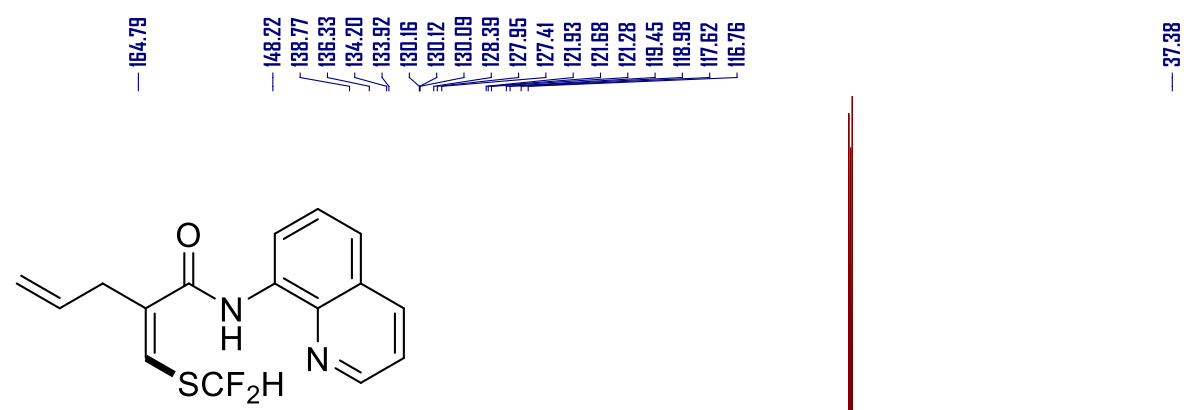

$3 r$
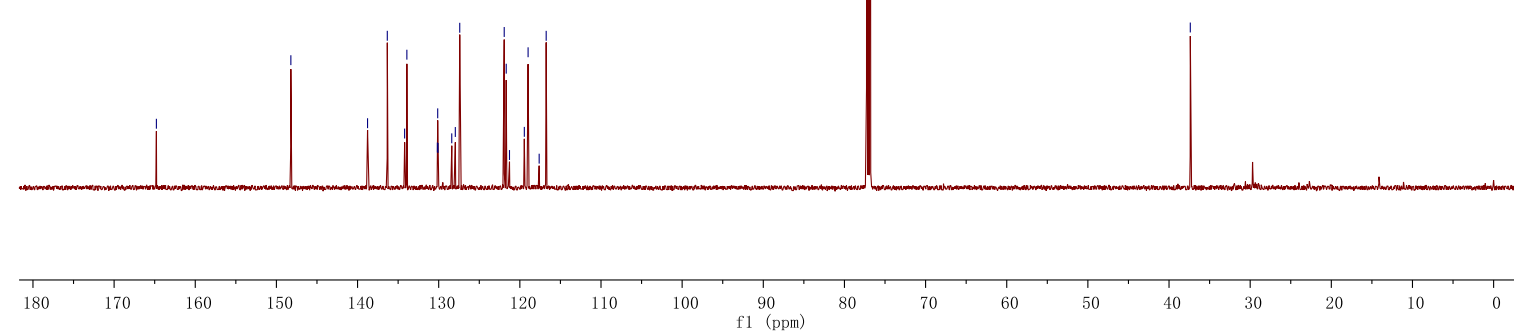

${ }^{19} \mathrm{~F}$ NMR spectrum of (Z)-2-(((difluoromethyl)thio)methylene)-N-(quinolin-8-yl)pent-4-enamide (3r) $565 \mathrm{MHz}, \mathrm{CDCl}_{3}, 23{ }^{\circ} \mathrm{C}$<smiles>C=CC/C(=C/SCC)C(=O)Nc1cccc2cccnc12</smiles>

$3 r$ 
${ }^{1} \mathrm{H}$ NMR spectrum of (Z)-3-((difluoromethyl)thio)-2-(ethoxymethyl)-N-(quinolin-8-yl)acrylamide (3s) $600 \mathrm{MHz}, \mathrm{CDCl}_{3}, 23{ }^{\circ} \mathrm{C}$

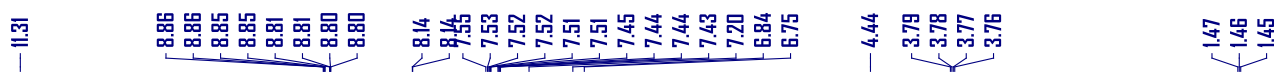

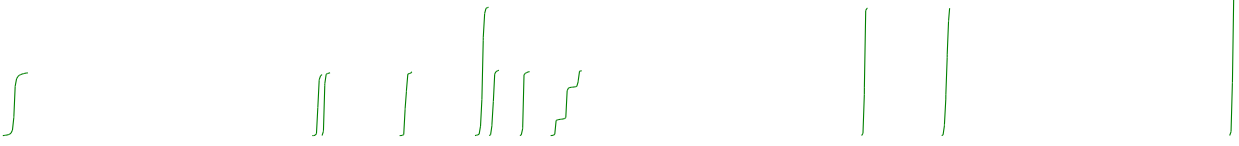<smiles>CCOC/C(=C/SC(F)(F)F)C(=O)Nc1cccc2cccnc12</smiles>

3s

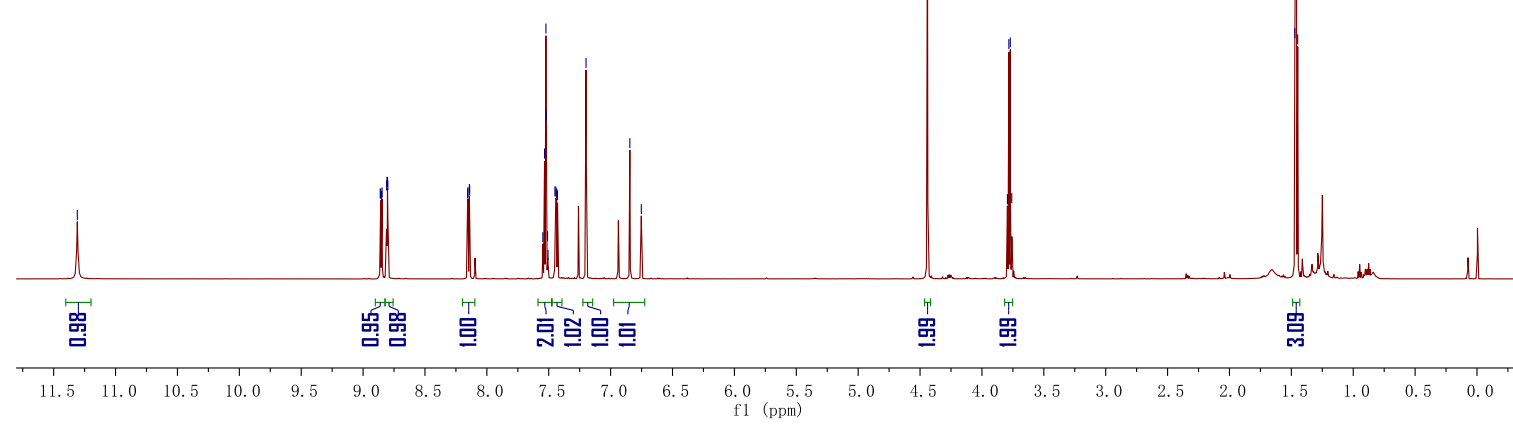

${ }^{13} \mathrm{C}\left\{{ }^{1} \mathrm{H}\right\}$ NMR spectrum of (Z)-3-((difluoromethyl)thio)-2-(ethoxymethyl)- $N$-(quinolin-8-yl)acrylamide (3s) $151 \mathrm{MHz}, \mathrm{CDCl}_{3}, 23{ }^{\circ} \mathrm{C}$

\begin{tabular}{|c|c|}
\hline 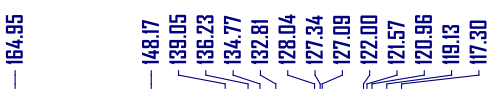 & 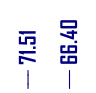 \\
\hline
\end{tabular}<smiles>CCOCC(=C[C-](F)F)C(=O)Nc1cccc2cccnc12</smiles>

3s
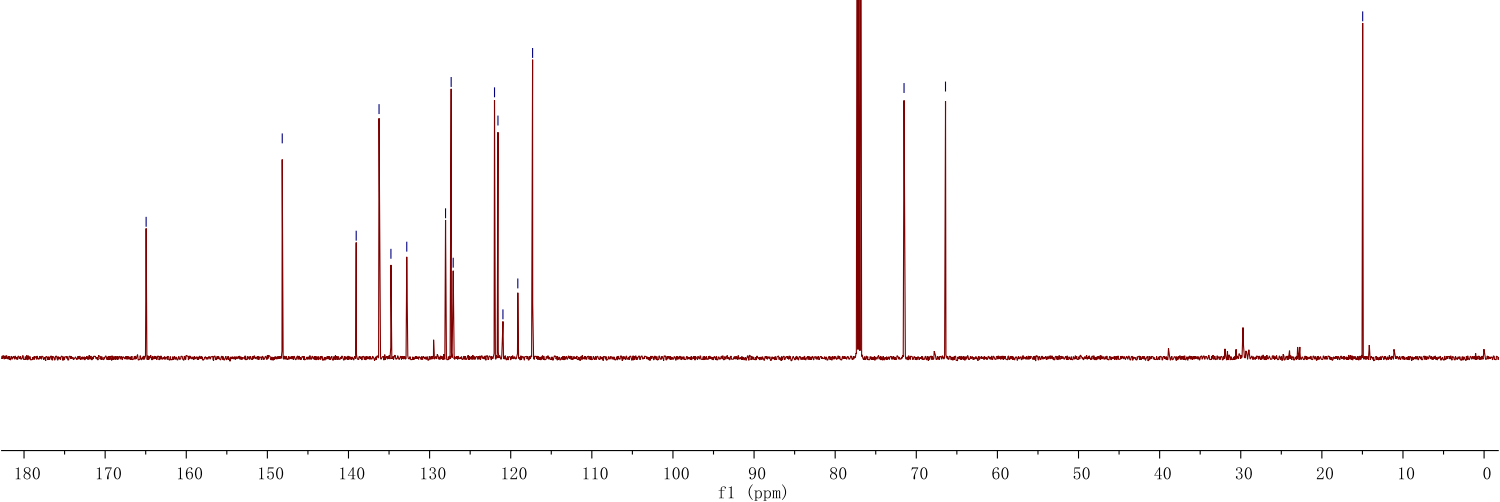
${ }^{19}$ F NMR spectrum of (Z)-3-((difluoromethyl)thio)-2-(ethoxymethyl)-N-(quinolin-8-yl)acrylamide (3s) $565 \mathrm{MHz}, \mathrm{CDCl}_{3}, 23{ }^{\circ} \mathrm{C}$

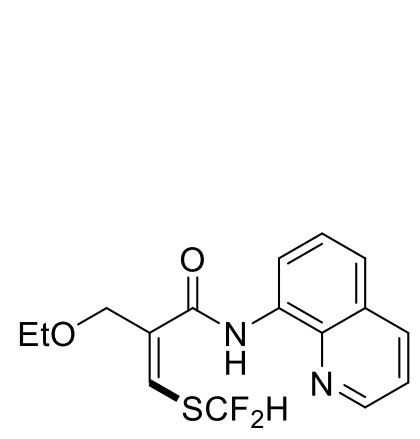

$3 s$

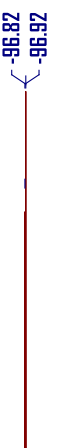

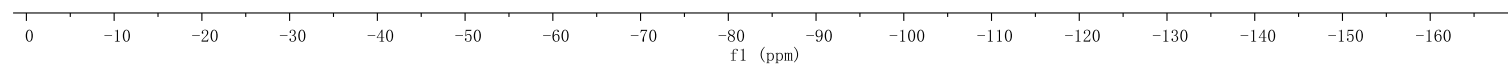

${ }^{1} \mathrm{H}$ NMR spectrum of (Z)-3-((difluoromethyl)thio)-2-methyl-3-phenyl-N-(quinolin-8-yl)acrylamide (3t) $600 \mathrm{MHz}, \mathrm{CDCl}_{3}, 23{ }^{\circ} \mathrm{C}$
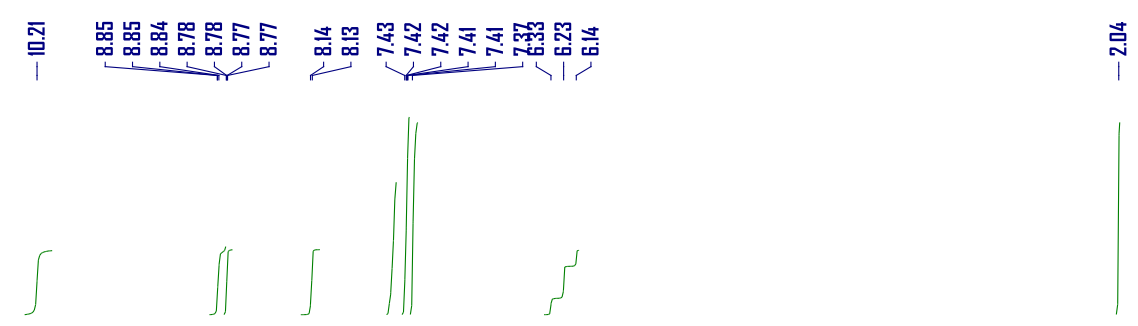<smiles>CC(F)=C(C(C)=C(C(=O)Nc1cccc2cccnc12)c1ccccc1)c1ccccc1</smiles>

$3 \mathbf{t}$

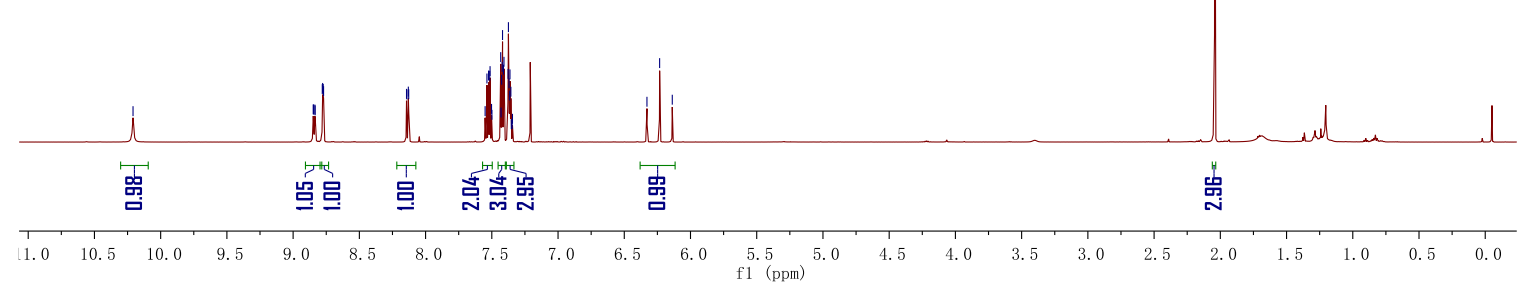


${ }^{13} \mathrm{C}\left\{{ }^{1} \mathrm{H}\right\}$ NMR spectrum of (Z)-3-((difluoromethyl)thio)-2-methyl-3-phenyl-N-(quinolin-8-yl)acrylamide (3t) $151 \mathrm{MHz}, \mathrm{CDCl}_{3}, 23{ }^{\circ} \mathrm{C}$

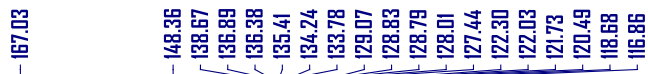<smiles>C/C(C(=O)Nc1cccc2cccnc12)=C(\c1ccccc1)C(F)(F)F</smiles>

$3 t$

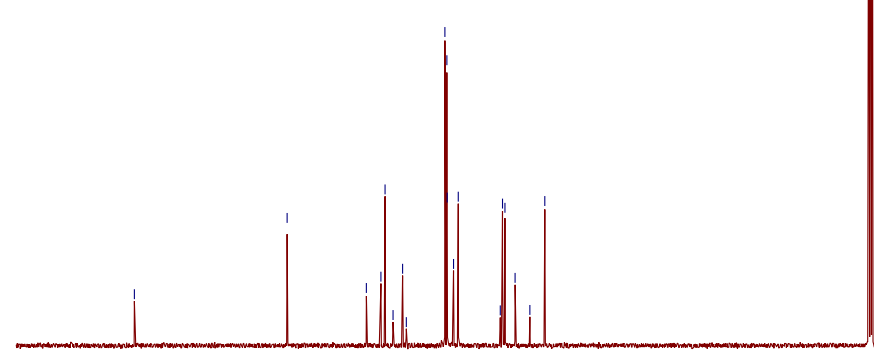

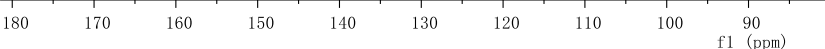

${ }^{19} \mathrm{~F}$ NMR spectrum of (Z)-3-((difluoromethyl)thio)-2-methyl-3-phenyl- $N$-(quinolin-8-yl)acrylamide (3t) $565 \mathrm{MHz}, \mathrm{CDCl}_{3}, 23{ }^{\circ} \mathrm{C}$

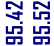<smiles>C/C(C(=O)Nc1cccc2cccnc12)=C(/c1ccccc1)C(F)F</smiles>

$3 t$

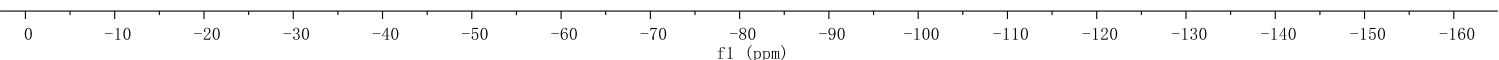


${ }^{1} \mathrm{H}$ NMR spectrum of (Z)-3-((difluoromethyl)thio)-2-methyl-N-(quinolin-8-yl)but-2-enamide (3u) $600 \mathrm{MHz}, \mathrm{CDCl}_{3}, 23{ }^{\circ} \mathrm{C}$<smiles>CCC(C)=C(C)C(=O)Nc1cccc2cccnc12</smiles>

3u

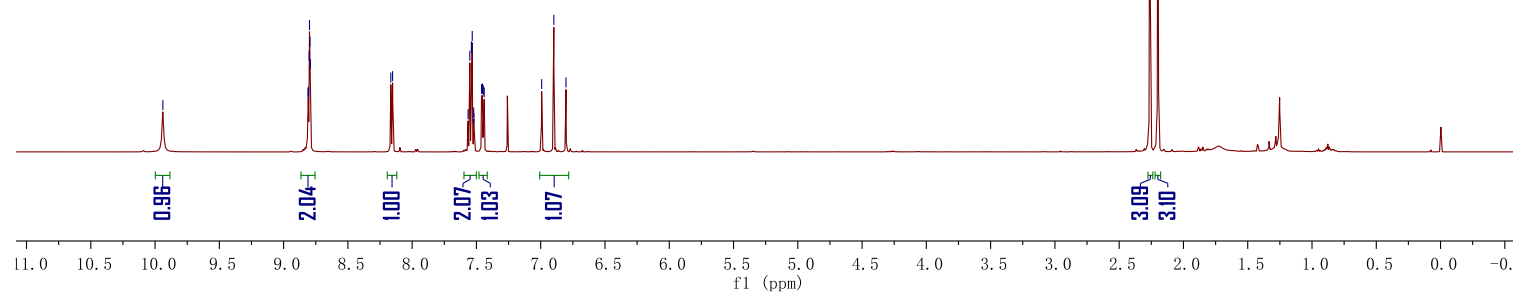

${ }^{13} \mathrm{C}\left\{{ }^{1} \mathrm{H}\right\}$ NMR spectrum of (Z)-3-((difluoromethyl)thio)-2-methyl-N-(quinolin-8-yl)but-2-enamide (3u) $151 \mathrm{MHz}, \mathrm{CDCl}_{3}, 23{ }^{\circ} \mathrm{C}$

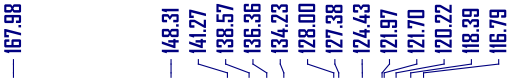<smiles>C/C(C(=O)Nc1cccc2cccnc12)=C(\C)C(F)(F)F</smiles>

3u
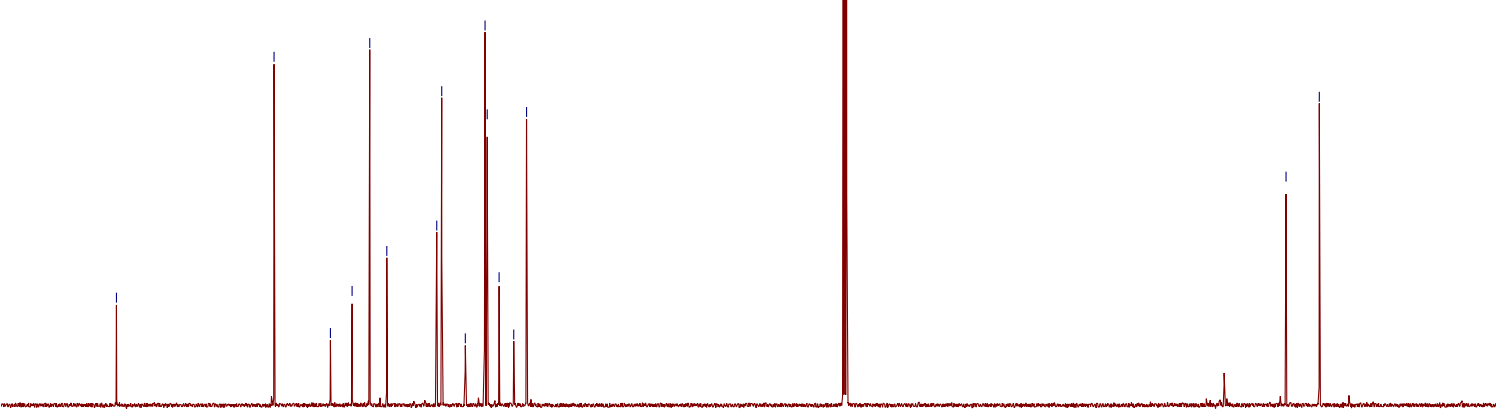
${ }^{19} \mathrm{~F}$ NMR spectrum of (Z)-3-((difluoromethyl)thio)-2-methyl-N-(quinolin-8-yl)but-2-enamide (3u) $565 \mathrm{MHz}, \mathrm{CDCl}_{3}, 23{ }^{\circ} \mathrm{C}$<smiles>CC(C(=O)Nc1cccc2cccnc12)=C(C)C(F)(F)F</smiles>

$3 \mathbf{u}$

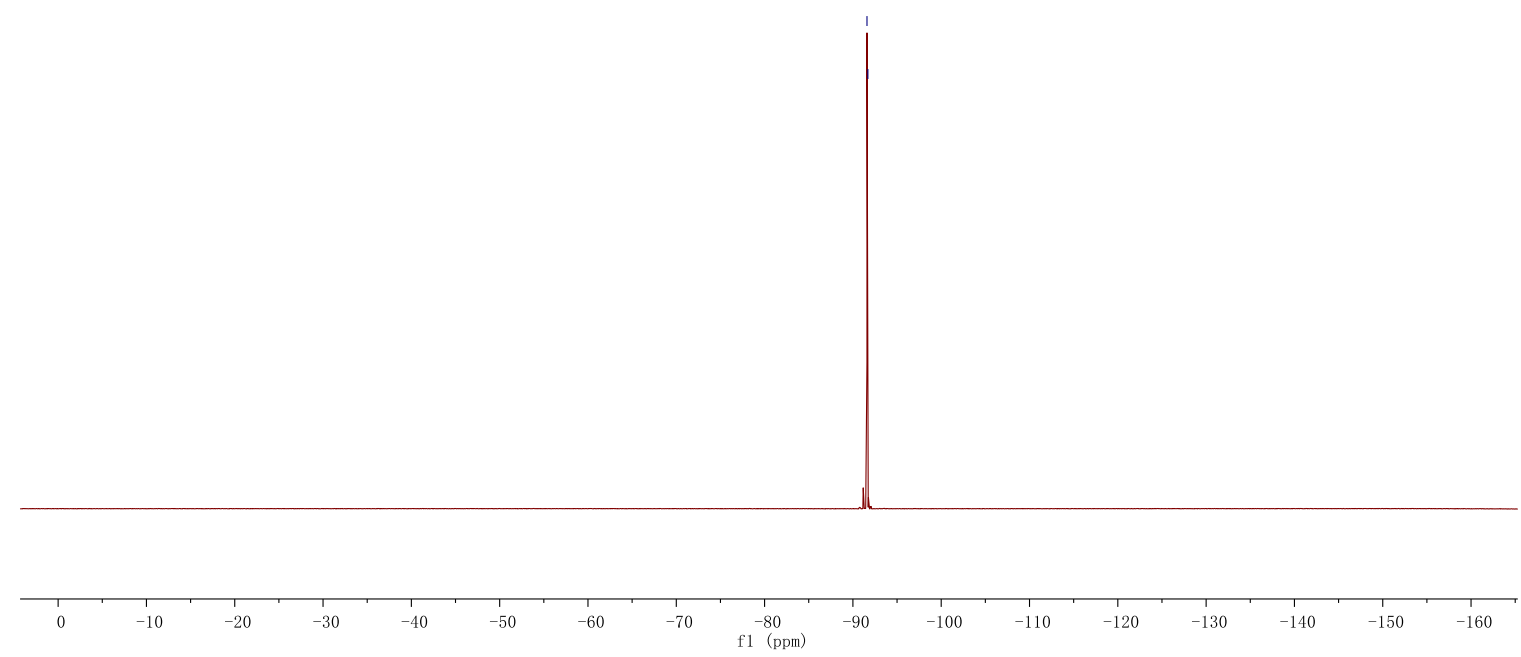

${ }^{1} H$ NMR spectrum of (Z)-3-((difluoromethyl)thio)-3-phenyl-N-(quinolin-8-yl)acrylamide (3v) $600 \mathrm{MHz}, \mathrm{CDCl}_{3}, 23{ }^{\circ} \mathrm{C}$

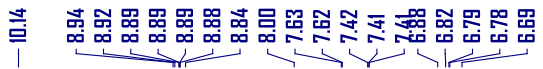<smiles>CCCC(F)=CC(=O)Nc1cccc2cccnc12</smiles>

3v

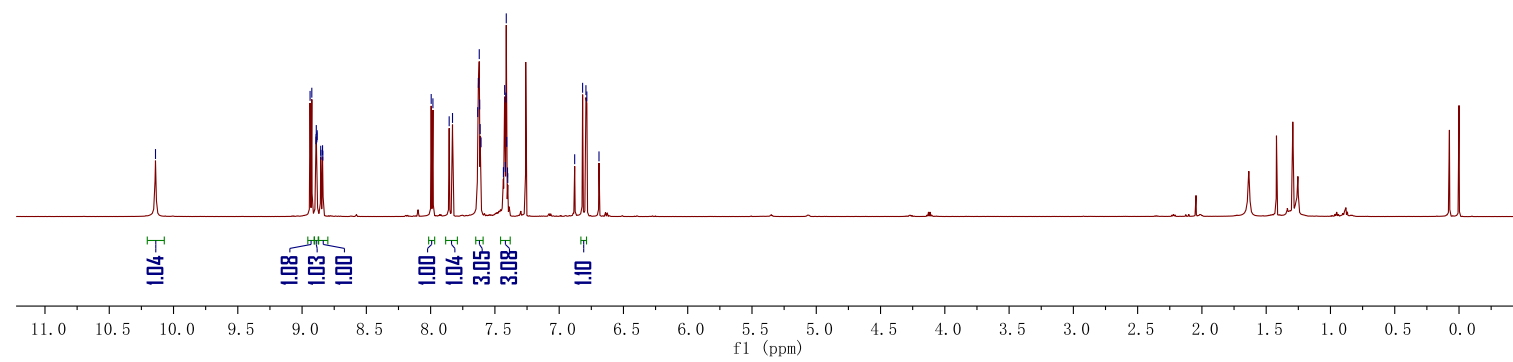


${ }^{13} \mathrm{C}\left\{{ }^{1} \mathrm{H}\right\}$ NMR spectrum of (Z)-3-((difluoromethyl)thio)-3-phenyl-N-(quinolin-8-yl)acrylamide (3v) $151 \mathrm{MHz}, \mathrm{CDCl}_{3}, 23{ }^{\circ} \mathrm{C}$

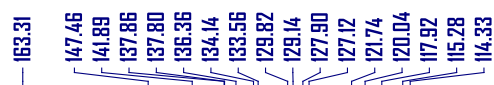<smiles>O=C(/C=C(\SC(F)(F)F)c1ccccc1)Nc1cccc2cccnc12</smiles>

$3 v$

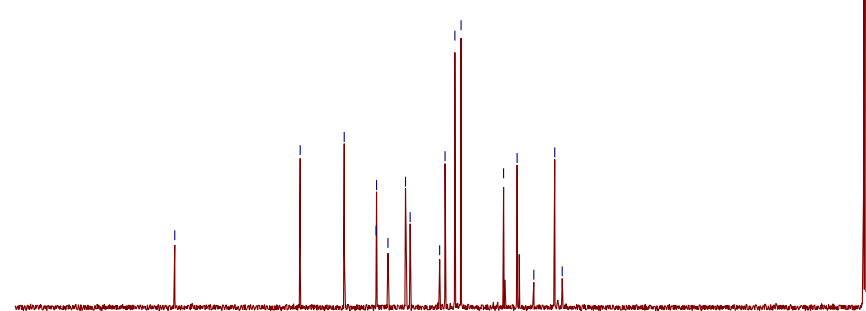

$180 \quad 170 \quad 160$

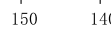

(90 90

${ }^{19} \mathrm{~F}$ NMR spectrum of (Z)-3-((difluoromethyl)thio)-3-phenyl-N-(quinolin-8-yl)acrylamide (3v) $565 \mathrm{MHz}^{\mathrm{CDCl}} 3,23^{\circ} \mathrm{C}$

놓ำ<smiles>O=C(/C=C(\[Se-])c1ccccc1)Nc1cccc2cccnc12</smiles>

$3 v$

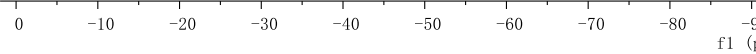


${ }^{1} \mathrm{H}$ NMR spectrum of (Z)-3-((difluoromethyl)thio)-3-ethoxy-N-(quinolin-8-yl)acrylamide (3w) $600 \mathrm{MHz}, \mathrm{CDCl}_{3}, 23{ }^{\circ} \mathrm{C}$<smiles>C=C1C=Nc2c(NC(=O)/C=C(/OCC)SCC)cccc21</smiles>

$3 w$

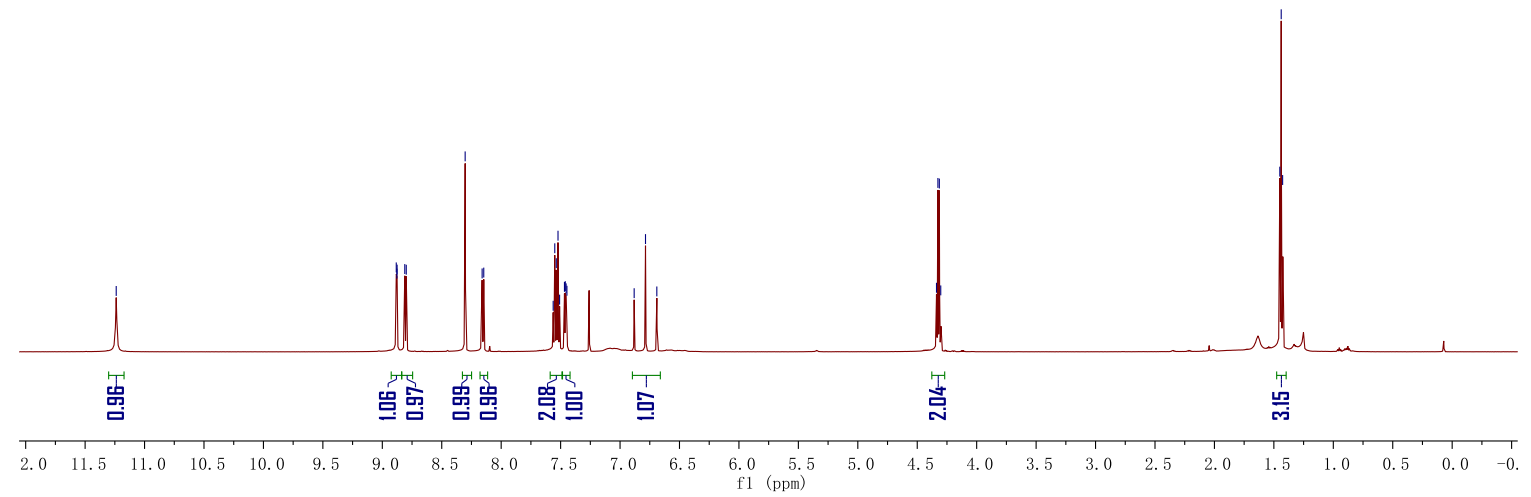

${ }^{13} \mathrm{C}\left\{{ }^{1} \mathrm{H}\right\}$ NMR spectrum of (Z)-3-((difluoromethyl)thio)-3-ethoxy-N-(quinolin-8-yl)acrylamide (3w) $151 \mathrm{MHz}, \mathrm{CDCl}_{3}, 23{ }^{\circ} \mathrm{C}$
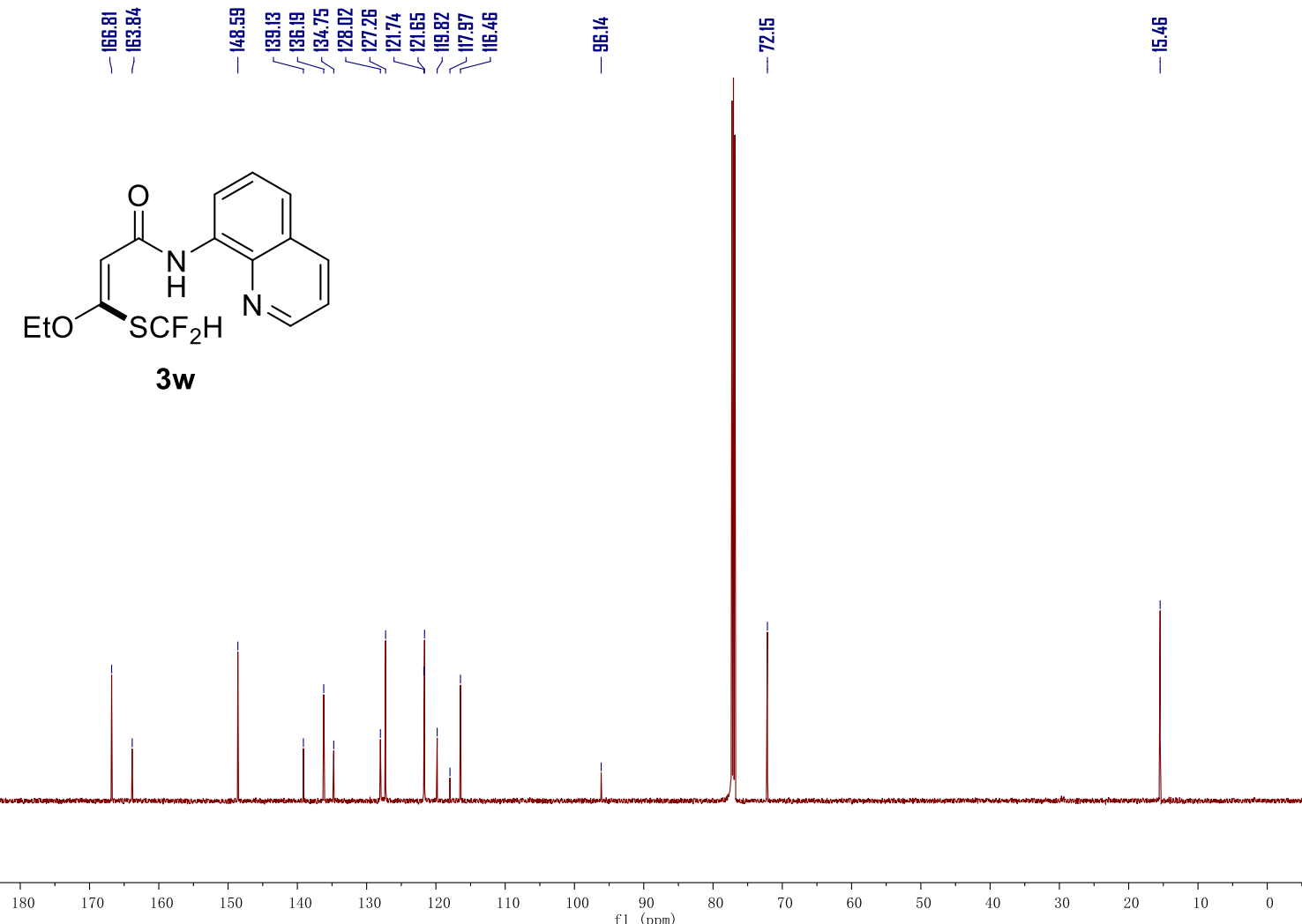
${ }^{19} \mathrm{~F}$ NMR spectrum of (Z)-3-((difluoromethyl)thio)-3-ethoxy-N-(quinolin-8-yl)acrylamide (3w) $565 \mathrm{MHz}, \mathrm{CDCl}_{3}, 23{ }^{\circ} \mathrm{C}$<smiles>CCOC(=CC(=O)Nc1cccc2cccnc12)C(F)(F)F</smiles>

$3 w$

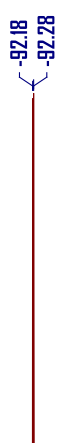

${ }^{1} \mathrm{H}$ NMR spectrum of 2-((difluoromethyl)thio)-N-(quinolin-8-yl)cyclohex-1-ene-1-carboxamide (3x) $600 \mathrm{MHz}, \mathrm{CDCl}_{3}, 23{ }^{\circ} \mathrm{C}$

算 计

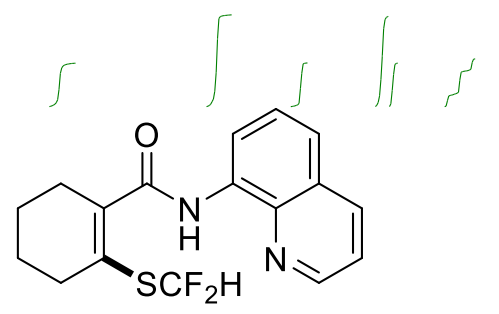

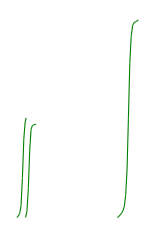

$3 \mathbf{x}$

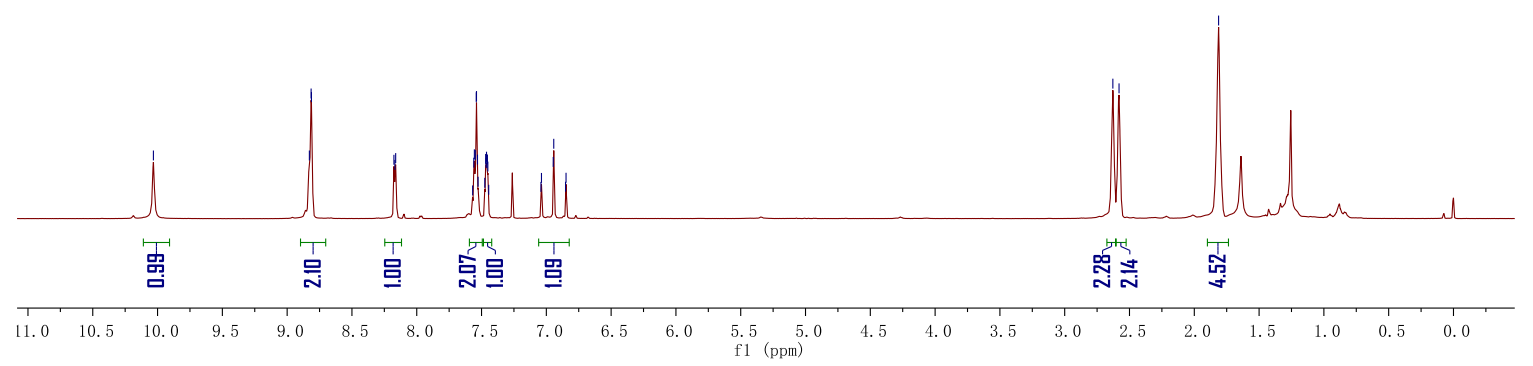


${ }^{13} \mathrm{C}\left\{{ }^{1} \mathrm{H}\right\}$ NMR spectrum of 2-((difluoromethyl)thio)-N-(quinolin-8-yl)cyclohex-1-ene-1-carboxamide (3x) $151 \mathrm{MHz}, \mathrm{CDCl}_{3}, 23{ }^{\circ} \mathrm{C}$

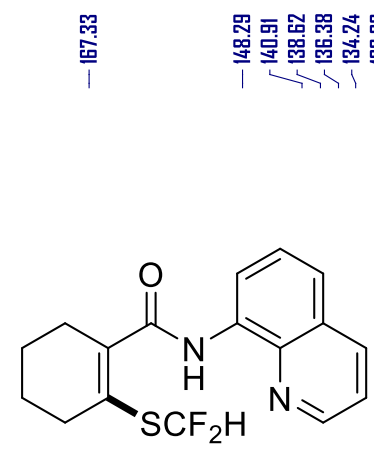

$3 \mathbf{x}$

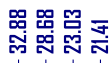

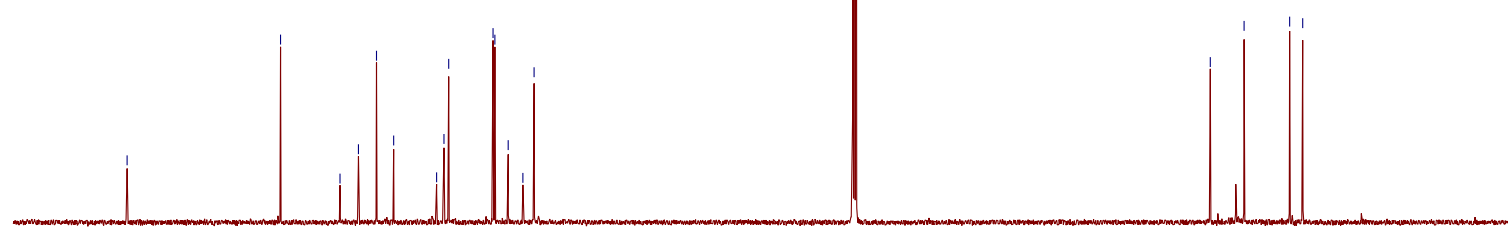

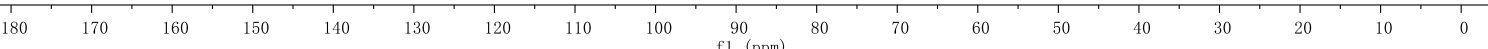

${ }^{19} \mathrm{~F}$ NMR spectrum of 2-((difluoromethyl)thio)-N-(quinolin-8-yl)cyclohex-1-ene-1-carboxamide (3x) $565 \mathrm{MHz}, \mathrm{CDCl}_{3}, 23{ }^{\circ} \mathrm{C}$<smiles>O=C(Nc1cccc2cccnc12)C1=C(SCC(F)(F)F)CCCC1</smiles>

$3 x$

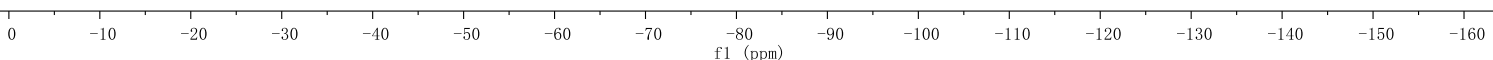


${ }^{1} \mathrm{H}$ NMR spectrum of (Z)-3-((difluoromethyl)sulfinyl)-2-phenyl-N-(quinolin-8-yl)acrylamide (4a')

$600 \mathrm{MHz}, \mathrm{CDCl}_{3}, 23{ }^{\circ} \mathrm{C}$

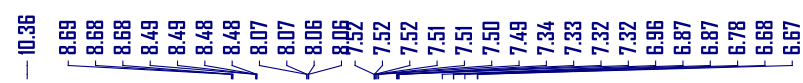<smiles>O=C(Nc1cccc2cccnc12)/C(=C\OC(F)(F)F)c1ccccc1</smiles>

$4 a^{\prime}$

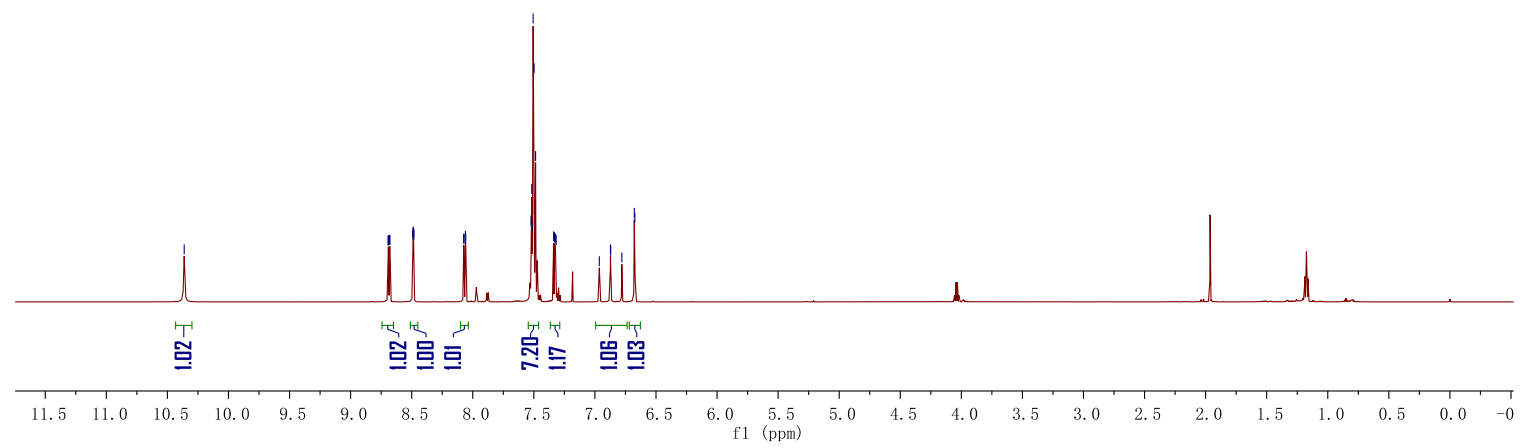

${ }^{13} \mathrm{C}$ NMR spectrum of (Z)-3-((difluoromethyl)sulfinyl)-2-phenyl-N-(quinolin-8-yl)acrylamide (4a') $151 \mathrm{MHz}, \mathrm{CDCl}_{3}, 23{ }^{\circ} \mathrm{C}$

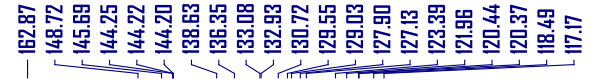<smiles>CCOC(=O)C=C(C(=O)Nc1cccc2c1N=CCC2)c1ccccc1</smiles>

4a'

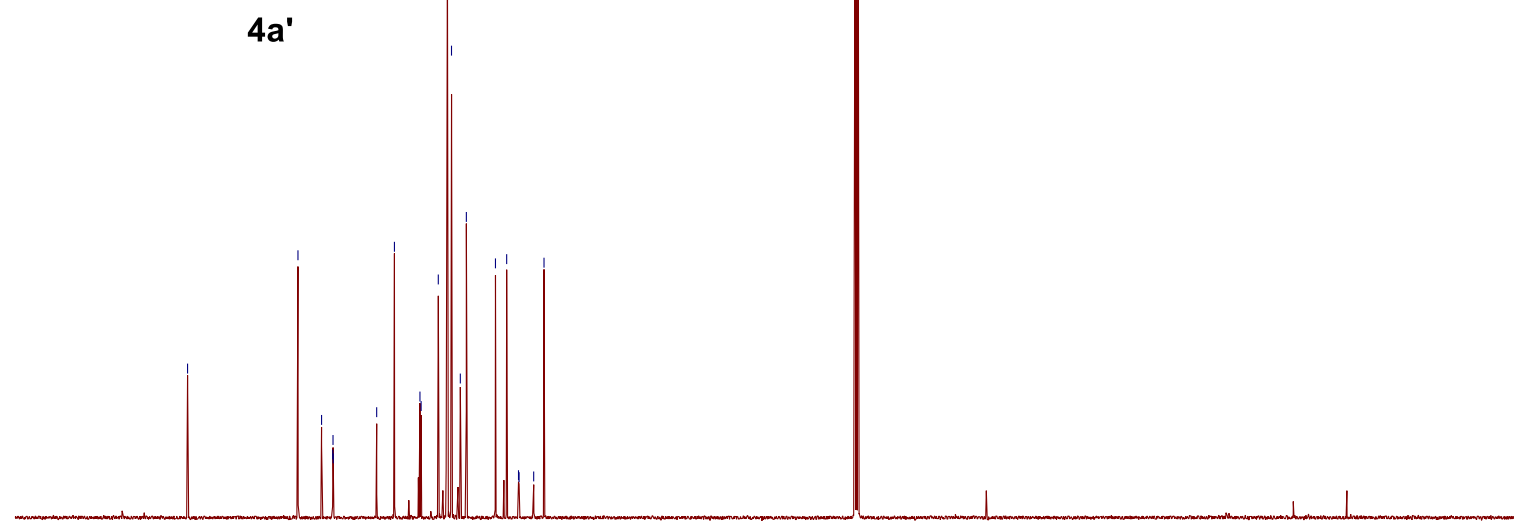


${ }^{19} \mathrm{~F}$ NMR spectrum of (Z)-3-((difluoromethyl)sulfinyl)-2-phenyl-N-(quinolin-8-yl)acrylamide (4a') $565 \mathrm{MHz}, \mathrm{CDCl}_{3}, 23{ }^{\circ} \mathrm{C}$

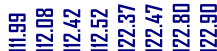<smiles>C=C(OCC)OCCOC(F)(F)C(=C(NC(=O)c1ccccc1)c1ccccn1)c1cccc2cccnc12</smiles>

$4 a^{\prime}$

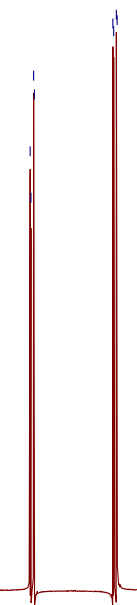

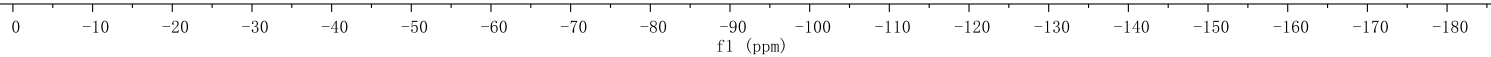

${ }^{1} \mathrm{H}$ NMR spectrum of (Z)-3-((difluoromethyl)sulfonyl)-2-phenyl-N-(quinolin-8-yl)acrylamide (4a") $600 \mathrm{MHz}, \mathrm{CDCl}_{3}, 23{ }^{\circ} \mathrm{C}$

量

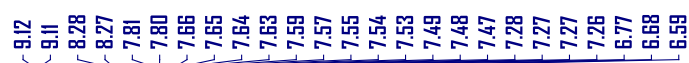

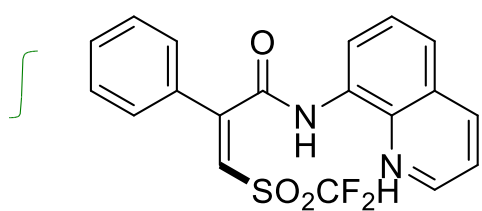

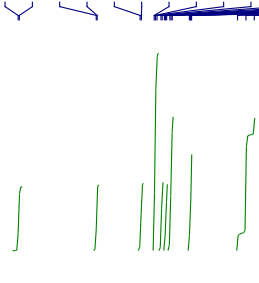

4a"

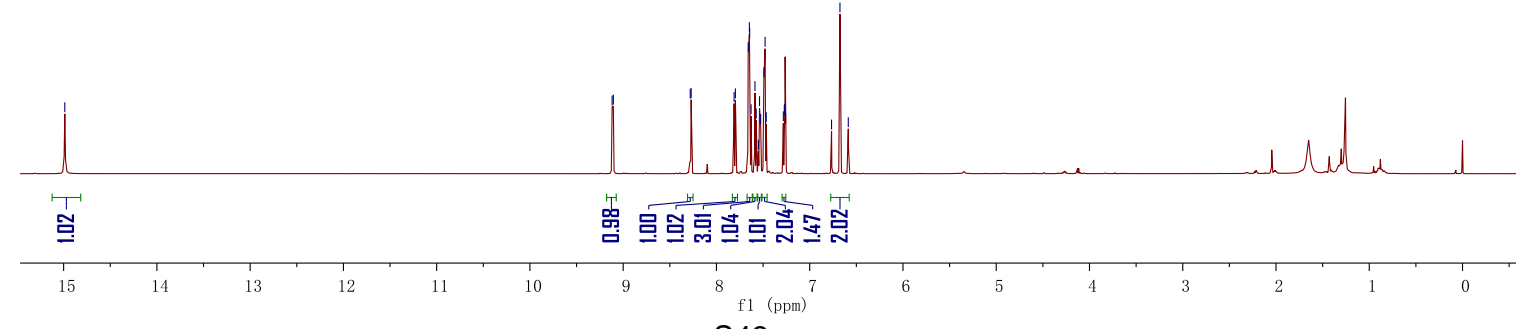


${ }^{13} \mathrm{C}$ NMR spectrum of (Z)-3-((difluoromethyl)sulfonyl)-2-phenyl-N-(quinolin-8-yl)acrylamide (4a") $151 \mathrm{MHz}, \mathrm{CDCl}_{3}, 23{ }^{\circ} \mathrm{C}$

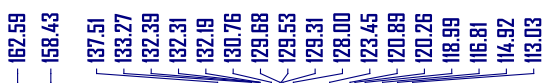<smiles>CCOC(=O)C=C(C(=O)Nc1cccc2cccnc12)c1ccccc1</smiles>

4a"

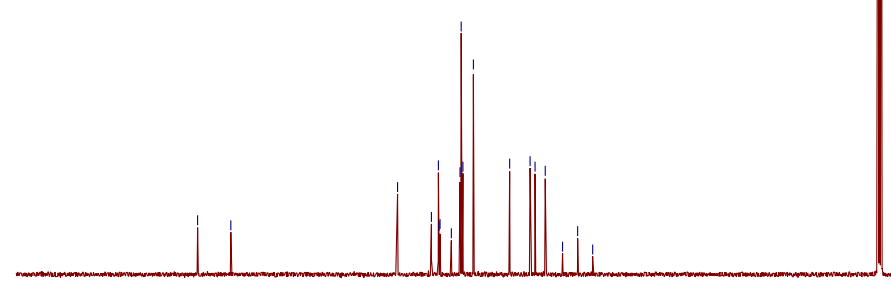

180

$170 \quad 160 \quad 150$

$140 \quad 130$

$120 \quad 110$

$100 \quad 90$

${ }^{19} \mathrm{~F}$ NMR spectrum of (Z)-3-((difluoromethyl)sulfonyl)-2-phenyl-N-(quinolin-8-yl)acrylamide (4a")

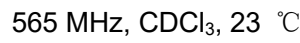<smiles>O=C(Nc1cccc2cccnc12)/C(=C\SOC(F)(F)F)c1ccccc1</smiles>

4a"

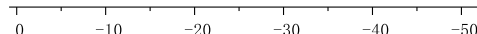


${ }^{1} \mathrm{H}$ NMR spectrum of methyl (Z)-3-((difluoromethyl)thio)-2-phenylacrylate (5a)

$600 \mathrm{MHz}, \mathrm{CDCl}_{3}, 23{ }^{\circ} \mathrm{C}$

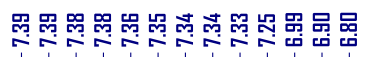<smiles>COC(=O)/C=C(\C(=O)OC)c1cccc(C)c1</smiles>

$5 a$

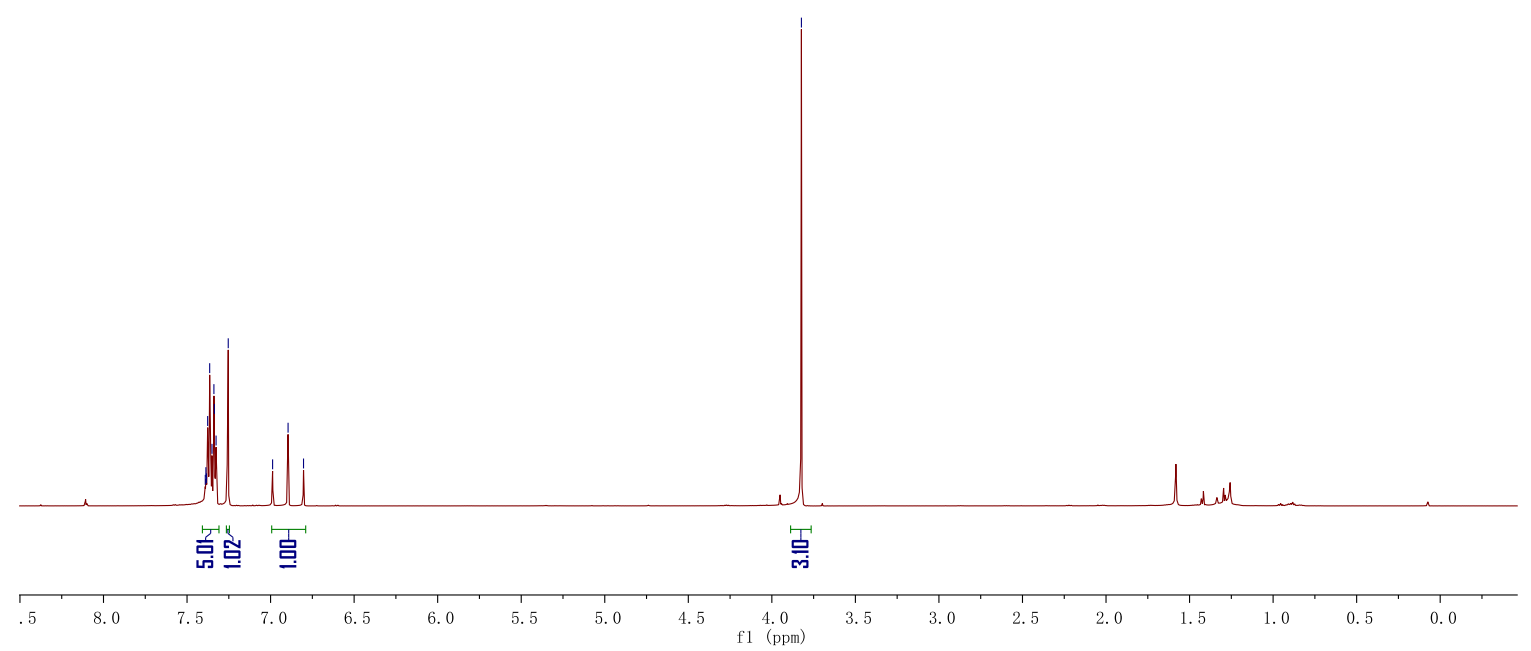

${ }^{13} \mathrm{C}$ NMR spectrum of methyl (Z)-3-((difluoromethyl)thio)-2-phenylacrylate (5a)

$151 \mathrm{MHz}, \mathrm{CDCl}_{3}, 23{ }^{\circ} \mathrm{C}$

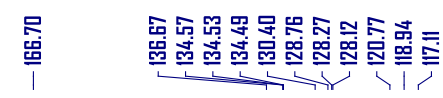<smiles>COC(=O)/C(=C\[S-]F)c1ccccc1</smiles>

$5 a$
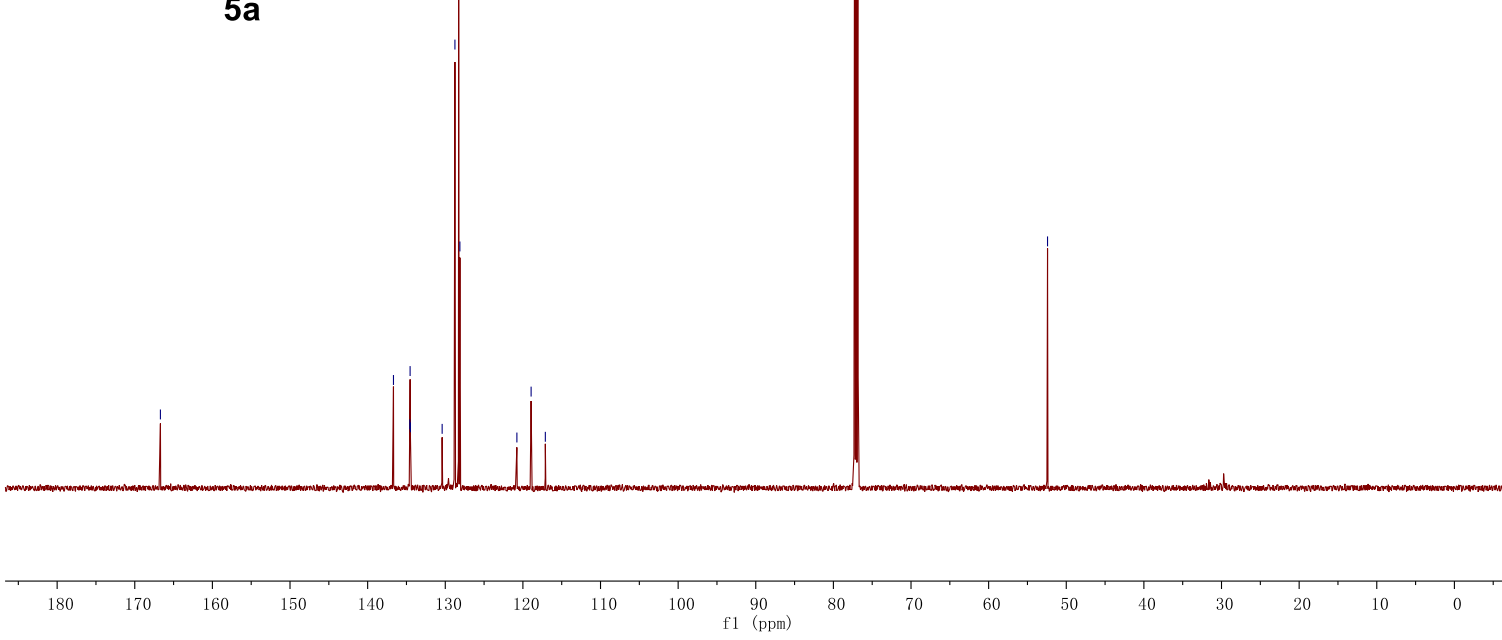
${ }^{19} \mathrm{~F}$ NMR spectrum of methyl (Z)-3-((difluoromethyl)thio)-2-phenylacrylate (5a)

$565 \mathrm{MHz}, \mathrm{CDCl}_{3}, 23{ }^{\circ} \mathrm{C}$

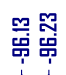

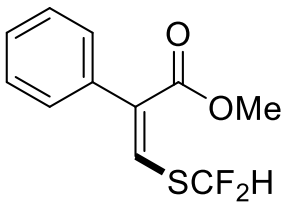

$5 a$

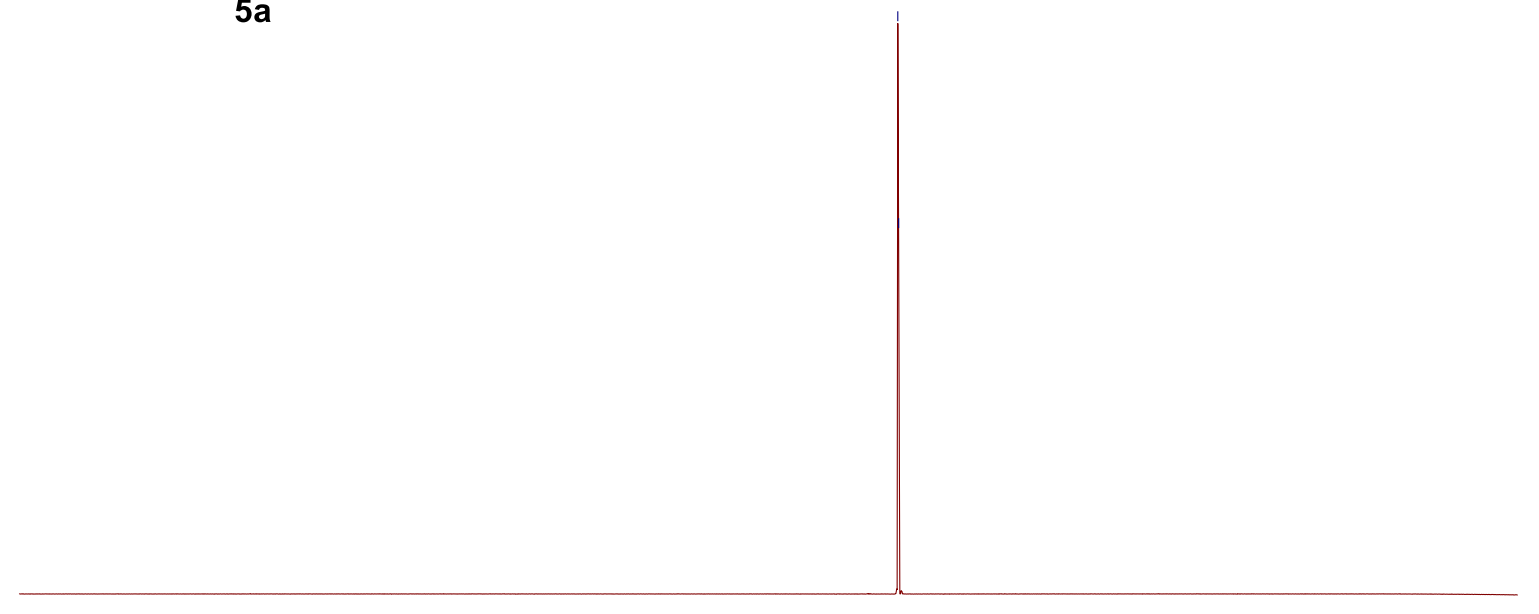

\begin{tabular}{rlllllllllllllll}
\hline & 1 & 1 & 1 & 1 & 1 & 1 & 1 & 1 & 1 & 1 & 1 & 1 & 1 & 1 & 1 \\
\hline & -20 & -30 & -40 & -50 & -60 & -70 & -80 & -90 & -100 & -110 & -120 & -130 & -140 & -150 & -160 \\
\hline
\end{tabular}

${ }^{1} \mathrm{H}$ NMR spectrum of (Z)-3-((difluoromethyl)thio)-2-phenylacrylamide (6a) $600 \mathrm{MHz}, \mathrm{CDCl}_{3}, 23{ }^{\circ} \mathrm{C}$

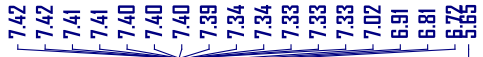

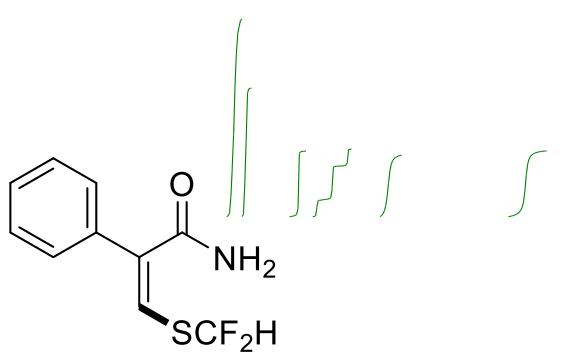

$6 a$

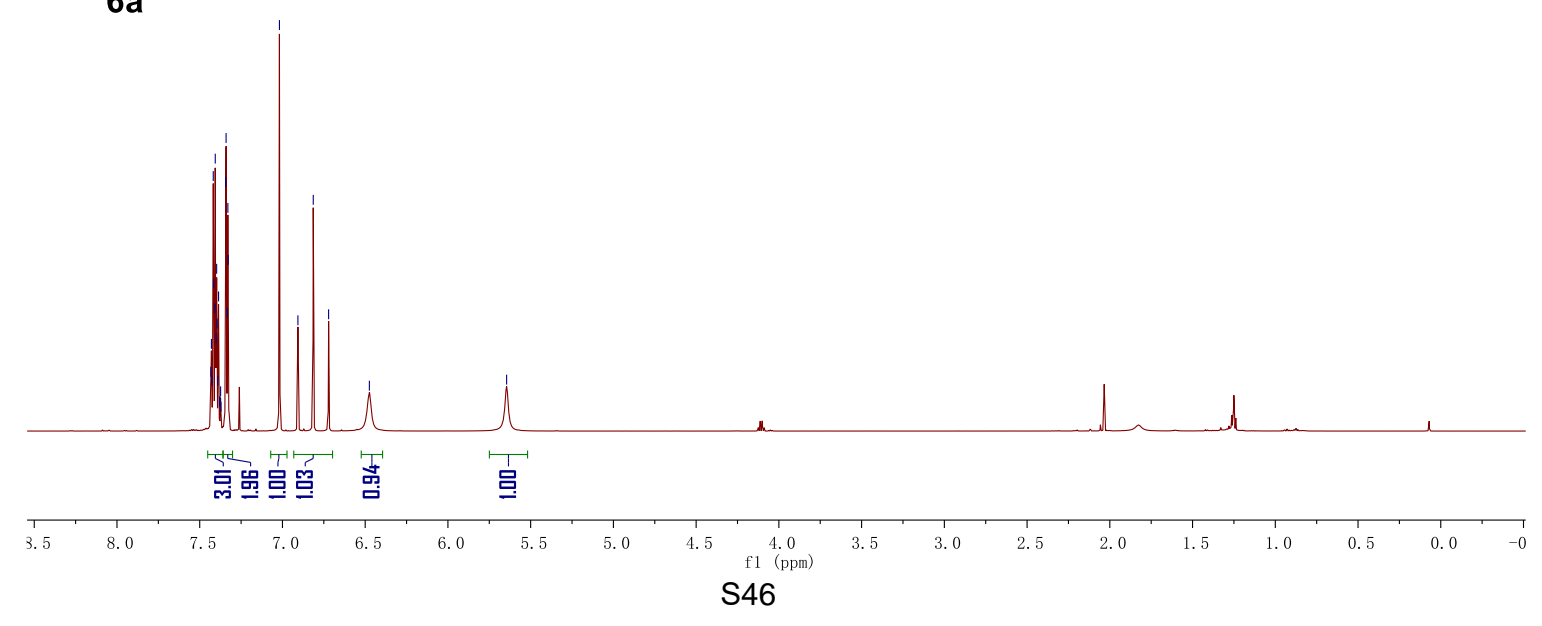


${ }^{13} \mathrm{C}$ NMR spectrum of (Z)-3-((difluoromethyl)thio)-2-phenylacrylamide (6a)

$151 \mathrm{MHz}, \mathrm{CDCl}_{3}, 23{ }^{\circ} \mathrm{C}$

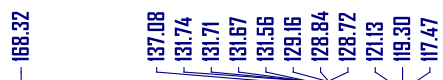

$\underbrace{\mathrm{N}}_{\mathrm{SCF}_{2} \mathrm{H}}$

$6 a$

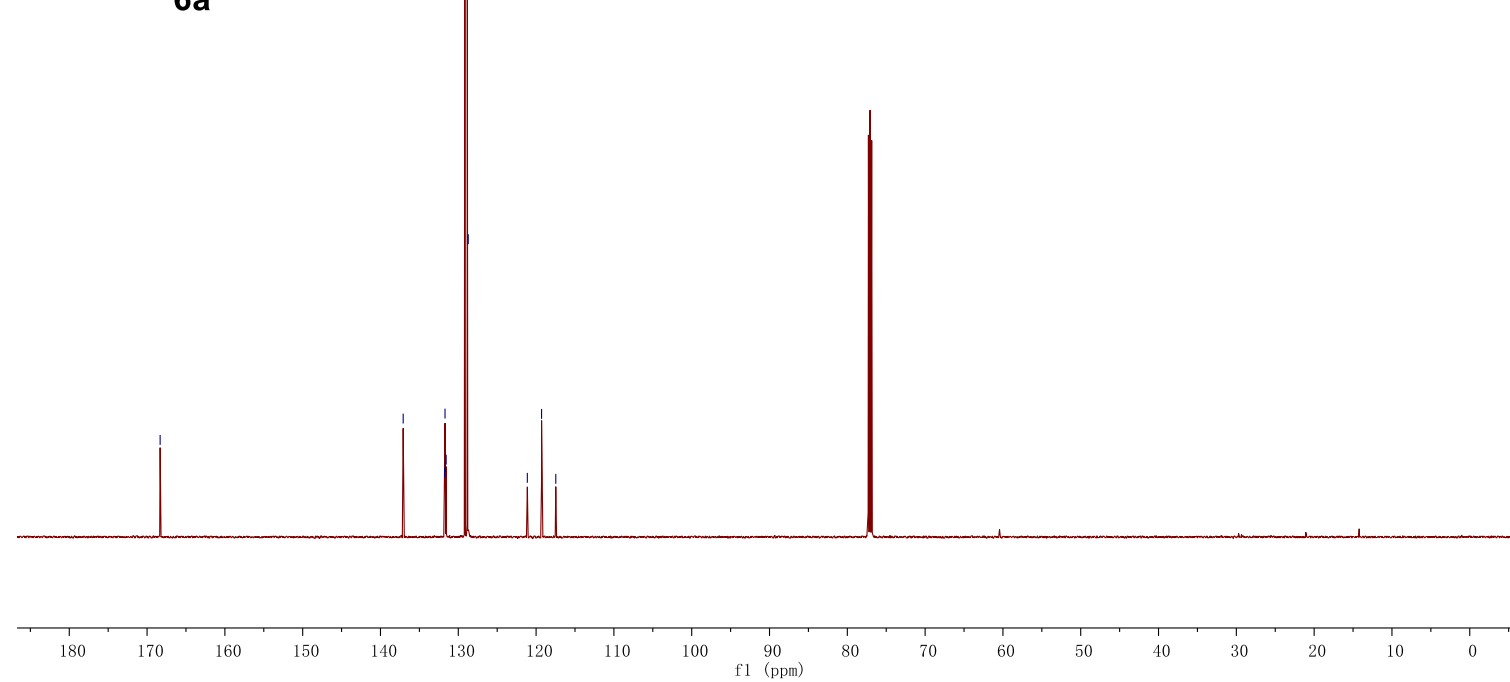

${ }^{19}$ F NMR spectrum of (Z)-3-((difluoromethyl)thio)-2-phenylacrylamide (6a)

$565 \mathrm{MHz}, \mathrm{CDCl}_{3}, 23{ }^{\circ} \mathrm{C}$<smiles>NC(=O)/C(=C\C(=O)F)c1ccccc1</smiles>

寄昏

$6 a$

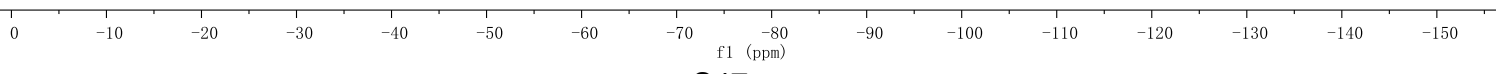

